\title{
Merging Halogen-Atom Transfer (XAT) with Copper Catalysis for the Modular Suzuki-Miyaura-Type Cross-Coupling of Alkyl Iodides and Organoborons
}

\author{
Zhenhua Zhang, ${ }^{1 \dagger}$ Bartosz Górski, ${ }^{1 \dagger}$ and Daniele Leonori ${ }^{1 *}$ \\ ${ }^{1}$ Department of Chemistry, University of Manchester, Oxford Road, Manchester M13 9PL, \\ $U K$. \\ *daniele.leonori@manchester.ac.uk \\ ${ }^{\dagger}$ These authors contributed equally to this work.
}

\section{Table of Contents}

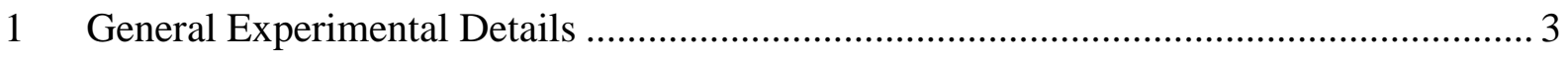

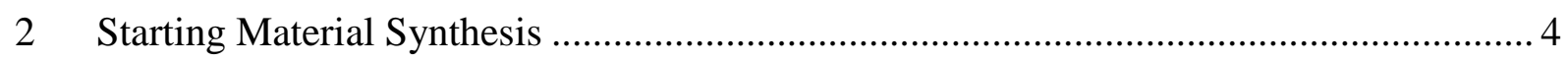

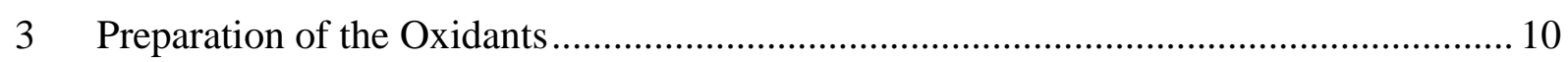

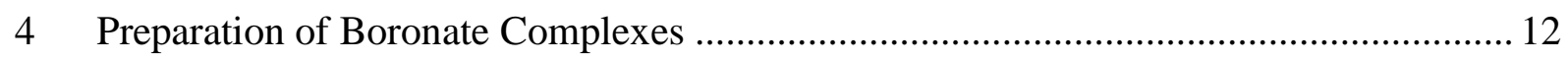

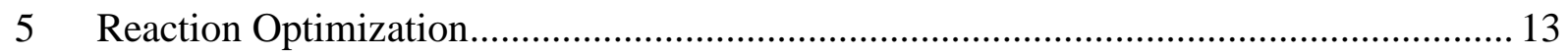

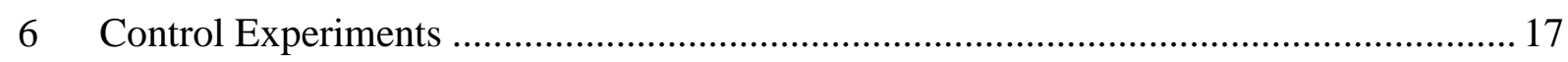

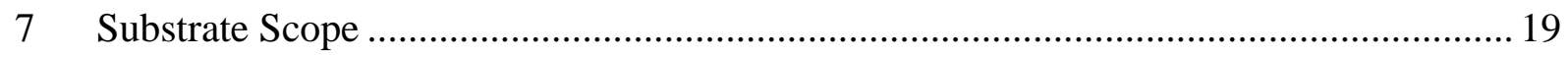

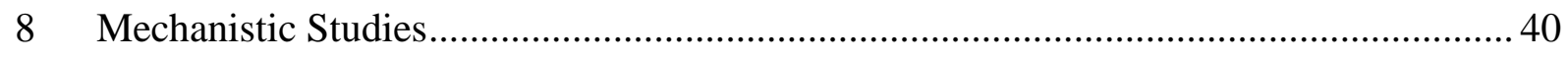

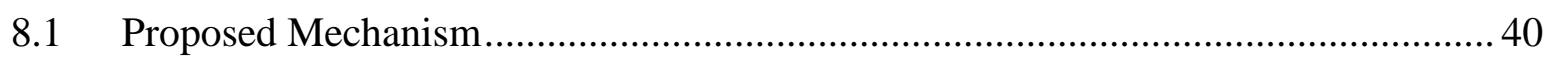

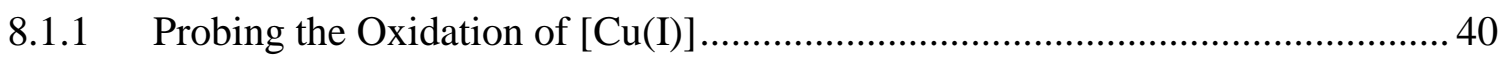

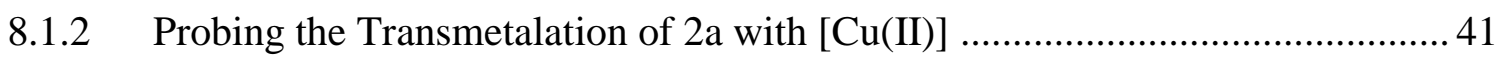

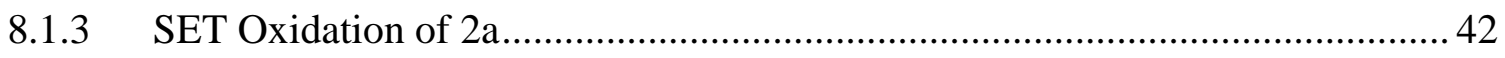

8.1.4 Probing $\alpha$-Aminoalkyl Radical Generation by HAT .................................... 43

8.1.5 Probing $\alpha$-Aminoalkyl Radical-Mediated XAT …....................................... 44

8.1.6 Ruling Out Direct SET Reduction of the Alkyl Iodide ................................. 45

8.1.7 Probing Radical Capture by $[\mathrm{Cu}(\mathrm{II})]-\mathrm{Ar}$ Species as $\mathrm{C}\left(s p^{3}\right)-\mathrm{C}\left(s p^{2}\right)$ Bond

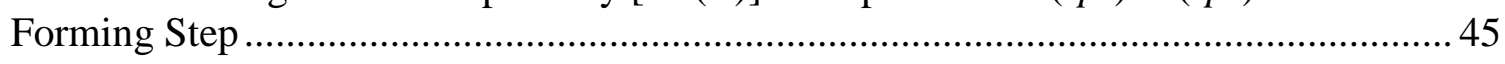

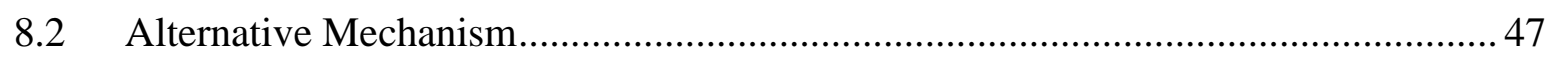

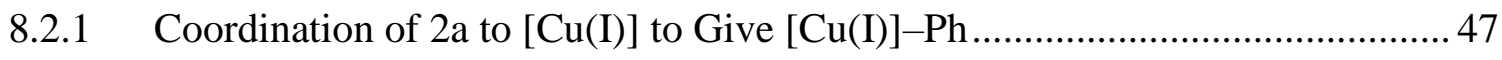




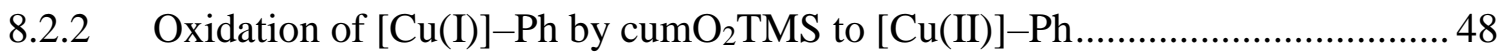

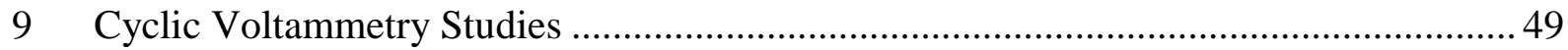

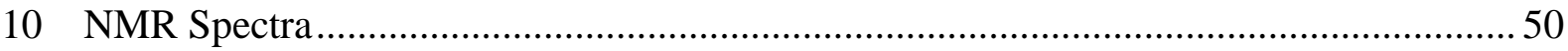

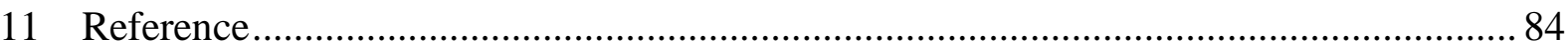




\section{General Experimental Details}

All required fine chemicals were used directly without purification unless stated otherwise. All air and moisture sensitive reactions were carried out under nitrogen atmosphere using standard Schlenk manifold technique. All solvents were bought from Acros as $99.8 \%$ purity. $1 \mathrm{H}$ and $13 \mathrm{C}$ Nuclear Magnetic Resonance (NMR) spectra were acquired at various field strengths as indicated and were referenced to $\mathrm{CHCl}_{3}\left(7.26\right.$ and $77.0 \mathrm{ppm}$ for ${ }^{1} \mathrm{H}$ and ${ }^{13} \mathrm{C}$ respectively). $1 \mathrm{H}$ NMR coupling constants are reported in Hertz and refer to apparent multiplicities and not true coupling constants. Data are reported as follows: chemical shift, integration, multiplicity ( $\mathrm{s}=$ singlet, br $\mathrm{s}=$ broad singlet, $\mathrm{d}=$ doublet, $\mathrm{t}=$ triplet, $\mathrm{q}=$ quartet, $\mathrm{p}=$ pentet, $\mathrm{h}=$ hextet, hept $=$ heptet, $\mathrm{m}=$ multiplet, $\mathrm{dd}=$ doublet of doublets, etc.), proton assignment (determined by ${ }^{2} \mathrm{D}$ NMR experiments: COSY, HSQC and HMBC) where possible. High-resolution mass spectra were obtained using a JEOL JMS-700 spectrometer or a Fissions VG Trio 2000 quadrupole mass spectrometer. Spectra were obtained using electron impact ionization (EI) and chemical ionization (CI) techniques, or positive electrospray (ES). Analytical TLC: aluminum backed plates pre-coated $(0.25 \mathrm{~mm})$ with Merck Silica Gel 60 F254. Compounds were visualized by exposure to UV-light or by dipping the plates in permanganate $(\mathrm{KMnO} 4)$ stain followed by heating. Flash column chromatography was performed using Merck Silica Gel 60 (40-63 $\mu \mathrm{m})$. All mixed solvent eluents are reported as v/v solutions. UV-visible absorption spectra were obtained using a Horiba Duetta spectrometer and $1 \mathrm{~mm}$ High Precision Cell made of quartz from Hellma Analytics $(c=5 \mathrm{mM})$. All the reactions were conducted in CEM $10 \mathrm{~mL}$ glass microwave tubes.

Some abbreviations used:

cumOOTMS: trimethylsilyl(cumyl) peroxide

cumOOTES: triethylsilyl(cumyl) peroxide

$\operatorname{cumOOSiPh}_{2} t \mathrm{Bu}: t$-butyldiphenyl(cumyl) peroxide

TMHD: 2,2,6,6-tetramethyl-3,5-heptanedione

RuPhos: 2-dicyclohexylphosphino-2',6'-diisopropoxybiphenyl

BINOL: 1,1'-bi(2-naphthol)

TMEDA: $N, N, N^{\prime}, N^{\prime}$-tetramethyl ethylenediamine

Xantophos: 4,5-bis(diphenylphosphino)-9,9-dimethylxanthene

dtbbpy: 4,4'-di-t-butyl-2,2'-dipyridyl

CuTc: copper(I) thiophene-2-carboxylate

CPME: cyclopentyl methyl ether 


\section{Starting Material Synthesis}

\section{General Procedure of the Appel Reaction - GP1}

$$
\begin{aligned}
& \mathrm{I}_{2} \text { (1.2 equiv.) } \\
& \mathrm{PPh}_{3} \text { (1.2 equiv.) }
\end{aligned}
$$

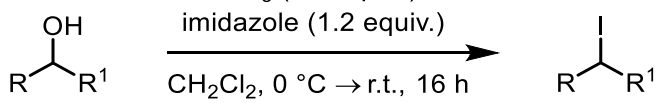

A round-bottom flask equipped with a stirring bar was charged with the corresponding alcohol (1.0 equiv.), $\mathrm{Ph}_{3} \mathrm{P}$ (1.2 equiv.) and imidazole (1.2 equiv.). The flask was evacuated and refilled with $\mathrm{N}_{2} . \mathrm{CH}_{2} \mathrm{Cl}_{2}(0.1 \mathrm{M})$ was added, and the reaction was cooled to $0{ }^{\circ} \mathrm{C}$ with an ice-water bath. $\mathrm{I}_{2}$ (1.2 equiv.) was added portion-wise and then the cooling bath was removed. The reaction was stirred 16 hours at room temperature and then diluted with $\mathrm{H}_{2} \mathrm{O}(30 \mathrm{~mL})$. The layers were separated and the aqueous layer was extracted with $\mathrm{CH}_{2} \mathrm{Cl}_{2}(3 \times 30 \mathrm{~mL})$. The combined organic layers were washed with $\mathrm{Na}_{2} \mathrm{~S}_{2} \mathrm{O}_{3}\left(30 \mathrm{~mL}\right.$, saturated), brine $(30 \mathrm{~mL})$, dried $\left(\mathrm{MgSO}_{4}\right)$, filtered and evaporated. Purification by flash column chromatography on silica gel gave the products.

\section{Diisopropyl 3-Iodocyclobutane-1,1-dicarboxylate (S1)}

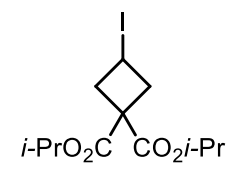

Following GP1, diisopropyl 3-hydroxycyclobutane-1,1-dicarboxylate (1.22 g, $5.0 \mathrm{mmol})$ gave S1 $(1.45 \mathrm{mg}, 82 \%)$ as an oil. ${ }^{1} \mathrm{H}$ NMR $\left(400 \mathrm{MHz}, \mathrm{CDCl}_{3}\right) \delta 5.07(1 \mathrm{H}$, hept, $J=6.3 \mathrm{~Hz}), 5.06$ (1H, hept, $J=6.3 \mathrm{~Hz}), 4.52(1 \mathrm{H}, \mathrm{p}, J=8.4 \mathrm{~Hz}), 3.24-3.11(2 \mathrm{H}, \mathrm{m}), 3.08-2.95(2 \mathrm{H}, \mathrm{m}), 1.24$ $(6 \mathrm{H}, \mathrm{d}, J=2.1 \mathrm{~Hz}), 1.23(6 \mathrm{H}, \mathrm{d}, J=2.1 \mathrm{~Hz}) ;{ }^{13} \mathrm{C} \mathrm{NMR}\left(101 \mathrm{MHz}, \mathrm{CDCl}_{3}\right) \delta 170.4,169.0,69.5$, 69.3, 54.2, 42.1, 21.5, 6.7; HRMS (ASAP): Found $\mathrm{MH}^{+} 355.0398, \mathrm{C}_{12} \mathrm{H}_{20} \mathrm{O}_{4} \mathrm{I}$ requires 355.0398 .

\section{(2-Iodopropyl)benzene (S2)}

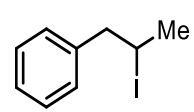

Following GP1, 1-phenylpropan-2-ol (1.36 g, $10.0 \mathrm{mmol})$ gave $\mathbf{S 2}$ as an oil (1.19 g, 48\%). ${ }^{1} \mathrm{H}$ NMR $\left(400 \mathrm{MHz}, \mathrm{CDCl}_{3}\right) \delta$ 7.32-7.24 $(3 \mathrm{H}, \mathrm{m}), 7.17(2 \mathrm{H}, \mathrm{d}, J=6.5 \mathrm{~Hz}), 4.33(1 \mathrm{H}, \mathrm{h}, J=6.8$ $\mathrm{Hz}), 3.31-3.26(1 \mathrm{H}, \mathrm{m}), 3.08-3.03(1 \mathrm{H}, \mathrm{m}), 1.89(3 \mathrm{H}, \mathrm{d}, J=6.5 \mathrm{~Hz})$. Data in accordance with the literature. ${ }^{1}$ 
8-Iodo-1,4-dioxaspiro[4.5]decane (S3)

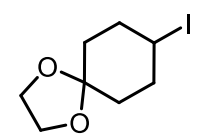

Following GP1 1,4-dioxaspiro[4.5]decan-8-ol (0.79 g, $5.0 \mathrm{mmol})$ gave $\mathbf{S 3}$ as an oil (0.99 g, 74\%). ${ }^{1} \mathrm{H}$ NMR (400 MHz, $\left.\mathrm{CDCl}_{3}\right) \delta 4.49-4.32(1 \mathrm{H}, \mathrm{m}), 4.01-3.83(4 \mathrm{H}, \mathrm{m}), 2.15-2.02(4 \mathrm{H}$, m), $1.80(2 \mathrm{H}, \mathrm{ddd}, J=12.4,7.7,4.9 \mathrm{~Hz}), 1.60(2 \mathrm{H}, \mathrm{ddd}, J=13.1,8.3,4.6 \mathrm{~Hz})$. Data in accordance with the literature. ${ }^{2}$

\section{4-Iodotetrahydro-2H-thiopyran (S4)}

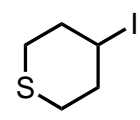

Following GP1, tetrahydrothiopyran-4-ol (0.96 g, $8.2 \mathrm{mmol})$ gave $\mathbf{S 4}$ as an oil $(1.41 \mathrm{~g}, 75 \%)$. ${ }^{1} \mathrm{H}$ NMR (400 MHz, $\left.\mathrm{CDCl}_{3}\right) \delta 4.55-4.40(1 \mathrm{H}, \mathrm{m}), 2.90-2.74(2 \mathrm{H}, \mathrm{m}), 2.65-2.45(2 \mathrm{H}, \mathrm{m}), 2.40-$ $2.15(4 \mathrm{H}, \mathrm{m})$. Data in accordance with the literature. ${ }^{3}$

\section{tert-Butyl 6-Iodo-2-azaspiro[3.3]heptane-2-carboxylate (S5)}

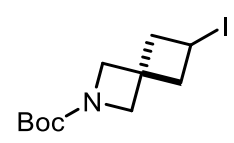

Following GP1, tert-butyl 6-hydroxy-2-azaspiro[3.3] heptane-2-carboxylate (0.85 g, $4.0 \mathrm{mmol})$ gave $\mathbf{S 5}$ as a solid $(0.97 \mathrm{~g}, 75 \%) .{ }^{1} \mathrm{H}$ NMR $\left(400 \mathrm{MHz}, \mathrm{CDCl}_{3}\right) \delta 4.29(1 \mathrm{H}, \mathrm{p}, J=7.8 \mathrm{~Hz}), 3.94$ $(4 \mathrm{H}, \mathrm{d}, J=12.3 \mathrm{~Hz}), 2.96-2.87(2 \mathrm{H}, \mathrm{m}), 2.74-2.66(2 \mathrm{H}, \mathrm{m}), 1.42(9 \mathrm{H}, \mathrm{s})$. Data in accordance with the literature. ${ }^{4}$

tert-Butyl 2-Iodo-7-azaspiro[3.5]nonane-7-carboxylate (S6)

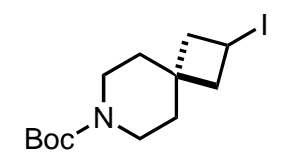

Following GP1, but using toluene as a solvent, reflux, $16 \mathrm{~h}$, tert-butyl 2-hydroxy-7azaspiro[3.5]nonane-7-carboxylate $(0.96 \mathrm{~g}, 4.0 \mathrm{mmol})$ gave $\mathbf{S 6}$ as a solid $(0.96 \mathrm{~g}, 68 \%) .{ }^{1} \mathrm{H}$ NMR (400 MHz, $\left.\mathrm{CDCl}_{3}\right) \delta 4.49(1 \mathrm{H}, \mathrm{p}, J=8.3 \mathrm{~Hz}), 3.35-3.29(2 \mathrm{H}, \mathrm{m}), 3.29-3.25(2 \mathrm{H}, \mathrm{m})$, 2.70-2.61 (2H, m), 2.46-2.38 (2H, m), 1.72-1.63 (2H, m), 1.59-1.52 (2H, m), 1.44 (9H, s). Data in accordance with the literature. ${ }^{4}$ 
tert-Butyl 3-exo-Iodo-8-azabicyclo[3.2.1]octane-8-carboxylate (S7)

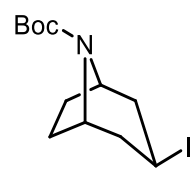

Following GP1 tert-butyl endo-3-hydroxy-8-azabicyclo[3.2.1]octane-8-carboxylate (1.56 g, $6.93 \mathrm{mmol})$ gave $\mathbf{S} 7$ as a solid $(1.69 \mathrm{~g}, 73 \%) .{ }^{1} \mathrm{H} \mathrm{NMR}\left(400 \mathrm{MHz}, \mathrm{CDCl}_{3}\right) \delta 4.51(1 \mathrm{H}, \mathrm{tt}, J=$ 11.9, $5.7 \mathrm{H}), 4.14-4.04(1 \mathrm{H}, \mathrm{m}), 4.04-3.92(1 \mathrm{H}, \mathrm{m}), 2.47-2.23(2 \mathrm{H}, \mathrm{m}), 2.22-2.14(2 \mathrm{H}, \mathrm{m})$, $1.98-1.83(2 \mathrm{H}, \mathrm{m}), 1.71-1.57(2 \mathrm{H}, \mathrm{m}), 1.47(9 \mathrm{H}, \mathrm{s}) ;{ }^{13} \mathrm{C} \mathrm{NMR}$ (101 MHz, $\mathrm{CDCl}_{3}$, rotamers) $\delta$ $150.2,81.2,56.2,55.6,45.3,44.5,28.5,27.7,27.1,18.5$. Data in accordance to the literature. ${ }^{5}$

2-Iodo-2,3-dihydro-1H-indene (S8)

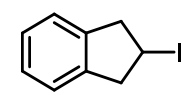

Following GP1 2,3-dihydro-1H-inden-2-ol (1.34 g, $10.0 \mathrm{mmol})$ gave S8 as a solid (2.19 g, 90\%). ${ }^{1} \mathrm{H}$ NMR $\left(500 \mathrm{MHz}, \mathrm{CDCl}_{3}\right) \delta 7.38-7.10(4 \mathrm{H}, \mathrm{m}), 4.76(1 \mathrm{H}, \mathrm{tt}, J=6.2,5.0 \mathrm{~Hz}), 3.51$ $(2 \mathrm{H}, \mathrm{dd}, J=16.8,6.5 \mathrm{~Hz}), 3.34(2 \mathrm{H}, \mathrm{dd}, J=16.8,5.0 \mathrm{~Hz})$. Data in accordance with the literature. $^{6}$

\section{4-Iodocyclohexan-1-one (S9)}

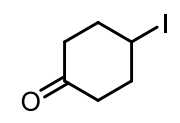

Following GP1 4-hydroxycyclohexan-1-one (570 mg, $5.0 \mathrm{mmol})$ gave $\mathbf{S 9}$ as a solid (871 $\mathrm{mg}$, 78\%). ${ }^{1} \mathrm{H}$ NMR (400 MHz, $\left.\mathrm{CDCl}_{3}\right) \delta$ 4.73-4.68 (1H, m), 2.68-2.63 (2H, m), 2.40-2.25 (4H, $\mathrm{m}), 2.20-2.15(2 \mathrm{H}, \mathrm{m})$. Data in accordance with the literature. ${ }^{7}$

\section{2-(4-Iodopiperidin-1-yl)pyrimidine (S10)}

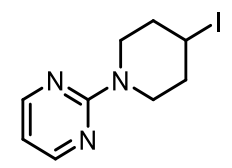

Following GP1 1-(pyrimidin-2-yl)piperidin-4-ol (3.56 g, $20.0 \mathrm{mmol}$ ) gave S10 as a solid (5.19 g, 90\%). ${ }^{1} \mathrm{H}$ NMR (400 MHz, $\left.\mathrm{CDCl}_{3}\right) \delta 8.30(2 \mathrm{H}, \mathrm{d}, J=4.7 \mathrm{~Hz}), 6.48(1 \mathrm{H}, \mathrm{t}, J=4.7 \mathrm{~Hz}), 4.55$ $(1 \mathrm{H}, \mathrm{tt}, \mathrm{J}=6.9,5.1 \mathrm{~Hz}), 4.13-4.04(2 \mathrm{H}, \mathrm{m}), 3.63(2 \mathrm{H}, \mathrm{ddd}, \mathrm{J}=13.7,6.9,4.7 \mathrm{~Hz}), 2.17-2.07(4 \mathrm{H}$, $\mathrm{m})$. Data in accordance with the literature. ${ }^{2}$ 


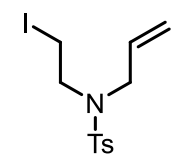

Following GP1 $N$-allyl- $N$-(2-hydroxyethyl)-4-methylbenzenesulfonamide (2.55 g, 10.0 mmol) gave $\mathbf{S 1 1}$ as a solid (3.00 g, 82\%). ${ }^{1} \mathrm{H}$ NMR (400 MHz, $\left.\mathrm{CDCl}_{3}\right) \delta 7.69(2 \mathrm{H}, \mathrm{d}, J=8.0 \mathrm{~Hz}), 7.31$ $(2 \mathrm{H}, \mathrm{d}, J=8.0 \mathrm{~Hz}), 5.70-5.63(1 \mathrm{H}, \mathrm{m}), 5.18(2 \mathrm{H}, \mathrm{t}, J=8.8 \mathrm{~Hz}), 3.78(2 \mathrm{H}, \mathrm{d}, J=6.4 \mathrm{~Hz}), 3.41$ $(2 \mathrm{H}, \mathrm{t}, J=7.7 \mathrm{~Hz}), 3.22(2 \mathrm{H}, \mathrm{t}, J=8.5 \mathrm{~Hz}), 2.43(3 \mathrm{H}, \mathrm{s})$. Data in accordance with the literature. ${ }^{8}$

\section{7-(2-Iodopropyl)-1,3-dimethyl-3,7-dihydro-1H-purine-2,6-dione (S12)}

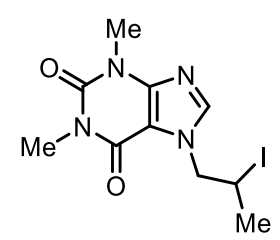

Following GP1, 7-(2-hydroxypropyl)-1,3-dimethyl-3,7-dihydro-1H-purine-2,6-dione (1.00 g, $4.2 \mathrm{mmol}$ ) gave $\mathbf{S 1 2}$ as a solid (1.08 g, 74\%). ${ }^{1} \mathrm{H}$ NMR (500 MHz, CDCl3) $\delta 7.65$ (1H, s), 4.61$4.54(1 \mathrm{H}, \mathrm{m}), 4.50(1 \mathrm{H}, \mathrm{dd}, J=14.1,5.0 \mathrm{~Hz}), 4.37(1 \mathrm{H}, \mathrm{dd}, J=14.1,9.0 \mathrm{~Hz}), 3.60(3 \mathrm{H}, \mathrm{s}), 3.40$ $(3 \mathrm{H}, \mathrm{s}), 1.96(3 \mathrm{H}, \mathrm{d}, J=6.9 \mathrm{~Hz}) ;{ }^{13} \mathrm{C} \mathrm{NMR}(126 \mathrm{MHz}, \mathrm{CDCl} 3) \delta 155.4,151.7,149.4$, 141.6, 106.7, 56.8, 30.0, 28.2, 25.4, 25.0. Data in accordance with the literature. ${ }^{9}$

\section{trans-1-Ethoxy-2-iodocyclohexane (S13)}

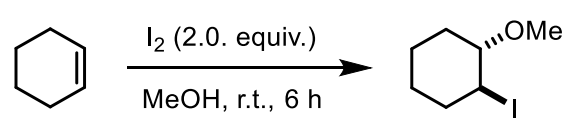

A round-bottom flask equipped with a stirring bar was charged with cyclohexene (1.23 g, 15.0 mmol, 1.0 equiv.) and $\mathrm{MeOH}(45 \mathrm{~mL}, 0.3 \mathrm{M})$. $\mathrm{I}_{2}$ (7.61 g, $30.0 \mathrm{mmol}, 2.0$ equiv.) was slowly added to the vigorously stirred solution. After $6 \mathrm{~h}$, the reaction mixture was diluted with $\mathrm{Et}_{2} \mathrm{O}$ $(50 \mathrm{~mL})$ and $\mathrm{Na}_{2} \mathrm{~S}_{2} \mathrm{O}_{3}(30 \mathrm{~mL}$, sat.). The layers were separated and the organic layer was washed with $\mathrm{H}_{2} \mathrm{O}(2 \times 30 \mathrm{~mL})$, brine $(30 \mathrm{~mL})$ and dried $\left(\mathrm{MgSO}_{4}\right)$. The residue was then evaporated and purified by distillation to afford $\mathbf{S 1 3}$ as an oil (2.85 g, 79\%). ${ }^{1} \mathrm{H} \mathrm{NMR}\left(500 \mathrm{MHz}, \mathrm{CDCl}_{3}\right) \delta 4.05$ $(1 \mathrm{H}, \mathrm{ddd}, J=10.7,8.7,4.2 \mathrm{~Hz}), 3.39(3 \mathrm{H}, \mathrm{s}), 3.22(1 \mathrm{H}, \mathrm{td}, J=9.0,4.1 \mathrm{~Hz}), 2.41-2.33(1 \mathrm{H}, \mathrm{m})$, $2.21-2.14(1 \mathrm{H}, \mathrm{m}), 2.02-1.89(1 \mathrm{H}, \mathrm{m}), 1.80(1 \mathrm{H}, \mathrm{dtt}, J=11.6,4.5,1.8 \mathrm{~Hz}), 1.52(1 \mathrm{H}, \mathrm{ddtt}, J=$ $12.0,5.1,3.4,1.8 \mathrm{~Hz}), 1.41-1.16(3 \mathrm{H}, \mathrm{m})$. Data in accordance with the literature. ${ }^{10}$ 
trans-2-(Allyloxy)-3-iodotetrahydro-2H-pyran (S14)

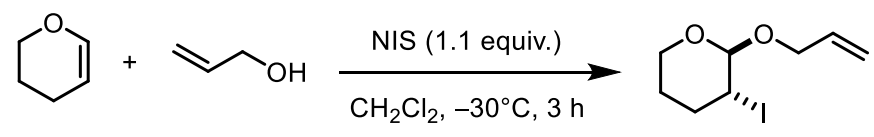

A round-bottom flask equipped with a stirring bar was charged with 3,4-dihydro- $2 \mathrm{H}$-pyran (315 mg, 3.75 mmol, 1.25 equiv.), allyl alcohol (174 mg, $3.0 \mathrm{mmol}, 1.0$ equiv.) and $\mathrm{CH}_{2} \mathrm{Cl}_{2}(6 \mathrm{~mL}$, $0.5 \mathrm{M}$ ). The reaction was cooled to $-30{ }^{\circ} \mathrm{C}$ and then $\mathrm{N}$-iodosuccinimide $(742 \mathrm{~g}, 3.3 \mathrm{mmol}, 1.1$ equiv.) was slowly added to the vigorously stirred solution. After $3 \mathrm{~h}$, the mixture was diluted with $\mathrm{Et}_{2} \mathrm{O}(50 \mathrm{~mL})$ and $\mathrm{Na}_{2} \mathrm{~S}_{2} \mathrm{O}_{3}(30 \mathrm{~mL}$, sat.). The layers were separated and the organic layer was washed with $\mathrm{H}_{2} \mathrm{O}(2 \times 30 \mathrm{~mL})$, brine $(30 \mathrm{~mL})$, dried $\left(\mathrm{MgSO}_{4}\right)$ and evaporated. Purification by flash column chromatography on silica gel eluting hexane-EtOAc, gave $\mathbf{S 1 4}$ as an oil (435 $\mathrm{mg}, 54 \%) .{ }^{1} \mathrm{H}$ NMR (400 MHz, $\left.\mathrm{CDCl}_{3}\right) \delta 6.00-5.86(1 \mathrm{H}, \mathrm{m}), 5.32(1 \mathrm{H}, \mathrm{dq}, J=17.3,1.7 \mathrm{~Hz})$, $5.32(1 \mathrm{H}, \mathrm{dq}, J=17.3,1.7 \mathrm{~Hz}), 5.20(1 \mathrm{H}, \mathrm{dq}, J=10.4,1.4 \mathrm{~Hz}), 4.67(1 \mathrm{H}, \mathrm{d}, J=5.3 \mathrm{~Hz}), 4.25$ (1H, ddt, $J=12.9,5.2,1.5 \mathrm{~Hz}), 4.16-3.90(3 \mathrm{H}, \mathrm{m}), 3.58$ (1H, ddd, $J=11.2,7.4,3.5 \mathrm{~Hz}), 2.46-$ $2.28(1 \mathrm{H}, \mathrm{m}),, 2.10-1.95(1 \mathrm{H}, \mathrm{m}), 1.85-1.71(1 \mathrm{H}, \mathrm{m}), 1.66-1.50(1 \mathrm{H}, \mathrm{m})$. Data in accordance with the literature. ${ }^{11}$

\section{4-Iodo-1-oxaspiro[5.5] undecane (S15)}

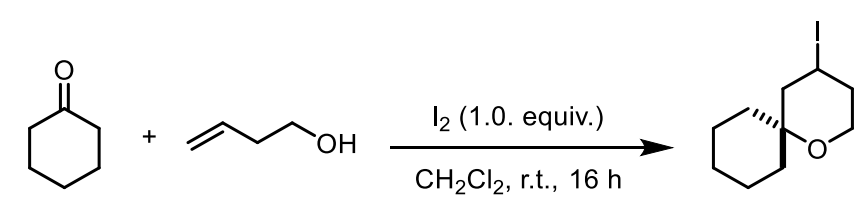

A round-bottom flask equipped with a stirring bar was charged with cyclohexanone $(981 \mathrm{mg}$, 10.0 mmol, 1.0 equiv.), but-3-en-1-ol (1.44 g, 20.0 mmol, 2.0 equiv.) and $\mathrm{CH}_{2} \mathrm{Cl}_{2}$ (50 mL, 0.2 M). $\mathrm{I}_{2}$ (2.53 g, $10.0 \mathrm{mmol}, 1.0$ equiv.) was slowly added to the vigorously stirred solution. After $6 \mathrm{~h}$, the mixture was diluted with $\mathrm{Et}_{2} \mathrm{O}(50 \mathrm{~mL})$ and $\mathrm{Na}_{2} \mathrm{~S}_{2} \mathrm{O}_{3}(30 \mathrm{~mL}$, sat.). The layers were separated and the organic layer was washed with $\mathrm{H}_{2} \mathrm{O}(2 \times 30 \mathrm{~mL})$, brine $(30 \mathrm{~mL})$, dried $\left(\mathrm{MgSO}_{4}\right)$ and evaporated. Purification by flash column chromatography on silica gel eluting hexane-EtOAc, gave $\mathbf{S 1 5}$ as an oil $(1.17 \mathrm{~g}, 42 \%) .{ }^{1} \mathrm{H}$ NMR $\left(500 \mathrm{MHz}, \mathrm{CDCl}_{3}\right) \delta 4.48(1 \mathrm{H}$, tt, $J=12.5,4.4 \mathrm{~Hz}), 3.67-3.54(2 \mathrm{H}, \mathrm{m}), 2.34-2.15$ (2H, m), 2.06-1.92 (2H, m), 1.69-1.19 (10H, $\mathrm{m})$. Data in accordance with the literature. ${ }^{12}$ 


\section{2-(4-Bromophenyl)-4-iodotetrahydro-2H-pyran (S16)}

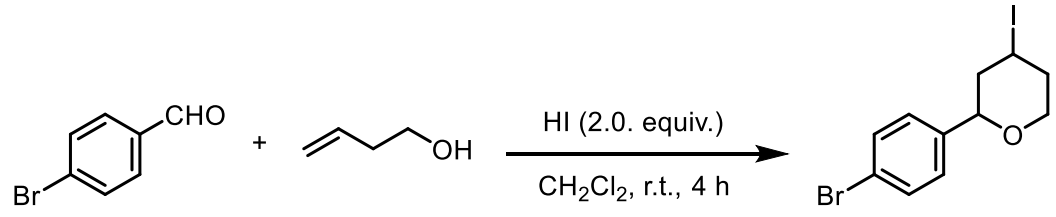

A round bottomed flask equipped with a stirring bar was charged with 4-bromobenzaldehyde (0.3 g, $1.6 \mathrm{mmol}, 1.0$ equiv.), $\mathrm{CH}_{2} \mathrm{Cl}_{2}$ (16 mL), but-3-en-1-ol (0.28 mL, $3.2 \mathrm{mmol}, 2.0$ equiv.) and $\mathrm{HI}(0.4 \mathrm{~mL}, 55 \mathrm{wt} \%$ solution in water, $3.2 \mathrm{mmol}, 2.0$ equiv.). The mixture was stirred at room temperature for $4 \mathrm{~h}$ when it was judged complete (TLC analysis). The mixture was diluted with $\mathrm{H}_{2} \mathrm{O}(30 \mathrm{~mL})$, the layers were separated, and the aqueous layer was extracted with $\mathrm{CH}_{2} \mathrm{Cl}_{2}$ ( $3 \times 30 \mathrm{~mL}$ ). The combined organic layers were washed with $\mathrm{Na}_{2} \mathrm{~S}_{2} \mathrm{O}_{3}$ (30 mL, $10 \%$ solution), brine $(30 \mathrm{~mL})$, dried $\left(\mathrm{MgSO}_{4}\right)$, filtered, and evaporated. Purification by flash column chromatography on silica gel eluting hexane-EtOAc, gave $\mathbf{S 1 6}(0.29 \mathrm{~g}, 49 \%)$ as a solid. ${ }^{1} \mathrm{H}$ $\operatorname{NMR}\left(400 \mathrm{MHz}, \mathrm{CDCl}_{3}\right) \delta 7.46(2 \mathrm{H}, \mathrm{d}, J=8.5 \mathrm{~Hz}), 7.22(2 \mathrm{H}, \mathrm{d}, J=8.5 \mathrm{~Hz}), 4.91(1 \mathrm{H}, \mathrm{t}, J=$ $3.3 \mathrm{~Hz}), 4.80(1 \mathrm{H}, \mathrm{dd}, J=10.6,2.1 \mathrm{~Hz}), 4.05(2 \mathrm{H}, \mathrm{t}, J=5.6 \mathrm{~Hz}), 2.18(1 \mathrm{H}, \mathrm{dt}, J=14.7,2.7$ $\mathrm{Hz}), 1.96(2 \mathrm{H}, \mathrm{dt}, J=5.3,2.7 \mathrm{~Hz}), 1.81(1 \mathrm{H}, \mathrm{ddd}, J=14.5,10.6,3.5 \mathrm{~Hz})$. Data in accordance with the literature. ${ }^{13}$ 


\section{Preparation of the Oxidants}

\section{Preparation of cumOOTMS}

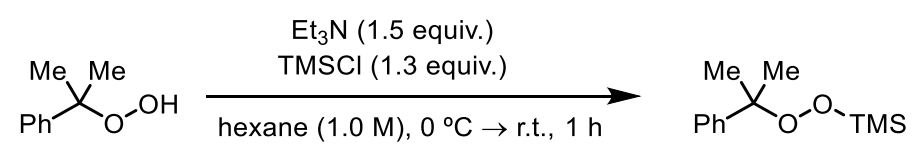

A round bottom flask equipped with a stirring bar was charged with $\mathrm{Et}_{3} \mathrm{~N}(10.5 \mathrm{~mL}, 75.0 \mathrm{mmol})$ and hexane $(100 \mathrm{~mL})$. Cumene hydroperoxide $(9.3 \mathrm{~mL}, 80 \%$ technical solution, $50.0 \mathrm{mmol})$ was slowly added. The reaction media was then cooled in an ice-water bath and TMSCl $(8.25$ $\mathrm{mL}, 65.0 \mathrm{mmol}$ ) was slowly added. When the addition was completed, the ice-water bath was removed and the reaction was let stirring at room temperature. After 1 hour, $\mathrm{H}_{2} \mathrm{O}(100 \mathrm{~mL})$ was added. The layers were separated and the organic phase was washed with $\mathrm{H}_{2} \mathrm{O}(100 \mathrm{~mL} \times 4)$ and brine $(100 \mathrm{~mL})$, dried $\left(\mathrm{MgSO}_{4}\right)$, filtered and evaporated to give cumOOTMS as an oil (10.97 g, 98\%) with approx density of $0.94 \mathrm{~g} / \mathrm{mL} .{ }^{1} \mathrm{H}$ NMR $\left(400 \mathrm{MHz}, \mathrm{CDCl}_{3}\right) \delta 7.45(2 \mathrm{H}, \mathrm{d}$, $J=8.4 \mathrm{~Hz}), 7.33(2 \mathrm{H}, \mathrm{t}, J=7.7 \mathrm{~Hz}), 7.24(1 \mathrm{H}, \mathrm{t}, J=7.9 \mathrm{~Hz}), 1.58(6 \mathrm{H}, \mathrm{s}), 0.18(9 \mathrm{H}, \mathrm{s}) ;{ }^{13} \mathrm{C}$ NMR $\left(101 \mathrm{MHz}, \mathrm{CDCl}_{3}\right) \delta 145.4,127.9,127.0,125.6,83.4,26.5,-1.1$. Data in accordance with the literature. ${ }^{5}$

\section{Preparation of cumOOTES}

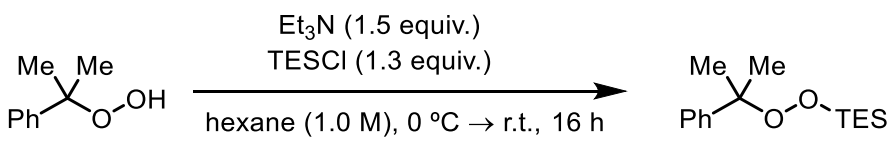

A round bottom flask equipped with a stirring bar was charged with $\mathrm{Et}_{3} \mathrm{~N}(10.5 \mathrm{~mL}, 75.0 \mathrm{mmol})$ and hexane $(100 \mathrm{~mL})$. Cumene hydroperoxide $(9.3 \mathrm{~mL}, 80 \%$ technical solution, $50.0 \mathrm{mmol})$ was slowly added. The reaction media was then cooled in an ice-water bath and TESCl (10.9 $\mathrm{mL}, 65.0 \mathrm{mmol}$ ) was slowly added. When the addition was completed, the ice-water bath was removed and the reaction was let stirring at room temperature. After 16 hours, $\mathrm{H}_{2} \mathrm{O}(100 \mathrm{~mL})$ was added. The layers were separated and the organic phase was washed with $\mathrm{H}_{2} \mathrm{O}$ (100 $\mathrm{mL} x$ 4) and brine $(100 \mathrm{~mL})$, dried $\left(\mathrm{MgSO}_{4}\right)$, filtered through a short pad of $\mathrm{SiO}_{2}$ and evaporated to give cumOOTES as an oil $(10.42 \mathrm{~g}, 78 \%)$ with approx density of $0.94 \mathrm{~g} / \mathrm{mL} .{ }^{1} \mathrm{H}$ NMR (400 MHz, $\left.\mathrm{CDCl}_{3}\right)$ ઈ 7.48-7.42 (2H, m), 7.37-7.30 (2H, m), 7.27-7.21 (1H, m), 1.57 $(6 \mathrm{H}, \mathrm{s}), 0.98(9 \mathrm{H}, \mathrm{t}, J=7.9 \mathrm{~Hz}), 0.69(6 \mathrm{H}, \mathrm{q}, J=8.0 \mathrm{~Hz}) ;{ }^{13} \mathrm{C} \mathrm{NMR}\left(101 \mathrm{MHz}, \mathrm{CDCl}_{3}\right) \delta 145.8$, $127.9,126.8,125.5,83.3,26.5,6.8,3.9$. Data in accordance with the literature. ${ }^{14}$ 


\section{Preparation of cumOOSiPh $2 t-B u(S 17)$}

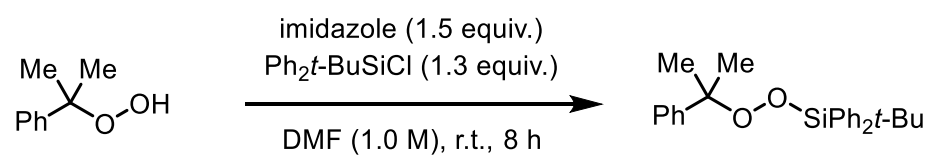

A round bottom flask equipped with a stirring bar was charged with imidazole $(1.0 \mathrm{~g}, 15 \mathrm{mmol}$, 1.5 equiv.), cumene hydroperoxide ( $1.85 \mathrm{~mL}, 80 \%$ technical solution, $10.0 \mathrm{mmol}, 1.00$ equiv.) and DMF (10 mL). $\mathrm{Ph}_{2} t$-BuSiCl $(3.3 \mathrm{~mL}, 13 \mathrm{mmol}, 1.3$ equiv.) was slowly added at room temperature. After 8 hours, $\mathrm{H}_{2} \mathrm{O}(80 \mathrm{~mL})$ and $\mathrm{Et}_{2} \mathrm{O}(120 \mathrm{~mL})$ were added. The layers were separated and the organic phase was washed with $\mathrm{H}_{2} \mathrm{O}(80 \mathrm{~mL} \times 4)$ and brine $(100 \mathrm{~mL})$, dried $\left(\mathrm{MgSO}_{4}\right)$. Solvent was removed under reduced pressure and the residue was purified by flash column chromatography on silica gel eluting hexane-EtOAc to give cumOOSiPh $2 t-\mathrm{Bu} \mathbf{S 1 7}$ as an oil (1.6 g, 40\%). ${ }^{1} \mathrm{H}$ NMR (400 MHz, $\left.\mathrm{CDCl}_{3}\right) \delta 8.01-7.91(4 \mathrm{H}, \mathrm{m}), 7.59(8 \mathrm{H}, \mathrm{m}), 7.51-7.38$ $(3 \mathrm{H}, \mathrm{m}), 1.77$ (6H, s), $1.35(9 \mathrm{H}, \mathrm{s}) ;{ }^{13} \mathrm{C} \mathrm{NMR}\left(101 \mathrm{MHz}, \mathrm{CDCl}_{3}\right) \delta 145.5,136.1,133.3,129.9$, 128.0, 127.6, 127.0, 125.8, 84.5, 27.5, 26.6, 19.7; HRMS (ESI) found: $\mathrm{MNa}^{+}$413.1896, $\mathrm{C}_{25} \mathrm{H}_{30} \mathrm{O}_{2} \mathrm{SiNa}$ requires 413.1907 .

\section{Stability studies of cumOOTMS}

CumOOTMS does not have explosion propagating property and can be used on scale as we have discussed previously. ${ }^{5}$ 


\section{Preparation of Boronate Complexes}

\section{General Procedure for the Boronate Preparation - GP2}

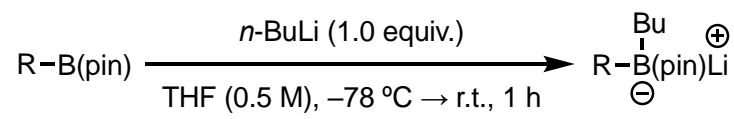

An oven-dry tube equipped with a stirring bar was charged with the boronic ester (1.1 equiv.). The tube was capped with a Supelco aluminium crimp seal with septum (PTFE/butyl), evacuated and refilled with $\mathrm{N}_{2}$ (x 3). Dry and degassed THF (0.5 M) was added and the tube was cooled to $-78{ }^{\circ} \mathrm{C}$. $n$-BuLi (1.0 equiv., $2.5 \mathrm{M}$ in hexanes) was added dropwise and after 1 minute the cooling bath was removed and the stirring was continued for $1 \mathrm{~h}$. The THF solution of the boronate was directly used in the coupling reactions.

The structures of all boronates prepared are shown in Scheme S1. All boronic acid pinacol esters used for the preparation of boronates complexes are commercially available.

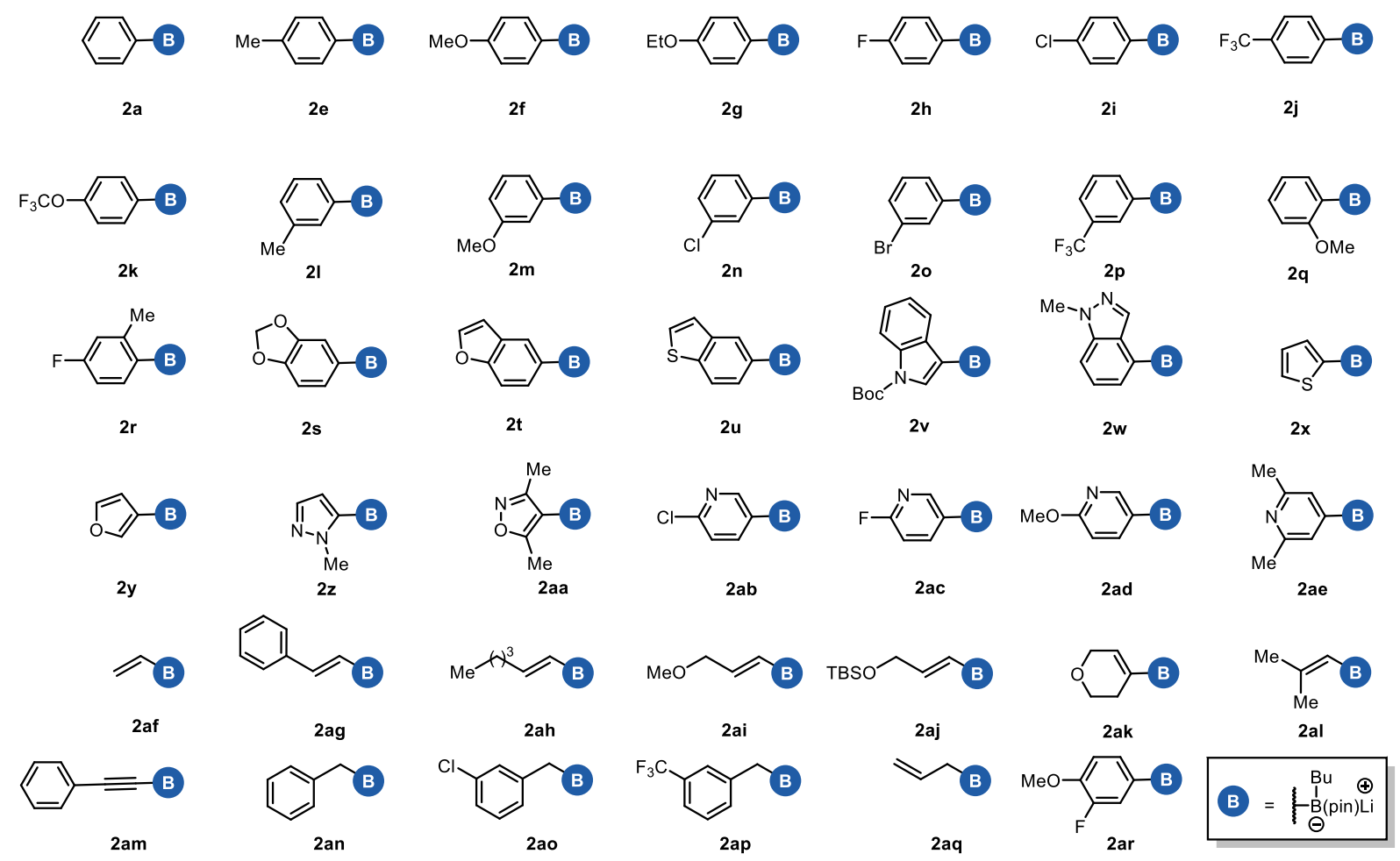

Scheme S1. 


\section{$5 \quad$ Reaction Optimization}

\section{General Procedure for the Reaction Optimization - GP3}

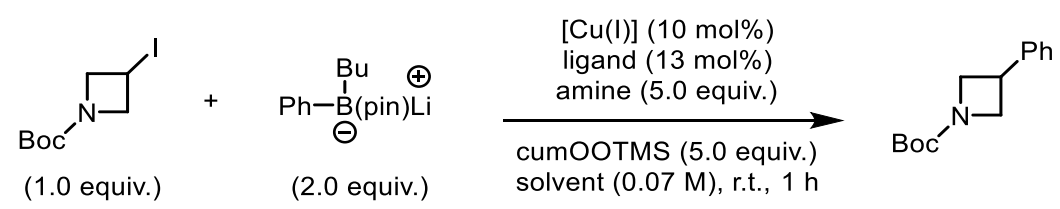

An oven-dry tube equipped with a stirring bar was charged with the $[\mathrm{Cu}(\mathrm{I})]$ catalyst $(0.010$ $\mathrm{mmol}, 10 \mathrm{~mol} \%)$ and the ligand $(0.013 \mathrm{mmol}, 13 \mathrm{~mol} \%)$. The tube was capped with a Supelco aluminium crimp seal with septum (PTFE/butyl), evacuated and refilled with $\mathrm{N}_{2}$ (x 3). Dry and degassed solvents (1.0 mL), tert-butyl 3-iodoazetidine-1-carboxylate (28 mg, $0.10 \mathrm{mmol}, 1.0$ equiv.), the amine ( $0.50 \mathrm{mmol}, 5.0$ equiv.), boronate $2 \mathrm{a}(0.50 \mathrm{~mL}, 0.5 \mathrm{M}$ in THF, $0.25 \mathrm{mmol}$, 2.5 equiv. $)$ were sequentially addled. Finally, the oxidant $(0.50 \mathrm{mmol}, 5.0$ equiv. $)$ was added dropwise keeping the reaction mixture under vigorous stirring. After $1 \mathrm{~h}, \mathrm{NH}_{4} \mathrm{Cl}$ sat. $(1 \mathrm{~mL})$ was added and the mixture was stirred for $3 \mathrm{~min}$. EtOAc $(20 \mathrm{~mL})$ and a solution of 1,3dinitrobenzene (internal standard) in EtOAc ( $1 \mathrm{~mL}, 0.05 \mathrm{M}, 0.5$ equiv.) were added. The mixture was washed with water $(20 \mathrm{~mL})$, brine $(20 \mathrm{~mL})$, dried $\left(\mathrm{MgSO}_{4}\right)$, filtered, and evaporated. The yield was determined by ${ }^{1} \mathrm{H}$ NMR using 1,3-dinitrobenzene as the internal standard. 
Table S1.

\begin{tabular}{|c|c|c|c|c|c|}
\hline Entry & {$[\mathrm{Cu}(\mathrm{I})]$} & Ligand & Amine & Solvent & Yield $(\%)$ \\
\hline 1 & \multirow{11}{*}[\mathrm{Cu}(\mathrm{CH}_{3}\mathrm{CN})_{4}]{$\mathrm{PF}_{6}$} & TMHD & \multirow{16}{*}{$\mathrm{Et}_{3} \mathrm{~N}$} & \multirow{16}{*}{ DMSO } & 55 \\
\hline 2 & & RuPhos & & & 25 \\
\hline 3 & & TMEDA & & & 21 \\
\hline 4 & & Xantphos & & & 40 \\
\hline 5 & & 1,10-phenanthroline & & & 20 \\
\hline 6 & & BINOL & & & 29 \\
\hline 7 & & 2-pyridinecarboxylic acid & & & 41 \\
\hline 8 & & terpyridine & & & 50 \\
\hline 10 & & 1,3-diphenyl-1,3-propanedione & & & 26 \\
\hline 11 & & biquinoline & & & 30 \\
\hline 12 & & dtbbpy & & & 14 \\
\hline 13 & $\mathrm{CuI}$ & \multirow{5}{*}{ TMHD } & & & 39 \\
\hline 14 & CuTC & & & & 44 \\
\hline 15 & $(\mathrm{CuOTf})_{2} \mathrm{PhCH}_{3}$ & & & & 33 \\
\hline 16 & $\mathrm{CuCN}$ & & & & 33 \\
\hline 17 & $\mathrm{CuOAc}$ & & & & 12 \\
\hline
\end{tabular}




\begin{tabular}{|c|c|c|c|c|c|}
\hline Entry & {$[\mathrm{Cu}]$} & Ligand & Amine & Solvent & Yield (\%) \\
\hline 18 & \multirow{13}{*}[\mathrm{Cu}(\mathrm{CH}_{3}\mathrm{CN})_{4}]{$\mathrm{PF}_{6}$} & \multirow{13}{*}{ TMHD } & $n-\mathrm{Bu}_{3} \mathrm{~N}$ & \multirow{4}{*}{ DMSO } & 31 \\
\hline 19 & & & $i-\mathrm{Pr}_{3} \mathrm{~N}$ & & 47 \\
\hline 20 & & & PMP & & 42 \\
\hline 21 & & & $i$ - $\mathrm{Pr}_{2} \mathrm{NMe}$ & & 38 \\
\hline 22 & & & \multirow{9}{*}{$\mathrm{Et}_{3} \mathrm{~N}$} & NMP & 13 \\
\hline 23 & & & & DMA & 20 \\
\hline 24 & & & & DMPU & 5 \\
\hline 25 & & & & $\mathrm{CH}_{3} \mathrm{CN}$ & 17 \\
\hline 26 & & & & EtOAc & 0 \\
\hline 27 & & & & THF & 0 \\
\hline 28 & & & & dioxane & 0 \\
\hline 29 & & & & glyme & 0 \\
\hline 30 & & & & CPME & 0 \\
\hline
\end{tabular}

Since no significant further improvements were obtained we focused on evaluating the ratio of reagents used in Table S1 entry 1 (Table S2). 


\section{Table S2.}

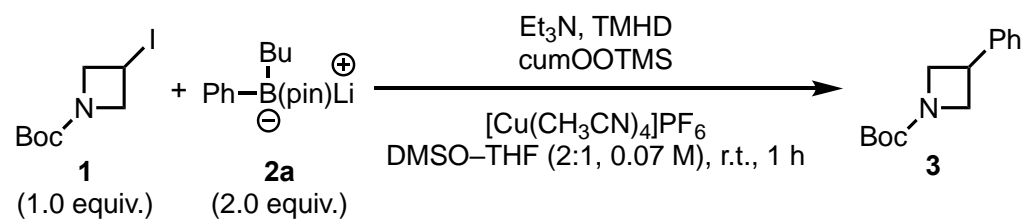

\begin{tabular}{|c|c|c|c|c|c|c|}
\hline Entry & 2a (equiv.) & Et3 $_{3} \mathbf{N}$ (equiv.) & cumOOTMS (equiv.) & TMHD (mol\%) & {$\left[\mathrm{Cu}\left(\mathrm{CH}_{3} \mathrm{CN}\right)_{4}\right] \mathrm{PF}_{6}\left(\mathrm{~mol}^{2}\right)$} & Yield (\%) \\
\hline 1 & 2.0 & 5 & 5 & 13 & 10 & 55 \\
\hline 2 & 2.0 & 5 & 4 & 13 & 10 & 54 \\
\hline 3 & 2.0 & 5 & 3 & 13 & 10 & 58 \\
\hline 4 & 2.0 & 5 & 2 & 13 & 10 & 59 \\
\hline 5 & 2.0 & 5 & 1.5 & 13 & 10 & 40 \\
\hline 6 & 2.0 & 4 & 2 & 13 & 10 & 60 \\
\hline 7 & 2.0 & 3 & 2 & 13 & 10 & 56 \\
\hline 8 & 2.0 & 2 & 2 & 13 & 10 & 57 \\
\hline 9 & 2.0 & 2 & 3 & 13 & 10 & 62 \\
\hline 10 & 2.0 & 2 & 4 & 13 & 10 & 55 \\
\hline 11 & 2.0 & 2 & 5 & 13 & 10 & 50 \\
\hline 12 & 2.0 & 3 & 3 & 13 & 10 & 64 \\
\hline 13 & 2.5 & 3 & 3 & 25 & 20 & 72 \\
\hline
\end{tabular}




\section{Control Experiments}

Control experiments were set up based on the optimised reaction conditions (Scheme S2).

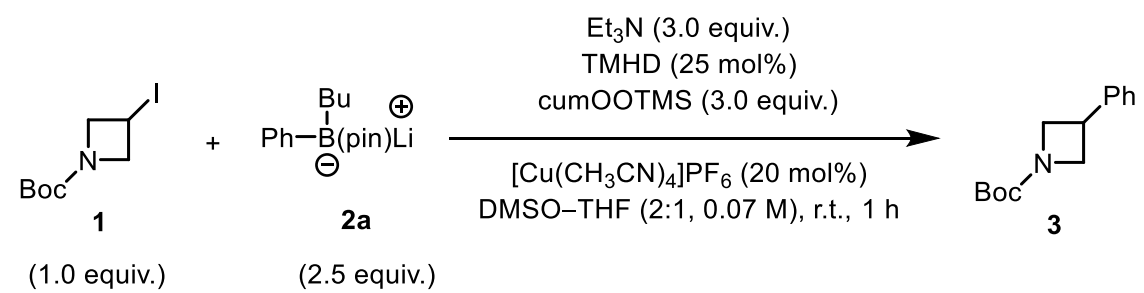

Scheme S2.

Table S3.

\begin{tabular}{|c|c|c|c|}
\hline Entry & Deviation from the reaction conditions & Yield (\%) & $\mathbf{1}(\boldsymbol{\%})$ \\
\hline $\mathbf{1}$ & none & 72 & - \\
\hline $\mathbf{2}$ & no Et $3 \mathrm{~N}$ & 16 & 80 \\
\hline $\mathbf{3}$ & no $\left[\mathrm{Cu}\left(\mathrm{CH}_{3} \mathrm{CN}\right)_{4}\right] \mathrm{PF}_{6}$ & - & 100 \\
\hline $\mathbf{4}$ & no TMHD & 37 & - \\
\hline $\mathbf{5}$ & no cumOOTMS & - & 100 \\
\hline
\end{tabular}

Reaction in the absence of $\mathrm{Et}_{3} \mathrm{~N}$ provided 3, but in lower yield (Table S3, entry 3). Upon analysis of the reaction crude by GC-MS we identified the formation of $\mathrm{Ph}-\mathrm{I}$ and acetophenone (Scheme S3). We believe that 2a might eventually be oxidised by cumOOTMS to form either a $\mathrm{Ph} \bullet$ or a $\mathrm{Bu} \bullet$, both of which can activate $\mathbf{1}$ by XAT and thus lead to product formation. However, $\mathrm{Bu}-\mathrm{I}$ was not detected in the reaction mixture.

An alternative mechanistic pathway that could lead to product formation in the presence of the amine, would involve the fragmentation of the cumO• to form Me that has been demonstrated as a XAT mediator. Furthermore, we have been able to detect benzophenone in the crude mixture. 

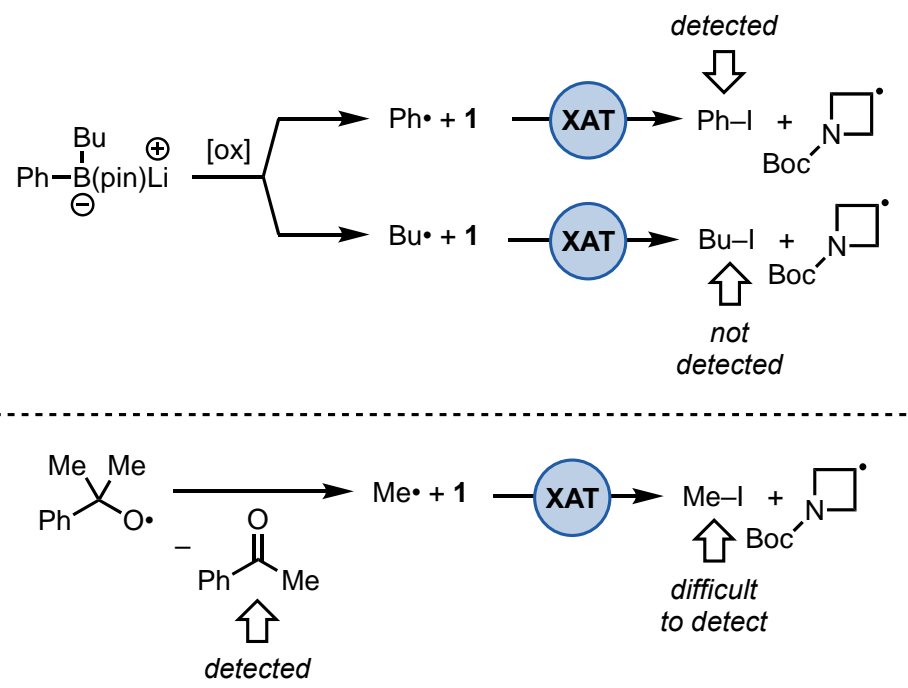

Scheme S3. 


\section{Substrate Scope}

\section{General Procedure for the C-C Coupling Reaction - GP4}

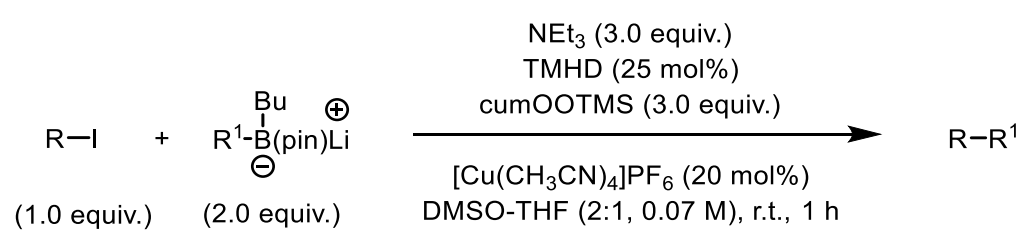

An oven-dry tube equipped with a stirring bar was charged with the alkyl iodide $(0.10 \mathrm{mmol}$, 1.0 equiv) and $\left[\mathrm{Cu}(\mathrm{MeCN})_{4}\right] \mathrm{PF}_{6}(7.5 \mathrm{mg}, 0.020 \mathrm{mmol}, 20 \mathrm{~mol} \%)$. The tube was capped with a Supelco aluminium crimp seal with septum (PTFE/butyl), evacuated and refilled with $\mathrm{N}_{2}$ (x 3). Dry and degassed DMSO (1.0 mL), TMHD (5.2 $\mu \mathrm{L}, 0.025 \mathrm{mmol}, 25 \mathrm{~mol} \%), \mathrm{NEt}_{3}(42 \mu \mathrm{L}, 0.30$ mmol, 3.0 equiv.) and the boronate $(0.5 \mathrm{~mL}, 0.25 \mathrm{mmol}, 2.5$ equiv., $0.5 \mathrm{M}$ in THF) were sequentially added. cumOOTMS (67 mg, $72 \mu \mathrm{L}, 0.30 \mathrm{mmol}, 3.0$ equiv.) was added dropwise to the vigorously stirred solution. Stirring was continued for $1 \mathrm{~h}$, then $\mathrm{NH}_{4} \mathrm{Cl}$ sat. $(20 \mathrm{~mL})$, EtOAc $(20 \mathrm{~mL})$ were added. The organic layer was separated and the aqueous layer was extracted with EtOAc (15 mL x 2). The combined organic phases were washed with water (20 $\mathrm{mL})$, brine $(20 \mathrm{~mL})$, dried $\left(\mathrm{MgSO}_{4}\right)$, filtered, and evaporated. The crude mixture was purified by flash column chromatography on silica gel.

\section{tert-Butyl 3-Phenylazetidine-1-carboxylate (3)}

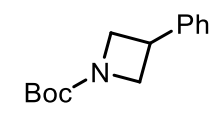

Following GP4, 1-Boc-3-iodoazetidine $(19 \mu \mathrm{L}, 0.10 \mathrm{mmol})$ and boronate $2 \mathbf{a}(0.50 \mathrm{~mL}, 0.50 \mathrm{M}$ in THF, $0.25 \mathrm{mmol})$ gave $3(17 \mathrm{mg}, 72 \%)$ as an oil. ${ }^{1} \mathrm{H}$ NMR (400 $\left.\mathrm{MHz}, \mathrm{CDCl}_{3}\right) \delta 7.36-7.19$ $(5 \mathrm{H}, \mathrm{m}), 4.31(2 \mathrm{H}, \mathrm{t}, J=8.7 \mathrm{~Hz}), 3.97(2 \mathrm{H}, \mathrm{dd}, J=8.6,6.1 \mathrm{~Hz}), 3.71(1 \mathrm{H}, \mathrm{m}), 1.46(9 \mathrm{H}, \mathrm{s})$. Data in accordance with the literature. ${ }^{15}$

\section{tert-Butyl 3-(p-Tolyl)azetidine-1-carboxylate (6)}

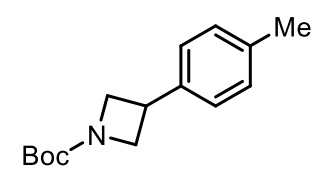

Following GP4, 1-Boc-3-iodoazetidine $(19 \mu \mathrm{L}, 0.10 \mathrm{mmol})$, boronate $2 \mathrm{e}(0.50 \mathrm{~mL}, 0.50 \mathrm{M}$ in

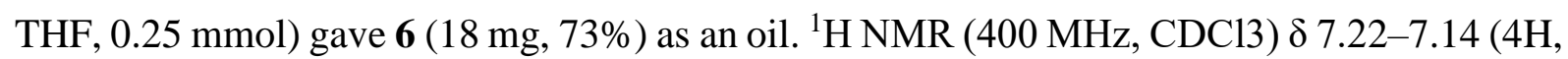
m), $4.31(2 \mathrm{H}, \mathrm{t}, J=8.7 \mathrm{~Hz}), 3.98-3.94(2 \mathrm{H}, \mathrm{m}), 3.73-3.67(1 \mathrm{H}, \mathrm{m}), 2.34(3 \mathrm{H}, \mathrm{s}), 1.47(9 \mathrm{H}, \mathrm{s})$. Data in accordance with the literature. ${ }^{16}$ 
tert-Butyl 3-(4-Methoxyphenyl)azetidine-1-carboxylate (7)

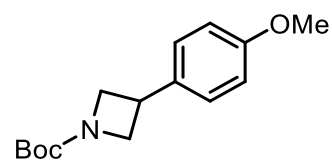

Following GP4, 1-Boc-3-iodoazetidine $(19 \mu \mathrm{L}, 0.10 \mathrm{mmol})$, boronate $\mathbf{2 f}(0.50 \mathrm{~mL}, 0.50 \mathrm{M}$ in THF, $0.25 \mathrm{mmol})$ gave 7 (21 mg, 78\%) as an oil. ${ }^{1} \mathrm{H} \mathrm{NMR}\left(400 \mathrm{MHz}, \mathrm{CDCl}_{3}\right) \delta 7.24(2 \mathrm{H}$, dd, $J=8.5,2.1 \mathrm{~Hz}), 6.88(2 \mathrm{H}, \mathrm{d}, J=8.6 \mathrm{~Hz}), 4.30(2 \mathrm{H}, \mathrm{t}, J=8.6 \mathrm{~Hz}), 3.93(2 \mathrm{H}, \mathrm{dd}, J=8.6,6.0$ $\mathrm{Hz}), 3.80(3 \mathrm{H}, \mathrm{s}), 3.72-3.62(1 \mathrm{H}, \mathrm{m}), 1.46(9 \mathrm{H}, \mathrm{s})$. Data in accordance with the literature. ${ }^{17}$

tert-Butyl 3-(4-Ethoxyphenyl)azetidine-1-carboxylate (8)<smiles>CCOc1ccc(C2CN(C(=O)OC(C)(C)C)C2)cc1</smiles>

Following GP4, 1-Boc-3-iodoazetidine $(19 \mu \mathrm{L}, 0.10 \mathrm{mmol})$, boronate $2 \mathrm{~g}(0.50 \mathrm{~mL}, 0.50 \mathrm{M}$ in THF, $0.25 \mathrm{mmol}$ ) gave 8 (22 mg, 81\%) as an oil. ${ }^{1} \mathrm{H}$ NMR (400 MHz, $\left.\mathrm{CDCl}_{3}\right) \delta 7.24-7.18(2 \mathrm{H}$, m), 6.90-6.85 (2H, m), $4.30(2 \mathrm{H}, \mathrm{t}, J=8.6 \mathrm{~Hz}), 4.03(2 \mathrm{H}, \mathrm{q}, J=7.0 \mathrm{~Hz}), 3.93(2 \mathrm{H}, \mathrm{dd}, J=8.5$, $6.1 \mathrm{~Hz}), 3.67(1 \mathrm{H}, \mathrm{tt}, J=8.7,6.1 \mathrm{~Hz}), 1.46(9 \mathrm{H}, \mathrm{s}), 1.41(3 \mathrm{H}, \mathrm{t}, J=7.0 \mathrm{~Hz}) ;{ }^{13} \mathrm{C}$ NMR $(101$ $\left.\mathrm{MHz}_{\mathrm{CDCl}}\right) \delta 158.1,156.6,134.3,127.9,114.8,79.6,63.6,56.7,33.0,28.6,15.0 ;$ HRMS (ESI) found: $\mathrm{MNa}^{+} 300.1570, \mathrm{C}_{16} \mathrm{H}_{23} \mathrm{O}_{3} \mathrm{NNa}$ requires 300.1570 .

tert-Butyl 3-(4-Fluorophenyl)azetidine-1-carboxylate (9)

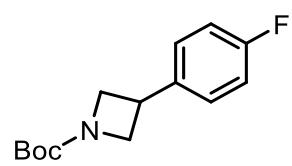

Following GP4, 1-Boc-3-iodoazetidine $(19 \mu \mathrm{L}, 0.10 \mathrm{mmol})$, boronate $\mathbf{2 h}(0.50 \mathrm{~mL}, 0.50 \mathrm{M}$ in THF, $0.25 \mathrm{mmol})$ gave $9(16 \mathrm{mg}, 63 \%)$ as an oil. ${ }^{1} \mathrm{H} \mathrm{NMR}\left(400 \mathrm{MHz}, \mathrm{CDCl}_{3}\right) \delta 7.32-7.26(2 \mathrm{H}$, m), 7.07-7.01 (2H, m), $4.32(2 \mathrm{H}, \mathrm{t}, J=8.7 \mathrm{~Hz}), 3.96-3.90(2 \mathrm{H}, \mathrm{m}), 3.74-3.63(1 \mathrm{H}, \mathrm{m}), 1.47$ $(9 \mathrm{H}, \mathrm{s})$. Data in accordance with the literature. ${ }^{16}$

tert-Butyl 3-(4-Chlorophenyl)azetidine-1-carboxylate (10)

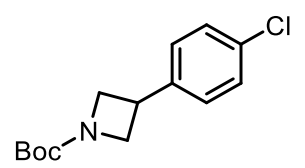

Following GP4, 1-Boc-3-iodoazetidine $(19 \mu \mathrm{L}, 0.10 \mathrm{mmol})$, boronate $2 \mathbf{i}(0.50 \mathrm{~mL}, 0.50 \mathrm{M}$ in THF, $0.25 \mathrm{mmol})$ gave 10 (16 mg, 58\%) as an oil. ${ }^{1} \mathrm{H} \mathrm{NMR}\left(400 \mathrm{MHz}, \mathrm{CDCl}_{3}\right) 7.30(2 \mathrm{H}, \mathrm{d}, J$ 
$=8.5 \mathrm{~Hz}), 7.23(2 \mathrm{H}, \mathrm{d}, J=8.5 \mathrm{~Hz}), 4.31(2 \mathrm{H}, \mathrm{t}, J=8.7 \mathrm{~Hz}), 3.92(2 \mathrm{H}, \mathrm{dd}, J=8.7,6.0 \mathrm{~Hz})$, $3.73-3.65(1 \mathrm{H}, \mathrm{m}), 1.45(9 \mathrm{H}, \mathrm{s})$. Data in accordance with the literature. ${ }^{18}$

\section{tert-Butyl 3-(4-(Trifluoromethyl)phenyl)azetidine-1-carboxylate (11)}<smiles>O=C(c1ccccc1)N1CC(c2ccc(C(F)(F)F)cc2)C1</smiles>

Following GP4, 1-Boc-3-iodoazetidine ( $19 \mu \mathrm{L}, 0.10 \mathrm{mmol})$, boronate $2 \mathbf{j}(0.50 \mathrm{~mL}, 0.50 \mathrm{M}$ in THF, $0.25 \mathrm{mmol})$ gave $11(13 \mathrm{mg}, 44 \%)$ as an oil. ${ }^{1} \mathrm{H} \mathrm{NMR}\left(500 \mathrm{MHz}, \mathrm{CDCl}_{3}\right) \delta$ ppm: 7.60 $(2 \mathrm{H}, \mathrm{d}, J=8.0 \mathrm{~Hz}),, 7.43(2 \mathrm{H}, \mathrm{d}, J=8.1 \mathrm{~Hz}), 4.36(2 \mathrm{H}, \mathrm{t}, J=8.7 \mathrm{~Hz}), 3.97(2 \mathrm{H}, \mathrm{m}), 3.82-3.76$ $(1 \mathrm{H}, \mathrm{m}), 1.47(9 \mathrm{H}, \mathrm{s})$. Data in accordance with the literature. ${ }^{16}$

tert-Butyl 3-(4-(Trifluoromethoxy)phenyl)azetidine-1-carboxylate (12)<smiles>CCOc1ccc(C2CN(C(=O)c3ccccc3)C2)cc1</smiles>

Following GP4, 1-Boc-3-iodoazetidine $(19 \mu \mathrm{L}, 0.10 \mathrm{mmol})$, boronate $2 \mathbf{k}(0.50 \mathrm{~mL}, 0.50 \mathrm{M}$ in THF, $0.25 \mathrm{mmol}$ ) gave $12(19 \mathrm{mg}, 61 \%)$ as an oil. ${ }^{1} \mathrm{H} \mathrm{NMR}\left(400 \mathrm{MHz}, \mathrm{CDCl}_{3}\right) \delta 7.34-7.32$ $(2 \mathrm{H}, \mathrm{m}), 7.21-7.18(2 \mathrm{H}, \mathrm{m}), 4.34(2 \mathrm{H}, \mathrm{t}, J=8.7 \mathrm{~Hz}), 3.96-3.90(2 \mathrm{H}, \mathrm{m}), 3.77-3.68(1 \mathrm{H}, \mathrm{m})$, $1.47(9 \mathrm{H}, \mathrm{s})$. Data in accordance with the literature. ${ }^{18}$

tert-Butyl 3-(m-Tolyl)azetidine-1-carboxylate (13)<smiles>CC(C)(C)OC(=O)N1CC(c2cccc([N+](=O)[O-])c2)C1</smiles>

Following GP4, 1-Boc-3-iodoazetidine $(19 \mu \mathrm{L}, 0.10 \mathrm{mmol})$, boronate $2 \mathrm{l}(0.50 \mathrm{~mL}, 0.50 \mathrm{M}$ in THF, $0.25 \mathrm{mmol}$ ) gave $13(15 \mathrm{mg}, 60 \%)$ as an oil. ${ }^{1} \mathrm{H} \mathrm{NMR}\left(400 \mathrm{MHz}, \mathrm{CDCl}_{3}\right) \delta$ ppm: 7.25$7.21(1 \mathrm{H}, \mathrm{m}), 7.14-7.05(3 \mathrm{H}, \mathrm{m}), 4.31(2 \mathrm{H}, \mathrm{t}, J=8.6 \mathrm{~Hz}), 4.02-3.93(2 \mathrm{H}, \mathrm{m}), 3.74-3.68(1 \mathrm{H}$, $\mathrm{m}), 2.36(3 \mathrm{H}, \mathrm{s}), 1.47(9 \mathrm{H}, \mathrm{s})$. Data in accordance with the literature. ${ }^{16}$

tert-Butyl 3-(3-Methoxyphenyl)azetidine-1-carboxylate (14)

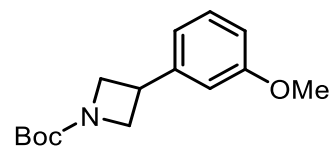

Following GP4, 1-Boc-3-iodoazetidine $(19 \mu \mathrm{L}, 0.10 \mathrm{mmol})$, boronate $2 \mathbf{m}(0.50 \mathrm{~mL}, 0.50 \mathrm{M}$ in THF, $0.25 \mathrm{mmol}$ ) gave 14 (19 mg, 74\%) as an oil. ${ }^{1} \mathrm{H} \mathrm{NMR}\left(400 \mathrm{MHz}, \mathrm{CDCl}_{3}\right) \delta$ ppm: $7.28-$ 
$7.23(1 \mathrm{H}, \mathrm{m}), 6.91-6.77(3 \mathrm{H}, \mathrm{m}), 4.31(2 \mathrm{H}, \mathrm{t}, J=8.6 \mathrm{~Hz}), 4.00-3.94(2 \mathrm{H}, \mathrm{m}), 3.81(3 \mathrm{H}, \mathrm{s})$, $3.75-3.66(1 \mathrm{H}, \mathrm{m}), 1.47(9 \mathrm{H}, \mathrm{s})$. Data in accordance with the literature. ${ }^{16}$

tert-Butyl 3-(3-Chlorophenyl)azetidine-1-carboxylate (15)<smiles>O=C(c1ccccc1)N1CC(c2cccc(Cl)c2)C1</smiles>

Following GP4, 1-Boc-3-iodoazetidine $(19 \mu \mathrm{L}, 0.10 \mathrm{mmol})$, boronate $2 \mathbf{n}(0.50 \mathrm{~mL}, 0.50 \mathrm{M}$ in THF, $0.25 \mathrm{mmol}$ ) gave 15 (15 mg, 57\%) as an oil. ${ }^{1} \mathrm{H} \mathrm{NMR}\left(400 \mathrm{MHz}, \mathrm{CDCl}_{3}\right) \delta$ ppm: $7.33-$ $7.14(4 \mathrm{H}, \mathrm{m}), 4.32(2 \mathrm{H}, \mathrm{t}, J=8.7 \mathrm{~Hz}), 3.99-3.89(2 \mathrm{H}, \mathrm{m}), 3.74-3.65(1 \mathrm{H}, \mathrm{m}), 1.47$ (9H, s). Data in accordance with the literature. ${ }^{16}$

tert-Butyl 3-(3-Bromophenyl)azetidine-1-carboxylate (16)

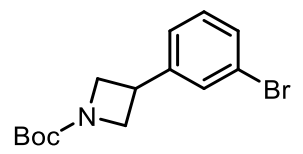

Following GP4, 1-Boc-3-iodoazetidine $(19 \mu \mathrm{L}, 0.10 \mathrm{mmol})$, boronate $20(0.50 \mathrm{~mL}, 0.50 \mathrm{M}$ in THF, $0.25 \mathrm{mmol})$ gave $16(17 \mathrm{mg}, 56 \%)$ as an oil. ${ }^{1} \mathrm{H} \mathrm{NMR}\left(500 \mathrm{MHz}, \mathrm{CDCl}_{3}\right) \delta 7.46(1 \mathrm{H}, \mathrm{t}, J$ $=1.7 \mathrm{~Hz}), 7.39(1 \mathrm{H}, \mathrm{dt}, J=7.1,1.9 \mathrm{~Hz}), 7.26-7.20(2 \mathrm{H}, \mathrm{m}), 4.32(2 \mathrm{H}, \mathrm{t}, J=8.7 \mathrm{~Hz}), 3.94(2 \mathrm{H}$, $\mathrm{dd}, J=8.6,5.9 \mathrm{~Hz}), 3.74-3.62(1 \mathrm{H}, \mathrm{m}), 1.47(9 \mathrm{H}, \mathrm{s}) .{ }^{13} \mathrm{C} \mathrm{NMR}\left(126 \mathrm{MHz}, \mathrm{CDCl}_{3}\right) \delta 156.5$, 144.8, 130.4, 130.3, 130.1, 125.6, 123.0, 79.9, 56.5, 33.3, 28.6; HRMS (ESI) found: $\mathrm{MNa}^{+}$ 334.0405, $\mathrm{C}_{14} \mathrm{H}_{18} \mathrm{O}_{2} \mathrm{NBrNa}$ requires 334.0400.

tert-Butyl 3-(3-(Trifluoromethyl)phenyl)azetidine-1-carboxylate (17)

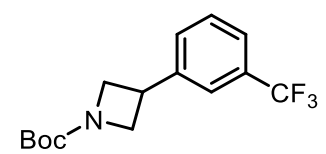

Following GP4, 1-Boc-3-iodoazetidine $(19 \mu \mathrm{L}, 0.10 \mathrm{mmol})$, boronate $2 \mathbf{p}(0.50 \mathrm{~mL}, 0.50 \mathrm{M}$ in THF, $0.25 \mathrm{mmol}$ ) gave 17 (16 mg, 54\%) as an oil. ${ }^{1} \mathrm{H}$ NMR (400 MHz, $\left.\mathrm{CDCl}_{3}\right) \delta$ ppm: $7.55-$ $7.45(4 \mathrm{H}, \mathrm{m}), 4.36(2 \mathrm{H}, \mathrm{t}, J=8.8 \mathrm{~Hz}), 3.99-3.93(2 \mathrm{H}, \mathrm{m}), 3.81-3.74(1 \mathrm{H}, \mathrm{m}), 1.47$ (9H, s). Data in accordance with the literature. ${ }^{18}$ 
tert-Butyl 3-(2-Methoxyphenyl)azetidine-1-carboxylate (18)<smiles>COc1ccccc1C1CN(C(=O)OC(C)(C)C)C1</smiles>

Following GP4, 1-Boc-3-iodoazetidine (19 $\mu \mathrm{L}, 0.10 \mathrm{mmol})$, boronate $2 \mathbf{q}(0.50 \mathrm{~mL}, 0.50 \mathrm{M}$ in THF, $0.25 \mathrm{mmol})$ gave 18 (18 mg, 70\%) as an oil. ${ }^{1} \mathrm{H} \mathrm{NMR}\left(400 \mathrm{MHz}, \mathrm{CDCl}_{3}\right) \delta 7.26-7.20$ $(2 \mathrm{H}, \mathrm{m}), 7.00-6.90(1 \mathrm{H}, \mathrm{m}), 6.88-6.83(1 \mathrm{H}, \mathrm{m}), 4.26(2 \mathrm{H}, \mathrm{t}, J=8.3 \mathrm{~Hz}), 4.12-3.90(3 \mathrm{H}, \mathrm{m})$, $3.81(3 \mathrm{H}, \mathrm{s}), 1.46(9 \mathrm{H}, \mathrm{s})$. Data in accordance with the literature. ${ }^{19}$

tert-Butyl 3-(4-Fluoro-2-methylphenyl)azetidine-1-carboxylate (19)<smiles>Cc1cc(F)ccc1C1CN(C(=O)OC(C)(C)C)C1</smiles>

Following GP4, 1-Boc-3-iodoazetidine $(19 \mu \mathrm{L}, 0.10 \mathrm{mmol})$, boronate $2 \mathbf{r}(0.50 \mathrm{~mL}, 0.50 \mathrm{M}$ in THF, $0.25 \mathrm{mmol})$ gave $19(18 \mathrm{mg}, 68 \%)$ as an oil. ${ }^{1} \mathrm{H} \mathrm{NMR}\left(500 \mathrm{MHz}, \mathrm{CDCl}_{3}\right) \delta 7.29(1 \mathrm{H}$, dd, $J=8.6,5.7 \mathrm{~Hz}), 6.92(1 \mathrm{H}, \mathrm{td}, J=8.4,2.8 \mathrm{~Hz}), 6.87(1 \mathrm{H}, \mathrm{dd}, J=9.6,2.8 \mathrm{~Hz}), 4.30(2 \mathrm{H}, \mathrm{t}, J=$ $8.4 \mathrm{~Hz}), 3.98(2 \mathrm{H}, \mathrm{t}, J=7.5 \mathrm{~Hz}), 3.93-3.83(1 \mathrm{H}, \mathrm{m}), 2.20(3 \mathrm{H}, \mathrm{s}), 1.46(9 \mathrm{H}, \mathrm{s}) ;{ }^{13} \mathrm{C}$ NMR $(126$ $\left.\mathrm{MHz}, \mathrm{CDCl}_{3}\right) \delta 161.6(\mathrm{~d}, J=244.8 \mathrm{~Hz}), 156.6,138.3(\mathrm{~d}, J=7.7 \mathrm{~Hz}), 135.3(\mathrm{~d}, J=3.0 \mathrm{~Hz})$, $127.0(\mathrm{~d}, J=8.3 \mathrm{~Hz}), 117.2(\mathrm{~d}, J=21.0 \mathrm{~Hz}), 112.95(\mathrm{~d}, J=21.0 \mathrm{~Hz}), 79.7,55.1,30.3,28.5$, $19.7(\mathrm{~d}, J=1.7 \mathrm{~Hz}) ;{ }^{19} \mathrm{~F}$ NMR $\left(376 \mathrm{MHz}, \mathrm{CDCl}_{3}\right) \delta-116.8$; HRMS (ESI) found: $\mathrm{MNa}^{+}$ 288.1362, $\mathrm{C}_{15} \mathrm{H}_{20} \mathrm{O}_{2} \mathrm{NFNa}$ requires 288.1370.

tert-Butyl 3-(Benzo[d][1,3]dioxol-5-yl)azetidine-1-carboxylate (20)

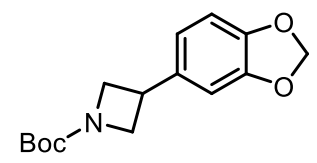

Following GP4, 1-Boc-3-iodoazetidine $(19 \mu \mathrm{L}, 0.10 \mathrm{mmol})$, boronate $2 \mathrm{~s}(0.50 \mathrm{~mL}, 0.50 \mathrm{M}$ in THF, $0.25 \mathrm{mmol})$ gave $20(19 \mathrm{mg}, 68 \%)$ as an oil. ${ }^{1} \mathrm{H} \mathrm{NMR}\left(500 \mathrm{MHz}, \mathrm{CDCl}_{3}\right) \delta 6.84(1 \mathrm{H}, \mathrm{d}$, $J=1.5 \mathrm{~Hz}), 6.79-6.70(2 \mathrm{H}, \mathrm{m}), 5.95(2 \mathrm{H}, \mathrm{s}), 4.29(2 \mathrm{H}, \mathrm{t}, J=8.7 \mathrm{~Hz}), 3.92-3.88(2 \mathrm{H}, \mathrm{m}), 3.68-$ $3.59(1 \mathrm{H}, \mathrm{m}), 1.46(9 \mathrm{H}, \mathrm{s})$. Data in accordance with the literature. ${ }^{20}$ 
tert-Butyl 3-(Benzofuran-5-yl)azetidine-1-carboxylate (21)

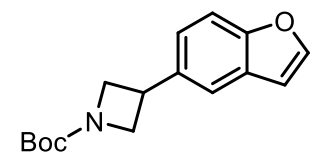

Following GP4, 1-Boc-3-iodoazetidine $(19 \mu \mathrm{L}, 0.10 \mathrm{mmol})$, boronate $2 \mathrm{t}(0.50 \mathrm{~mL}, 0.50 \mathrm{M}$ in THF, $0.25 \mathrm{mmol})$ gave 21 (20 mg, 75\%) as an oil. ${ }^{1} \mathrm{H}$ NMR $\left(400 \mathrm{MHz}, \mathrm{CDCl}_{3}\right) \delta 7.63(1 \mathrm{H}, \mathrm{d}$, $J=2.1 \mathrm{~Hz}), 7.56-7.52(1 \mathrm{H}, \mathrm{m}), 7.48(1 \mathrm{H}, \mathrm{d}, J=8.5 \mathrm{~Hz}), 7.25(1 \mathrm{H}, \mathrm{d}, J=7.6 \mathrm{~Hz}), 6.77-6.72$ $(1 \mathrm{H}, \mathrm{m}), 4.37(2 \mathrm{H}, \mathrm{t}, J=8.6 \mathrm{~Hz}), 4.01(2 \mathrm{H}, \mathrm{dd}, J=8.5,6.1 \mathrm{~Hz}), 3.82(1 \mathrm{H}, \mathrm{tt}, J=8.7,6.1 \mathrm{~Hz})$, $1.48(9 \mathrm{H}, \mathrm{s}) ;{ }^{13} \mathrm{C} \mathrm{NMR}\left(101 \mathrm{MHz}, \mathrm{CDCl}_{3}\right) \delta$ 156.6, 154.2, 145.7, 137.0, 127.9, 123.3, 119.3, 111.7, 106.6, 79.7, 57.3, 33.7, 28.6; HRMS (ESI) found: $\mathrm{MNa}^{+} 296.1243, \mathrm{C}_{16} \mathrm{H}_{19} \mathrm{NO}_{3} \mathrm{Na}$ requires 296.1263 .

tert-Butyl 3-(Benzo[b]thiophen-5-yl)azetidine-1-carboxylat (22)

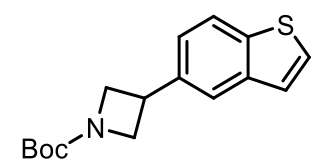

Following GP4, 1-Boc-3-iodoazetidine $(19 \mu \mathrm{L}, 0.10 \mathrm{mmol})$, boronate $2 \mathbf{u}(0.50 \mathrm{~mL}, 0.50 \mathrm{M}$ in THF, $0.25 \mathrm{mmol})$ gave $22(17 \mathrm{mg}, 60 \%)$ as an oil. ${ }^{1} \mathrm{H} \mathrm{NMR}\left(400 \mathrm{MHz}, \mathrm{CDCl}_{3}\right) \delta 7.86(1 \mathrm{H}, \mathrm{d}$, $J=8.4 \mathrm{~Hz}), 7.76(1 \mathrm{H}, \mathrm{d}, J=1.8 \mathrm{~Hz}), 7.46(1 \mathrm{H}, \mathrm{d}, J=5.4 \mathrm{~Hz}), 7.35-7.29(2 \mathrm{H}, \mathrm{m}), 4.38(2 \mathrm{H}, \mathrm{t}$, $J=8.7 \mathrm{~Hz}), 4.03(2 \mathrm{H}, \mathrm{dd}, J=8.6,6.0 \mathrm{~Hz}), 3.85(1 \mathrm{H}$, br. s) $1.48(9 \mathrm{H}, \mathrm{s})$. Data in accordance with the literature. ${ }^{18}$

tert-Butyl 3-(1-(tert-Butoxycarbonyl)azetidin-3-yl)-1H-indole-1-carboxylate (23)

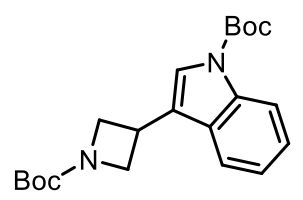

Following GP4, 1-Boc-3-iodoazetidine $(19 \mu \mathrm{L}, 0.10 \mathrm{mmol})$, boronate $2 \mathbf{v}(0.50 \mathrm{~mL}, 0.50 \mathrm{M}$ in THF, $0.25 \mathrm{mmol})$ gave $23(21 \mathrm{mg}, 56 \%)$ as an oil. ${ }^{1} \mathrm{H} \mathrm{NMR}\left(500 \mathrm{MHz}, \mathrm{CDCl}_{3}\right) \delta 8.15(1 \mathrm{H}, \mathrm{d}$, $J=8.2 \mathrm{~Hz}), 7.53-7.45(2 \mathrm{H}, \mathrm{m}), 7.34(1 \mathrm{H}, \mathrm{ddd}, J=8.4,7.2,1.2 \mathrm{~Hz}), 7.26-7.22(1 \mathrm{H}, \mathrm{m}), 4.37$ $(2 \mathrm{H}, \mathrm{t}, J=8.6 \mathrm{~Hz}), 4.11(2 \mathrm{H}, \mathrm{dd}, J=8.5,6.1 \mathrm{~Hz}), 3.95-3.87(1 \mathrm{H}, \mathrm{m}), 1.67(9 \mathrm{H}, \mathrm{s}), 1.47(9 \mathrm{H}$, s); ${ }^{13} \mathrm{C} \mathrm{NMR}\left(126 \mathrm{MHz}, \mathrm{CDCl}_{3}\right) \delta 156.6,149.8,136.0,129.3,124.9,122.8,122.4,121.4,119.1$, 115.7, 83.9, 79.8, 55.1, 28.6, 28.4, 25.6; HRMS (ESI) found: $\mathrm{MNa}^{+} 395.1931, \mathrm{C}_{21} \mathrm{H}_{28} \mathrm{O}_{4} \mathrm{~N}_{2} \mathrm{Na}$ requires 395.1941 . 
tert-Butyl 3-(1-Methyl-1H-indazol-4-yl)azetidine-1-carboxylate (24)

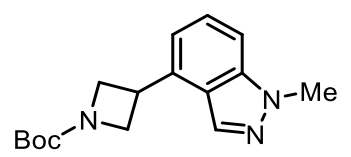

Following GP4, 1-Boc-3-iodoazetidine $(19 \mu \mathrm{L}, 0.10 \mathrm{mmol})$, boronate $2 \mathbf{w}(0.50 \mathrm{~mL}, 0.50 \mathrm{M}$ in THF, $0.25 \mathrm{mmol})$ gave $24(16 \mathrm{mg}, 56 \%)$ as an oil. ${ }^{1} \mathrm{H} \mathrm{NMR}\left(500 \mathrm{MHz}, \mathrm{CDCl}_{3}\right) \delta 8.02(1 \mathrm{H}, \mathrm{s})$, $7.36(1 \mathrm{H}, \mathrm{dd}, J=8.5,6.8 \mathrm{~Hz}), 7.31(1 \mathrm{H}, \mathrm{d}, J=8.4 \mathrm{~Hz}), 7.05(1 \mathrm{H}, \mathrm{dt}, J=6.9,0.9 \mathrm{~Hz}), 4.43(2 \mathrm{H}$, t, $J=8.7 \mathrm{~Hz}), 4.21(2 \mathrm{H}, \mathrm{dd}, J=8.4,6.0 \mathrm{~Hz}), 4.11-4.04(1 \mathrm{H}, \mathrm{m}), 4.09(3 \mathrm{H}, \mathrm{s}), 1.48(9 \mathrm{H}, \mathrm{s}) ;{ }^{13} \mathrm{C}$ NMR (126 MHz, $\left.\mathrm{CDCl}_{3}\right) \delta 156.6,140.4,135.5,131.0,126.5,122.5,118.3,108.1,79.8,55.6$, 35.9, 32.3, 28.6; HRMS (ESI) found: $\mathrm{MH}^{+} 288.1703, \mathrm{C}_{16} \mathrm{H}_{22} \mathrm{O}_{2} \mathrm{~N}_{3}$ requires 288.1707.

tert-Butyl 3-(Thiophen-2-yl)azetidine-1-carboxylate (25)

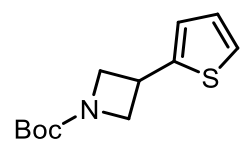

Following GP4, 1-Boc-3-iodoazetidine $(19 \mu \mathrm{L}, 0.10 \mathrm{mmol})$, boronate $2 \mathbf{x}(0.50 \mathrm{~mL}, 0.50 \mathrm{M}$ in THF, $0.25 \mathrm{mmol}$ ) gave 25 (17 mg, 70\%) as an oil. ${ }^{1} \mathrm{H}$ NMR (400 MHz, $\left.\mathrm{CDCl}_{3}\right) \delta$ ppm: 7.22$7.18(1 \mathrm{H}, \mathrm{m}), 6.97-6.92(2 \mathrm{H}, \mathrm{m}), 4.40-4.28(2 \mathrm{H}, \mathrm{m}), 4.03-3.95(3 \mathrm{H}, \mathrm{m}), 1.46(9 \mathrm{H}, \mathrm{s})$. Data in accordance with the literature. ${ }^{21}$

tert-Butyl 3-(Furan-3-yl)azetidine-1-carboxylate (26)<smiles>CC(C)(C)OC(=O)N1CC(c2ccoc2)C1</smiles>

Following GP4, 1-Boc-3-iodoazetidine $(19 \mu \mathrm{L}, 0.10 \mathrm{mmol})$, boronate $2 \mathbf{y}(0.50 \mathrm{~mL}, 0.50 \mathrm{M}$ in THF, $0.25 \mathrm{mmol})$ gave $26(11 \mathrm{mg}, 50 \%)$ as an oil. ${ }^{1} \mathrm{H} \mathrm{NMR}\left(400 \mathrm{MHz}, \mathrm{CDCl}_{3}\right) \delta 7.40(1 \mathrm{H}, \mathrm{m})$, $7.31(1 \mathrm{H}, \mathrm{m}), 6.44-6.39(1 \mathrm{H}, \mathrm{m}), 4.24(2 \mathrm{H}, \mathrm{t}, J=8.5 \mathrm{~Hz}), 3.86(2 \mathrm{H}, \mathrm{dd}, J=8.4,6.0 \mathrm{~Hz}), 3.66-$ $3.62(1 \mathrm{H}, \mathrm{m}), 1.45(9 \mathrm{H}, \mathrm{s})$. Data in accordance with the literature. ${ }^{22}$

tert-Butyl 3-(1-Methyl-1H-pyrazol-4-yl)azetidine-1-carboxylate (27)

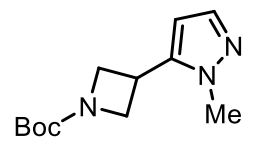

Following GP4, 1-Boc-3-iodoazetidine $(19 \mu \mathrm{L}, 0.10 \mathrm{mmol})$, boronate $\mathbf{2 z}(0.50 \mathrm{~mL}, 0.50 \mathrm{M}$ in THF, $0.25 \mathrm{mmol}$ ) gave 27 (16 mg, 68\%) as an oil. ${ }^{1} \mathrm{H}$ NMR (400 MHz, $\left.\mathrm{CDCl}_{3}\right) \delta 7.41(1 \mathrm{H}, \mathrm{s})$, $7.28(1 \mathrm{H}, \mathrm{s}), 4.26(2 \mathrm{H}, \mathrm{t}, J=8.5 \mathrm{~Hz}), 3.88(3 \mathrm{H}, \mathrm{s}), 3.86-3.80(2 \mathrm{H}, \mathrm{m}), 3.71-3.57(1 \mathrm{H}, \mathrm{m}), 1.45$ 
$(9 \mathrm{H}, \mathrm{s}) ;{ }^{13} \mathrm{C} \mathrm{NMR}\left(101 \mathrm{MHz}, \mathrm{CDCl}_{3}\right) \delta 156.4,137.6,127.7,123.2,79.5,57.0,38.9,28.4,24.3$; HRMS (ESI): Found $\mathrm{MNa}^{+} 260.1362, \mathrm{C}_{12} \mathrm{H}_{19} \mathrm{O}_{2} \mathrm{~N}_{3} \mathrm{Na}$ requires 260.1369.

tert-Butyl 3-(3,5-Dimethylisoxazol-4-yl)azetidine-1-carboxylate (28)

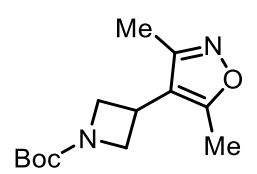

Following GP4, 1-Boc-3-iodoazetidine $(19 \mu \mathrm{L}, 0.10 \mathrm{mmol})$, boronate $2 \mathbf{a a}(0.50 \mathrm{~mL}, 0.50 \mathrm{M}$ in THF, $0.25 \mathrm{mmol})$ gave $28(17 \mathrm{mg}, 66 \%)$ as an oil. ${ }^{1} \mathrm{H} \mathrm{NMR}\left(500 \mathrm{MHz}, \mathrm{CDCl}_{3}\right) \delta 4.27(2 \mathrm{H}, \mathrm{t}, J$ $=8.9 \mathrm{~Hz}), 3.96(2 \mathrm{H}, \mathrm{dd}, J=8.7,6.2 \mathrm{~Hz}), 3.55(1 \mathrm{H}, \mathrm{tt}, J=9.0,6.2 \mathrm{~Hz}), 2.37(3 \mathrm{H}, \mathrm{s}), 2.30(3 \mathrm{H}$, s), $1.46(9 \mathrm{H}, \mathrm{s}) ;{ }^{13} \mathrm{C} \mathrm{NMR}\left(126 \mathrm{MHz}, \mathrm{CDCl}_{3}\right) \delta 165.3,158.9,156.3,113.7,80.0,54.9,28.5$, 23.1, 11.5, 10.8; HRMS (ESI) found: $\mathrm{MNa}^{+} 275.1364, \mathrm{C}_{13} \mathrm{H}_{20} \mathrm{O}_{3} \mathrm{~N}_{2} \mathrm{Na}$ requires 275.1366.

tert-Butyl 3-(6-Chloropyridin-3-yl)azetidine-1-carboxylate (29)<smiles>O=C(c1ccccc1)N1CC(c2ccc(Cl)nc2)C1</smiles>

Following GP4, 1-Boc-3-iodoazetidine $(19 \mu \mathrm{L}, 0.10 \mathrm{mmol})$, boronate $2 \mathbf{a b}(0.50 \mathrm{~mL}, 0.50 \mathrm{M}$ in THF, $0.25 \mathrm{mmol})$ gave $29(12 \mathrm{mg}, 45 \%)$ as an oil. ${ }^{1} \mathrm{H} \mathrm{NMR}\left(400 \mathrm{MHz}, \mathrm{CDCl}_{3}\right) \delta 8.28(1 \mathrm{H}, \mathrm{d}$, $J=2.5 \mathrm{~Hz}), 7.70(1 \mathrm{H}, \mathrm{dd}, J=8.3,2.6 \mathrm{~Hz}), 7.34(1 \mathrm{H}, \mathrm{d}, J=8.3 \mathrm{~Hz}), 4.36(2 \mathrm{H}, \mathrm{t}, J=8.7 \mathrm{~Hz})$, $3.95-3.88(2 \mathrm{H}, \mathrm{m}), 3.72(1 \mathrm{H}, \mathrm{m}), 1.46(9 \mathrm{H}, \mathrm{s})$. Data in accordance with the literature. ${ }^{22}$

tert-Butyl 3-(6-Fluoropyridin-3-yl)azetidine-1-carboxylate (30)

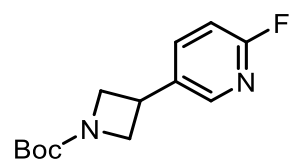

Following GP4, 1-Boc-3-iodoazetidine $(19 \mu \mathrm{L}, 0.10 \mathrm{mmol})$, boronate $2 \mathbf{a c}(0.50 \mathrm{~mL}, 0.50 \mathrm{M}$ in THF, $0.25 \mathrm{mmol})$ gave 30 (13 mg, 50\%) as an oil. ${ }^{1} \mathrm{H}$ NMR (400 MHz, $\left.\mathrm{CDCl}_{3}\right) \delta 8.11(1 \mathrm{H}, \mathrm{br}$ s), 7.84-7.81 (1H, m), 6.99-6.93 (1H, m), 4.37 (2H, t, $J=8.6$ Hz), 3.95-3.88 (2H, m), 3.77$3.73(1 \mathrm{H}, \mathrm{m}), 1.46(9 \mathrm{H}, \mathrm{s})$. Data in accordance with the literature. ${ }^{22}$ 
tert-Butyl 3-(6-Methoxypyridin-3-yl)azetidine-1-carboxylate (31)<smiles>COc1ccc(C2CN(C(=O)OC(C)(C)C)C2)cn1</smiles>

Following GP4, 1-Boc-3-iodoazetidine $(19 \mu \mathrm{L}, 0.10 \mathrm{mmol})$, boronate $2 \mathbf{2 a d}(0.50 \mathrm{~mL}, 0.50 \mathrm{M}$ in THF, $0.25 \mathrm{mmol}$ ) gave 31 (18 mg, 68\%). ${ }^{1} \mathrm{H} \mathrm{NMR}\left(400 \mathrm{MHz}, \mathrm{CD}_{2} \mathrm{Cl}_{2}\right) \delta 8.02(1 \mathrm{H}, \mathrm{d}, J=2.5$ $\mathrm{Hz}), 7.63(1 \mathrm{H}, \mathrm{dd}, J=8.6,2.6 \mathrm{~Hz}), 6.75(1 \mathrm{H}, \mathrm{d}, J=8.6 \mathrm{~Hz}), 4.28(2 \mathrm{H}, \mathrm{t}, J=8.6 \mathrm{~Hz}), 3.94-3.82$ $(5 \mathrm{H}, \mathrm{m}), 3.68(1 \mathrm{H}, \mathrm{tt}, J=8.7,6.0 \mathrm{~Hz}), 1.43(9 \mathrm{H}, \mathrm{s})$. Data in accordance with the literature. ${ }^{22}$

tert-Butyl 3-(2,6-Dimethylpyridin-4-yl)azetidine-1-carboxylate (32)

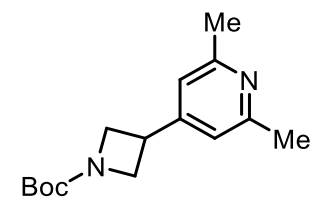

Following GP4, 1-Boc-3-iodoazetidine $(19 \mu \mathrm{L}, 0.10 \mathrm{mmol})$, boronate $2 \mathbf{a e}(0.50 \mathrm{~mL}, 0.50 \mathrm{M}$ in THF, $0.25 \mathrm{mmol}$ ) gave $32(12 \mathrm{mg}, 45 \%)$. ${ }^{1} \mathrm{H} \mathrm{NMR}\left(500 \mathrm{MHz}, \mathrm{CDCl}_{3}\right) \delta 6.94(2 \mathrm{H}, \mathrm{s}), 4.32(2 \mathrm{H}$, $\mathrm{t}, J=8.7 \mathrm{~Hz}), 3.94(2 \mathrm{H}, \mathrm{dd}, J=8.6,5.8 \mathrm{~Hz}), 3.63(1 \mathrm{H}, \mathrm{tt}, J=8.7,5.8 \mathrm{~Hz}), 2.56(6 \mathrm{H}, \mathrm{s}), 1.47$ $(9 \mathrm{H}, \mathrm{s}) ;{ }^{13} \mathrm{C} \mathrm{NMR}\left(126 \mathrm{MHz}, \mathrm{CDCl}_{3}\right){ }^{13} \mathrm{C} \mathrm{NMR}\left(126 \mathrm{MHz}, \mathrm{CDCl}_{3}\right) \delta$ 157.8, 156.3, 118.9, 79.9, 55.3, 32.7, 28.4, 24.0; HRMS (APCI) found: $\mathrm{MH}^{+}$263.1750, $\mathrm{C}_{15} \mathrm{H}_{23} \mathrm{O}_{2} \mathrm{~N}_{2}$ requires 263.1754.

tert-Butyl 3-Vinylazetidine-1-carboxylate (33)

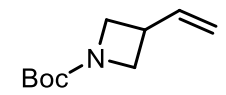

Following GP4, 1-Boc-3-iodoazetidine $(19 \mu \mathrm{L}, 0.10 \mathrm{mmol})$, boronate $2 \mathbf{a f}(0.50 \mathrm{~mL}, 0.50 \mathrm{M}$ in THF, $0.25 \mathrm{mmol})$ gave $33(12 \mathrm{mg}, 65 \%)$ as an oil. ${ }^{1} \mathrm{H}$ NMR $\left(500 \mathrm{MHz}, \mathrm{CDCl}_{3}\right) \delta 5.99(1 \mathrm{H}$, ddd, $J=16.9,10.4,7.8 \mathrm{~Hz}), 5.14-5.02(2 \mathrm{H}, \mathrm{m}), 4.08(2 \mathrm{H}, \mathrm{t}, J=8.5 \mathrm{~Hz}), 3.73(2 \mathrm{H}, \mathrm{dd}, J=8.5$, $5.9 \mathrm{~Hz}), 3.24-3.12(1 \mathrm{H}, \mathrm{m}), 1.43(9 \mathrm{H}, \mathrm{s}) ;{ }^{13} \mathrm{C} \mathrm{NMR}\left(126 \mathrm{MHz}, \mathrm{CDCl}_{3}\right) \delta 156.5,138.9,115.8$, 79.5, 54.5, 32.3, 28.6; HRMS (ESI) found: $\mathrm{MNa}^{+}$206.1149, $\mathrm{C}_{10} \mathrm{H}_{17} \mathrm{O}_{2} \mathrm{NNa}$ requires 206.1152. Data in accordance with the literature. ${ }^{23}$

tert-Butyl (E)-3-Styrylazetidine-1-carboxylate (34)

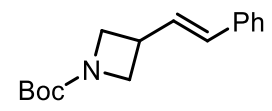

Following GP4, 1-Boc-3-iodoazetidine $(19 \mu \mathrm{L}, 0.10 \mathrm{mmol})$, boronate $2 \mathrm{ag}(0.50 \mathrm{~mL}, 0.50 \mathrm{M}$ in THF, $0.25 \mathrm{mmol}$ ) gave $34(23 \mathrm{mg}, 90 \%)$ as an oil. ${ }^{1} \mathrm{H}$ NMR (400 $\left.\mathrm{MHz}, \mathrm{CDCl}_{3}\right) \delta: 7.38-7.20$ 
$(5 \mathrm{H}, \mathrm{m}), 6.49-6.31(2 \mathrm{H}, \mathrm{m}), 4.16(2 \mathrm{H}, \mathrm{t}, J=8.5 \mathrm{~Hz}), 3.83(2 \mathrm{H}, \mathrm{dd}, J=8.6,5.9 \mathrm{~Hz}), 3.41-3.29$ $(1 \mathrm{H}, \mathrm{m}), 1.46(9 \mathrm{H}, \mathrm{s})$. Data in accordance with the literature. ${ }^{24}$

tert-Butyl (E)-3-(Hex-1-en-1-yl)azetidine-1-carboxylate (35)

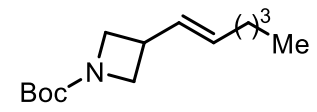

Following GP4, 1-Boc-3-iodoazetidine $(19 \mu \mathrm{L}, 0.10 \mathrm{mmol})$, boronate 2ah $(0.50 \mathrm{~mL}, 0.50 \mathrm{M}$ in THF, $0.25 \mathrm{mmol}$ ) gave 35 (23 mg, 98\%) as an oil. ${ }^{1} \mathrm{H} \mathrm{NMR}\left(400 \mathrm{MHz}, \mathrm{CDCl}_{3}\right) \delta 5.61-5.40$ $(2 \mathrm{H}, \mathrm{m}), 4.04(2 \mathrm{H}, \mathrm{t}, J=8.4 \mathrm{~Hz}), 3.67(2 \mathrm{H}, \mathrm{dd}, J=8.5,6.0 \mathrm{~Hz}), 3.16-3.07(1 \mathrm{H}, \mathrm{m}), 2.05-1.95$ $(2 \mathrm{H}, \mathrm{m}), 1.41(9 \mathrm{H}, \mathrm{s}), 1.37-1.10(4 \mathrm{H}, \mathrm{m}), 0.88(3 \mathrm{H}, \mathrm{t}, J=6.3 \mathrm{~Hz})$. Data in accordance with the literature. $^{25}$

tert-Butyl (E)-3-(3-Methoxyprop-1-en-1-yl)azetidine-1-carboxylate (36)<smiles>COC/C=C/C1CN(C(=O)c2ccccc2)C1</smiles>

Following GP4, 1-Boc-3-iodoazetidine $(19 \mu \mathrm{L}, 0.10 \mathrm{mmol})$, boronate 2ai $(0.50 \mathrm{~mL}, 0.50 \mathrm{M}$ in THF, $0.25 \mathrm{mmol})$ gave $36(22 \mathrm{mg}, 99 \%)$ as an oil. ${ }^{1} \mathrm{H} \mathrm{NMR}\left(400 \mathrm{MHz}, \mathrm{CDCl}_{3}\right) \delta 5.88(1 \mathrm{H}$, ddt, $J=15.5,8.0,1.4 \mathrm{~Hz}), 5.63(1 \mathrm{H}, \mathrm{dt}, J=15.5,5.9 \mathrm{~Hz}), 4.07(2 \mathrm{H}, \mathrm{t}, J=8.5 \mathrm{~Hz}), 3.89(2 \mathrm{H}, \mathrm{d}, J=$ $\mathrm{Hz}), 3.73(2 \mathrm{H}, \mathrm{dd}, J=8.5,6.0 \mathrm{~Hz}), 3.33(3 \mathrm{H}, \mathrm{s}), 3.26-3.10(1 \mathrm{H}, \mathrm{m}), 1.43(9 \mathrm{H}, \mathrm{s}) ;{ }^{13} \mathrm{C} \mathrm{NMR}$ $\left(101 \mathrm{MHz}, \mathrm{CDCl}_{3}\right) \delta 156.5,134.0,127.9,79.5,72.7,58.2,54.7,31.2,28.5$; HRMS (ESI) found: $\mathrm{MNa}^{+} 250.1407, \mathrm{C}_{12} \mathrm{H}_{21} \mathrm{O}_{3} \mathrm{NNa}$ requires 250.1414.

tert-Butyl (E)-3-(3-((tert-Butyldimethylsilyl)oxy)prop-1-en-1-yl)azetidine-1-carboxylate (37)

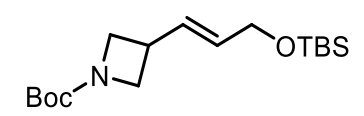

Following GP4, 1-Boc-3-iodoazetidine ( $19 \mu \mathrm{L}, 0.10 \mathrm{mmol})$, boronate 2aj (0.50 mL, 0.50 M in THF, $0.25 \mathrm{mmol})$ gave $37(32 \mathrm{mg}, 99 \%)$ as an oil. ${ }^{1} \mathrm{H} \mathrm{NMR}\left(500 \mathrm{MHz}, \mathrm{CDCl}_{3}\right) \delta 5.83(1 \mathrm{H}$, dd, $J=15.3,8.1 \mathrm{~Hz}), 5.61(1 \mathrm{H}, \mathrm{dtd}, J=15.2,4.9,1.1 \mathrm{~Hz}), 4.15(2 \mathrm{H}, \mathrm{ddd}, J=5.0,1.7,0.8 \mathrm{~Hz}), 4.07$ $(2 \mathrm{H}, \mathrm{t}, J=8.5 \mathrm{~Hz}), 3.72(2 \mathrm{H}, \mathrm{dd}, J=8.5,6.0 \mathrm{~Hz}), 3.25-3.14(1 \mathrm{H}, \mathrm{m}), 1.44(9 \mathrm{H}, \mathrm{s}), 0.91(9 \mathrm{H}$, s), $0.07(6 \mathrm{H}, \mathrm{s}) ;{ }^{13} \mathrm{C} \mathrm{NMR}\left(126 \mathrm{MHz}, \mathrm{CDCl}_{3}\right) \delta 156.5,131.0,130.8,79.5,63.5,54.8,31.1,28.6$, 26.1, 18.6, -5.0; HRMS (ESI) found: $2 \mathrm{MH}^{+} 655.4527, \mathrm{C}_{34} \mathrm{H}_{66} \mathrm{O}_{6} \mathrm{~N}_{2} \mathrm{Si}_{2} \mathrm{H}$ requires 655.4532. 
tert-Butyl 3-(3,6-Dihydro-2H-pyran-4-yl)azetidine-1-carboxylate (38)

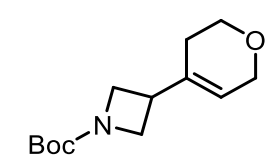

Following GP4, 1-Boc-3-iodoazetidine $(19 \mu \mathrm{L}, 0.10 \mathrm{mmol})$, boronate 2ak $(0.50 \mathrm{~mL}, 0.50 \mathrm{M}$ in THF, $0.25 \mathrm{mmol})$ gave 38 (13 mg, 99\%) as an oil. ${ }^{1} \mathrm{H} \mathrm{NMR}\left(500 \mathrm{MHz}, \mathrm{CDCl}_{3}\right) \delta 5.56-5.53$ $(1 \mathrm{H}, \mathrm{m}), 4.17-4.14(2 \mathrm{H}, \mathrm{m}), 4.02(2 \mathrm{H}, \mathrm{t}, J=8.6 \mathrm{~Hz}), 3.86-3.78(4 \mathrm{H}, \mathrm{m}), 3.19-3.10(1 \mathrm{H}, \mathrm{m})$, $2.09(2 \mathrm{H}, \mathrm{td}, J=5.3,2.6 \mathrm{~Hz}), 1.44(9 \mathrm{H}, \mathrm{s}) ;{ }^{13} \mathrm{C} \mathrm{NMR}\left(126 \mathrm{MHz}, \mathrm{CDCl}_{3}\right) \delta 156.5,134.6,121.0$, 79.6, 65.5, 64.2, 52.6, 34.3, 28.6, 25.8; HRMS (ESI) found: $\mathrm{MNa}^{+} 262.1409, \mathrm{C}_{13} \mathrm{H}_{21} \mathrm{NO}_{3} \mathrm{Na}$ requires 262.1414. Data in accordance with the literature. ${ }^{26}$

tert-Butyl 3-(2-Methylprop-1-en-1-yl)azetidine-1-carboxylate (39)<smiles>CC(C)=CC1CN(C(=O)OC(C)(C)C)C1</smiles>

Following GP4, 1-Boc-3-iodoazetidine $(19 \mu \mathrm{L}, 0.10 \mathrm{mmol})$, boronate $2 \mathrm{al}(0.50 \mathrm{~mL}, 0.50 \mathrm{M}$ in THF, $0.25 \mathrm{mmol})$ gave $39(15 \mathrm{mg}, 72 \%)$ as an oil. ${ }^{1} \mathrm{H} \mathrm{NMR}\left(400 \mathrm{MHz}, \mathrm{CDCl}_{3}\right) \delta 5.35(1 \mathrm{H}, \mathrm{dq}$, $J=9.0,1.4 \mathrm{~Hz}), 4.09(2 \mathrm{H}, \mathrm{t}, J=8.4 \mathrm{~Hz}), 3.63(2 \mathrm{H}, \mathrm{dd}, J=8.3,6.1 \mathrm{~Hz}), 3.44-3.27(1 \mathrm{H}, \mathrm{m})$, $1.70(3 \mathrm{H}, \mathrm{s}), 1.57(3 \mathrm{H}, \mathrm{s}), 1.43(9 \mathrm{H}, \mathrm{s}) ;{ }^{13} \mathrm{C} \mathrm{NMR}\left(101 \mathrm{MHz}, \mathrm{CDCl}_{3}\right) \delta 156.5,134.1,126.3$, 79.3, 55.9, 28.6, 27.5, 25.7, 18.2; HRMS (ESI) found: $\mathrm{MNa}^{+} 234.1454, \mathrm{C}_{12} \mathrm{H}_{21} \mathrm{O}_{2} \mathrm{NNa}$ requires 234.1465 .

tert-Butyl 3-(Phenylethynyl)azetidine-1-carboxylate (40)

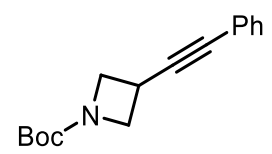

Following GP4, 1-Boc-3-iodoazetidine $(19 \mu \mathrm{L}, 0.10 \mathrm{mmol})$, boronate $2 \mathrm{am}(0.50 \mathrm{~mL}, 0.50 \mathrm{M}$ in THF, $0.25 \mathrm{mmol})$ gave 40 (9 mg, 34\%) as an oil. ${ }^{1} \mathrm{H}$ NMR (400 MHz, $\left.\mathrm{CDCl}_{3}\right) \delta 7.43-7.38$ $(2 \mathrm{H}, \mathrm{m}), 7.33-7.28(3 \mathrm{H}, \mathrm{m}), 4.21(2 \mathrm{H}, \mathrm{t}, J=8.4 \mathrm{~Hz}), 4.02(2 \mathrm{H}, \mathrm{dd}, J=8.1,6.4 \mathrm{~Hz}), 3.54(1 \mathrm{H}$, $\mathrm{tt}, J=8.6,6.4 \mathrm{~Hz}), 1.45(9 \mathrm{H}, \mathrm{s})$. Data in accordance with the literature. ${ }^{24}$

tert-Butyl 3-Benzylazetidine-1-carboxylate (41)

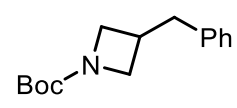

Following GP4 but using cumOOSit-BuPh $2(117 \mathrm{mg}, 0.300 \mathrm{mmol})$ as oxidant, 1-Boc-3iodoazetidine $(19 \mu \mathrm{L}, 0.10 \mathrm{mmol})$, boronate $2 \mathrm{an}(0.50 \mathrm{~mL}, 0.50 \mathrm{M}$ in THF, $0.25 \mathrm{mmol})$ gave 
$41(14 \mathrm{mg}, 57 \%)$ as an oil. ${ }^{1} \mathrm{H}$ NMR $\left(500 \mathrm{MHz}, \mathrm{CDCl}_{3}\right) \delta 7.33-7.27(2 \mathrm{H}, \mathrm{m}), 7.23-7.19(1 \mathrm{H}$, m) 7.18-7.11 (2H, m), $3.99(2 \mathrm{H}, \mathrm{t}, J=8.3 \mathrm{~Hz}), 3.65(2 \mathrm{H}, \mathrm{dd}, J=8.7,5.4 \mathrm{~Hz}), 2.90(2 \mathrm{H}, \mathrm{d}, J=$ $8.0 \mathrm{~Hz},), 2.81(1 \mathrm{H}, \mathrm{pt}, J=8.1,5.4 \mathrm{~Hz}), 1.44(9 \mathrm{H}, \mathrm{s},) .{ }^{13} \mathrm{C} \mathrm{NMR}\left(126 \mathrm{MHz}, \mathrm{CDCl}_{3}\right) \delta 156.6$, $139.5,128.7,128.5,126.5,79.4,54.4,40.4,30.0,28.6$. Data in accordance with the literature. ${ }^{27}$

tert-Butyl 3-(3-Chlorobenzyl)azetidine-1-carboxylate (42)

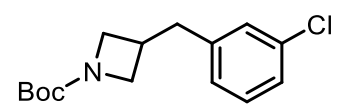

Following GP4 but using cumOOSit-BuPh $2 ~(117 \mathrm{mg}, 0.300 \mathrm{mmol})$ as oxidant, 1-Boc-3iodoazetidine $(19 \mu \mathrm{L}, 0.10 \mathrm{mmol})$, boronate $2 \mathbf{a o}(0.50 \mathrm{~mL}, 0.50 \mathrm{M}$ in THF, $0.25 \mathrm{mmol})$ gave $42(17 \mathrm{mg}, 62 \%)$ as a solid. ${ }^{1} \mathrm{H}$ NMR $\left(500 \mathrm{MHz}, \mathrm{CDCl}_{3}\right) \delta 7.24-7.17(2 \mathrm{H}, \mathrm{m}), 7.14-7.12(1 \mathrm{H}$, m), $7.02(1 \mathrm{H}, \mathrm{dt}, J=7.1,1.7 \mathrm{~Hz}), 3.99(2 \mathrm{H}, \mathrm{t}, J=8.3 \mathrm{~Hz}), 3.63(2 \mathrm{H}, \mathrm{dd}, J=8.6,5.3 \mathrm{~Hz}), 2.88$ $(2 \mathrm{H}, \mathrm{d}, J=8.0 \mathrm{~Hz}), 2.84-2.73(1 \mathrm{H}, \mathrm{m}), 1.44(9 \mathrm{H}, \mathrm{s}) .{ }^{13} \mathrm{C} \mathrm{NMR}\left(126 \mathrm{MHz}, \mathrm{CDCl}_{3}\right) \delta 156.6$, 141.5, 134.5, 130.0, 128.7, 126.8, 126.7, 79.5, 54.2, 40.0, 29.8, 28.6. HRMS (ESI) found: $\mathrm{MNa}^{+}$ 304.1067, $\mathrm{C}_{15} \mathrm{H}_{20} \mathrm{O}_{2} \mathrm{NClNa}$ requires 304.1075.

tert-Butyl 3-(3-(Trifluoromethyl)benzyl)azetidine-1-carboxylate (43)

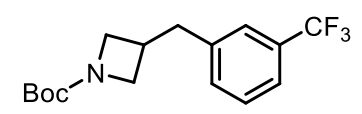

Following GP4 but using cumOOSit-BuPh $2(117 \mathrm{mg}, 0.300 \mathrm{mmol})$ as oxidant, 1-Boc-3iodoazetidine $(19 \mu \mathrm{L}, 0.10 \mathrm{mmol})$, boronate $2 \mathbf{a p}(0.50 \mathrm{~mL}, 0.50 \mathrm{M}$ in THF, $0.25 \mathrm{mmol})$ gave $43(20 \mathrm{mg}, 64 \%)$ as a solid. ${ }^{1} \mathrm{H}$ NMR $\left(500 \mathrm{MHz}, \mathrm{CDCl}_{3}\right) \delta 7.48(1 \mathrm{H}, \mathrm{d}, J=7.8 \mathrm{~Hz}), 7.44-7.38$ $(2 \mathrm{H}, \mathrm{m}), 7.33(1 \mathrm{H}, \mathrm{d}, J=7.6 \mathrm{~Hz}), 4.00(2 \mathrm{H}, \mathrm{t}, J=8.3 \mathrm{~Hz}), 3.64(2 \mathrm{H}, \mathrm{dd}, J=8.6,5.3 \mathrm{~Hz}), 2.97$ $(2 \mathrm{H}, \mathrm{d}, J=8.0 \mathrm{~Hz}), 2.88-2.77(1 \mathrm{H}, \mathrm{m}), 1.44(9 \mathrm{H}, \mathrm{s}) ;{ }^{13} \mathrm{C} \mathrm{NMR}\left(126 \mathrm{MHz}, \mathrm{CDCl}_{3}\right) \delta 156.6$, $140.4,131.9,131.1$ (q, $J=31.9 \mathrm{~Hz}), 129.2,125.2(\mathrm{q}, J=3.8 \mathrm{~Hz}), 124.2(\mathrm{q}, J=271.9 \mathrm{~Hz}), 123.5$ $(\mathrm{q}, J=3.7 \mathrm{~Hz}), 79.6,53.9,40.1,29.8,28.6 ;{ }^{19} \mathrm{~F} \mathrm{NMR}\left(376 \mathrm{MHz}, \mathrm{CDCl}_{3}\right) \delta-62.6 ; \mathrm{HRMS}(\mathrm{ESI})$ found: $\mathrm{MNa}^{+} 338.1328, \mathrm{C}_{16} \mathrm{H}_{20} \mathrm{O}_{2} \mathrm{NF}_{3} \mathrm{Na}$ requires 338.1338.

tert-Butyl 3-Allylazetidine-1-carboxylate (44)

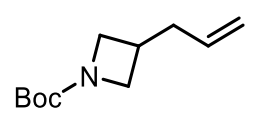

Following GP4, 1-Boc-3-iodoazetidine $(19 \mu \mathrm{L}, 0.10 \mathrm{mmol})$, boronate $2 \mathrm{aq}(0.50 \mathrm{~mL}, 0.50 \mathrm{M}$ in THF, $0.25 \mathrm{mmol})$ gave $44(13 \mathrm{mg}, 65 \%)$ as an oil. ${ }^{1} \mathrm{H} \mathrm{NMR}\left(400 \mathrm{MHz}, \mathrm{CDCl}_{3}\right) \delta 5.73(1 \mathrm{H}$, ddt, $J=17.6,9.8,6.5 \mathrm{~Hz}), 5.08-4.97(2 \mathrm{H}, \mathrm{m}), 3.99(2 \mathrm{H}, \mathrm{dd}, J=8.7,8.0 \mathrm{~Hz}), 3.56(2 \mathrm{H}, \mathrm{dd}, J=8.5$, 
$5.4 \mathrm{~Hz}), 2.58(1 \mathrm{H}, \mathrm{tt}, J=7.9,5.4 \mathrm{~Hz}), 2.32(2 \mathrm{H}, \mathrm{ddt}, J=7.8,6.5,1.4 \mathrm{~Hz}), 1.43(9 \mathrm{H}, \mathrm{s}) ;{ }^{13} \mathrm{C}$ NMR $\left(126 \mathrm{MHz}, \mathrm{CDCl}_{3}\right) \delta 156.6,135.2,116.5,79.3,54.1,38.3,28.6,28.0$; HRMS (ESI) found $\mathrm{MNa}^{+} 220.1303, \mathrm{C}_{11} \mathrm{H}_{19} \mathrm{O}_{2} \mathrm{NNa}$ requires 220.1308.

\section{tert-Butyl 4-Benzylpiperidine-1-carboxylate (47)}

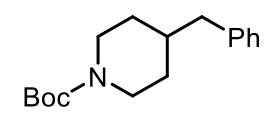

Following GP4 but using $\left[\mathrm{Cu}(\mathrm{MeCN})_{4}\right] \mathrm{PF}_{6}(4 \mathrm{mg}, 10 \% \mathrm{~mol})$, cumOOTES (80 mg, $85 \mu \mathrm{L}, 0.30$ mmol, 3.0 equiv.) and no TMHD, tert-butyl 4-(iodomethyl)piperidine-1-carboxylate (33 mg, $0.10 \mathrm{mmol})$ and boronate $2 \mathrm{a}(0.50 \mathrm{~mL}, 0.50 \mathrm{M}$ in THF, $0.25 \mathrm{mmol})$ gave $47(15 \mathrm{mg}, 54 \%)$ as an oil. ${ }^{1} \mathrm{H}$ NMR (400 MHz, $\left.\mathrm{CDCl}_{3}\right) \delta$ 7.31-7.24 (2H, m), 7.23-7.16 (1H, m), 7.16-7.11 (2H, m), 4.06 (2H, br. s), $2.63(2 \mathrm{H}, \mathrm{t}, J=12.4 \mathrm{~Hz}), 2.53(2 \mathrm{H}, \mathrm{d}, J=7.0 \mathrm{~Hz}), 1.69-1.57$ (4H, m), 1.45 $(9 \mathrm{H}, \mathrm{s}), 1.21-1.08(2 \mathrm{H}, \mathrm{m})$. Data in accordance with the literature. ${ }^{28}$

\section{tert-Butyl 4-Phenylpiperidine-1-carboxylate (48)}

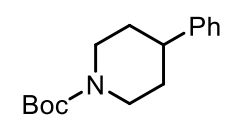

Following GP4 but using cumOOTES ( $80 \mathrm{mg}, 85 \mu \mathrm{L}, 0.30 \mathrm{mmol}, 3.0$ equiv.) and no TMHD, tert-butyl 4-iodopiperidine-1-carboxylate $(31 \mathrm{mg}, 0.10 \mathrm{mmol})$ and boronate $2 \mathbf{a}(0.50 \mathrm{~mL}, 0.50$ $\mathrm{M}$ in THF, $0.25 \mathrm{mmol})$ gave 48 (16 mg, 62\%) as an oil. ${ }^{1} \mathrm{H} \mathrm{NMR}\left(400 \mathrm{MHz}, \mathrm{CDCl}_{3}\right) \delta 7.34$ 7.28(2H, m), 7.24-7.18(3H, m), $4.25(2 \mathrm{H}, \mathrm{d}, \mathrm{J}=13.3 \mathrm{~Hz}), 2.80(2 \mathrm{H}, \mathrm{dt}, \mathrm{J}=13.0,2.6 \mathrm{~Hz}), 2.64$ $(1 \mathrm{H}, \mathrm{tt}, \mathrm{J}=12.2,3.6 \mathrm{~Hz}), 1.86-1.78(2 \mathrm{H}, \mathrm{m}), 1.69-1.56(2 \mathrm{H}, \mathrm{m}), 1.48(9 \mathrm{H}, \mathrm{s})$. Data in accordance with the literature. ${ }^{29}$

\section{2-(4-Phenylpiperidin-1-yl)pyrimidine (49)}<smiles>c1ccc(C2CCN(c3ncccn3)CC2)cc1</smiles>

Following GP4 but using cumOOTES ( $80 \mathrm{mg}, 85 \mu \mathrm{L}, 0.30 \mathrm{mmol}, 3.0$ equiv.) and no TMHD, 2-(4-iodopiperidin-1-yl)pyrimidine $\mathbf{S 1 0}(29 \mathrm{mg}, 0.10 \mathrm{mmol})$ and boronate $\mathbf{2 a}(0.50 \mathrm{~mL}, 0.50 \mathrm{M}$ in THF, $0.25 \mathrm{mmol})$ gave 49 (14 mg, 59\%) as an oil. ${ }^{1} \mathrm{H} \mathrm{NMR}\left(500 \mathrm{MHz}, \mathrm{CDCl}_{3}\right) \delta 8.32(2 \mathrm{H}$, $\mathrm{d}, J=4.7 \mathrm{~Hz}), 7.33-7.29(2 \mathrm{H}, \mathrm{m}), 7.24-7.18(3 \mathrm{H}, \mathrm{m}), 6.47(1 \mathrm{H}, \mathrm{t}, J=4.7 \mathrm{~Hz}), 4.97-4.89$ (2H, m), $2.97(2 \mathrm{H}, \mathrm{td}, J=13.0,2.6 \mathrm{~Hz}), 2.80(1 \mathrm{H}, \mathrm{tt}, J=12.2,3.6 \mathrm{~Hz}), 1.98-1.91(2 \mathrm{H}, \mathrm{m}), 1.71(2 \mathrm{H}$, 
$\mathrm{qd}, J=12.6,4.2 \mathrm{~Hz}) .{ }^{13} \mathrm{C} \mathrm{NMR}\left(126 \mathrm{MHz}, \mathrm{CDCl}_{3}\right) \delta 161.6,157.8,146.0,128.5,126.8,126.3$, 109.4, 44.5, 43.1, 33.1. HRMS (ASAP): Found $\mathrm{MH}^{+} 240.1492, \mathrm{C}_{15} \mathrm{H}_{18} \mathrm{~N}_{3}$ requires 240.1495.

tert-Butyl 3-Phenyl-8-azabicyclo[3.2.1]octane-8-carboxylate (50)

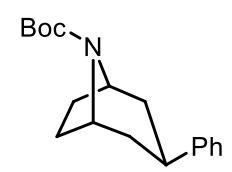

Following GP4 but using cumOOTES ( $80 \mathrm{mg}, 85 \mu \mathrm{L}, 0.30 \mathrm{mmol}, 3.0$ equiv.) and no TMHD, tert-butyl 3-exo-iodo-8-azabicyclo[3.2.1]octane-8-carboxylate $\mathbf{S 7}$ (34 $\mathrm{mg}, 0.10 \mathrm{mmol}$ ) and boronate 2a $(0.50 \mathrm{~mL}, 0.50 \mathrm{M}$ in THF, $0.25 \mathrm{mmol})$ gave $\mathbf{5 0}(16 \mathrm{mg}, 56 \%)$ as an oil. $\mathrm{dr}=1.15: 1$. ${ }^{1} \mathrm{H}$ NMR (400 MHz, $\left.\mathrm{CDCl}_{3}\right) \delta$ 7.33-7.26 (2H, m), 7.25-7.15 (3H, m), 4.39-4.19 (2H, m), 3.08 $(0.5 \mathrm{H}, \mathrm{tt}, J=11.9,5.4 \mathrm{~Hz}), 2.65(0.5 \mathrm{H}, \mathrm{tt}, J=10.4,7.1 \mathrm{~Hz}), 2.56-2.39(1 \mathrm{H}, \mathrm{m}), 2.10-1.90(3 \mathrm{H}$, $\mathrm{m}), 1.87-1.60(4 \mathrm{H}, \mathrm{m}), 1.50(9 \mathrm{H}, \mathrm{s}) .{ }^{13} \mathrm{C} \mathrm{NMR}\left(101 \mathrm{MHz}, \mathrm{CDCl}_{3}\right.$, diastereomers and rotamers) $\delta 154.6,153.6,145.2,144.8,128.5,128.3,127.4,127.1,126.3,126.0,79.14,79.11,54.2,53.4$, 51.6, 51.0, 35.2, 34.3, 31.7, 31.1, 28.5, 28.5, 27.8. HRMS (ESI): Found $\mathrm{MNa}^{+}$310.1768, $\mathrm{C}_{18} \mathrm{H}_{25} \mathrm{O}_{2} \mathrm{NNa}$ requires 310.1768 .

\section{4-Phenyltetrahydro-2H-pyran (51)}

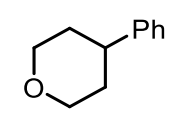

Following GP4 but using cumOOTES ( $80 \mathrm{mg}, 85 \mu \mathrm{L}, 0.30 \mathrm{mmol}, 3.0$ equiv.) and no TMHD, 4-iodotetrahydro-2 $\mathrm{H}$-pyran $(21.2 \mathrm{mg}, 0.10 \mathrm{mmol})$ and boronate $\mathbf{2 a}(0.50 \mathrm{~mL}, 0.50 \mathrm{M}$ in THF, $0.25 \mathrm{mmol})$ gave $51(11 \mathrm{mg}, 66 \%)$ as an oil. ${ }^{1} \mathrm{H} \mathrm{NMR}\left(400 \mathrm{MHz}, \mathrm{CDCl}_{3}\right) \delta 7.33(2 \mathrm{H}, \mathrm{d}, \mathrm{J}=7.4$ $\mathrm{Hz}), 7.26-7.18(3 \mathrm{H}, \mathrm{m}), 4.08(2 \mathrm{H}, \mathrm{dd}, J=11.4,4.3 \mathrm{~Hz}), 3.53(2 \mathrm{H}, \mathrm{td}, J=11.5,2.7 \mathrm{~Hz}), 2.75$ $(1 \mathrm{H}, \mathrm{tt}, J=11.5,4.4 \mathrm{~Hz}), 1.90-1.72(4 \mathrm{H}, \mathrm{m})$. Data in accordance with the literature. ${ }^{30}$

\section{2-(4-Bromophenyl)-4-phenyltetrahydro-2H-pyran (52)}
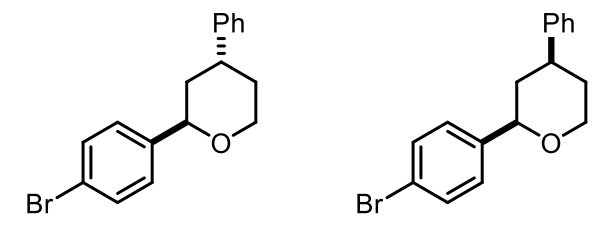

Following GP4 but using cumOOTES ( $80 \mathrm{mg}, 85 \mu \mathrm{L}, 0.30 \mathrm{mmol}, 3.0$ equiv.) and no TMHD, 2-(4-bromophenyl)-4-iodotetrahydro-2H-pyran $\mathbf{S 1 6}$ (37 mg, $0.10 \mathrm{mmol}$ ) and boronate 2a (0.50 $\mathrm{mL}, 0.50 \mathrm{M}$ in THF, $0.25 \mathrm{mmol})$ gave $\mathbf{5 2}(15 \mathrm{mg}, 47 \%)$ as an oil as an unseparable mixture of diastereomers. $\mathrm{dr}=1.8: 1 .{ }^{1} \mathrm{H}$ NMR $\left(500 \mathrm{MHz} \mathrm{CDCl}_{3}\right.$, diastereomers $) \delta 7.51(1.3 \mathrm{H}, \mathrm{d}, J=8.5$ 
Hz), $7.46(0.7 \mathrm{H}, \mathrm{d}, J=8.5 \mathrm{~Hz}), 7.38-7.28(4 \mathrm{H}, \mathrm{m}), 7.28-7.20(3 \mathrm{H}, \mathrm{m}), 4.90(0.65 \mathrm{H}, \mathrm{t}, J=4.9$ Hz), $4.45(0.35 \mathrm{H}, \mathrm{dd}, J=11.1,2.2 \mathrm{~Hz}), 4.29(0.35 \mathrm{H}, \mathrm{ddd}, J=11.6,4.2,2.1 \mathrm{~Hz}), 3.84(0.65 \mathrm{H}$, ddd, $J=11.7,6.0,3.9 \mathrm{~Hz}), 3.81-3.72(1 \mathrm{H}, \mathrm{m}), 3.03(0.65 \mathrm{H}, \mathrm{tt}, J=8.5,4.5 \mathrm{~Hz}), 2.96(0.35 \mathrm{H}, \mathrm{tt}$, $J=11.6,4.1 \mathrm{~Hz}), 2.36-2.25(1.3 \mathrm{H}, \mathrm{m}), 2.10-1.96(1 \mathrm{H}, \mathrm{m}), 1.96-1.81(1.3 \mathrm{H}, \mathrm{m}), 1.75-1.65$ $(0.4 \mathrm{H}, \mathrm{m}) ;{ }^{13} \mathrm{C} \mathrm{NMR}\left(126 \mathrm{MHz}, \mathrm{CDCl}_{3}\right.$, diastereomers) $\delta 145.2,144.6,141.8,140.4,131.6$, 131.4, 128.6, 128.6, 128.2, 127.5, 127.1, 126.7, 126.5, 126.3, 121.1, 120.9, 79.2, 73.4, 68.7, $62.8,42.0,41.4,35.7,35.5,33.2,32.2$. HRMS (ASAP): Found $2 \mathrm{MH}^{+} 633.0999, \mathrm{C}_{34} \mathrm{H}_{45} \mathrm{O}_{2} \mathrm{Br}_{2}$ requires 633.0998 .

\section{4-Phenyltetrahydro-2H-thiopyran (53)}

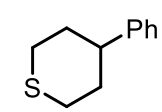

Following GP4 but using cumOOTES ( $80 \mathrm{mg}, 85 \mu \mathrm{L}, 0.30 \mathrm{mmol}, 3.0$ equiv.) and no TMHD, 4-iodotetrahydro-2 $H$-thiopyran $\mathbf{S 4}(22.8 \mathrm{mg}, 0.10 \mathrm{mmol})$ and boronate $\mathbf{2 a}(0.50 \mathrm{~mL}, 0.50 \mathrm{M}$ in THF, $0.25 \mathrm{mmol})$ gave $53(11.0 \mathrm{mg}, 62 \%)$ as an oil. ${ }^{1} \mathrm{H} \mathrm{NMR}\left(500 \mathrm{MHz}, \mathrm{CDCl}_{3}\right) \delta 7.33-7.28$ $(2 \mathrm{H}, \mathrm{m}), 7.23-7.17(3 \mathrm{H}, \mathrm{m}), 2.85(2 \mathrm{H}, \mathrm{ddd}, J=13.7,12.3,2.4 \mathrm{~Hz}), 2.73-2.68(2 \mathrm{H}, \mathrm{m}), 2.52$ $(1 \mathrm{H}, \mathrm{tt}, J=12.2,3.2 \mathrm{~Hz}), 2.19-2.10(2 \mathrm{H}, \mathrm{m}), 1.91-1.82(2 \mathrm{H}, \mathrm{m}) ;{ }^{13} \mathrm{C} \mathrm{NMR}\left(126 \mathrm{MHz}, \mathrm{CDCl}_{3}\right)$ $\delta$ 146.9, 128.5, 126.8, 126.3, 44.3, 35.1, 29.3. HRMS (ASAP): Found $\mathrm{MH}^{+}$179.0885, $\mathrm{C}_{11} \mathrm{H}_{15} \mathrm{SNa}$ requires 179.0889 .

\section{3-Phenyloxetane (54)}

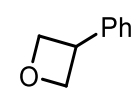

Following GP4 but using cumOOTES ( $80 \mathrm{mg}, 85 \mu \mathrm{L}, 0.30 \mathrm{mmol}, 3.0$ equiv.) and no TMHD, 3-iodooxetane $(18.4 \mathrm{mg}, 0.10 \mathrm{mmol})$ and boronate $\mathbf{2 a}(0.50 \mathrm{~mL}, 0.50 \mathrm{M}$ in THF, $0.25 \mathrm{mmol})$ gave 54 (10 mg, 74\%) as an oil. ${ }^{1} \mathrm{H}$ NMR (400 MHz, $\left.\mathrm{CDCl}_{3}\right) \delta$ 7.42-7.36 (4H, m), 7.30-7.27 $(1 \mathrm{H}, \mathrm{m}), 5.08(2 \mathrm{H}, \mathrm{dd}, J=8.4,6.0 \mathrm{~Hz}), 4.79(2 \mathrm{H}, \mathrm{dd}, \mathrm{J}=6.8,6.0 \mathrm{~Hz}), 4.29-4.19(1 \mathrm{H}, \mathrm{m})$. Data in accordance with the literature. ${ }^{31}$

\section{Diisopropyl 3-Phenylcyclobutane-1,1-dicarboxylate (55)}

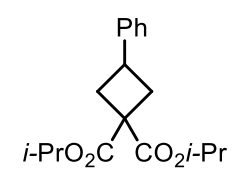

Following GP4 but using $\mathrm{Cu}(\mathrm{MeCN})_{4} \mathrm{PF}_{6}(11 \mathrm{mg}, 0.030 \mathrm{mmol}, 30 \mathrm{~mol} \%)$, cumOOTES (80 $\mathrm{mg}, 85 \mu \mathrm{L}, 0.30 \mathrm{mmol}, 3.0$ equiv.) and no TMHD, diisopropyl 3-iodocyclobutane-1,1- 
dicarboxylate $\mathbf{S 1}(35 \mathrm{mg}, 0.10 \mathrm{mmol})$ and boronate $\mathbf{2 a}(0.50 \mathrm{~mL}, 0.50 \mathrm{M}$ in THF, $0.25 \mathrm{mmol})$ gave $55(21 \mathrm{mg}, 68 \%)$ as an oil. ${ }^{1} \mathrm{H}$ NMR $\left(400 \mathrm{MHz}, \mathrm{CDCl}_{3}\right) \delta$ 7.34-7.28 (2H, m), 7.25-7.18 $(3 \mathrm{H}, \mathrm{m}), 5.15(1 \mathrm{H}$, hept, $J=6.3 \mathrm{~Hz}), 5.05(1 \mathrm{H}$, hept, $J=6.3 \mathrm{~Hz}), 3.61(1 \mathrm{H}, \mathrm{p}, J=9.3 \mathrm{~Hz}), 2.94$ $2.85(2 \mathrm{H}, \mathrm{m}), 2.71-2.62(2 \mathrm{H}, \mathrm{m}), 1.28(6 \mathrm{H}, \mathrm{d}, J=6.3 \mathrm{~Hz}), 1.24(6 \mathrm{H}, \mathrm{d}, J=6.3 \mathrm{~Hz}) .{ }^{13} \mathrm{C} \mathrm{NMR}$ $\left(101 \mathrm{MHz} \mathrm{CDCl}_{3}\right) \delta 171.5,170.9,144.2,128.4,126.5,126.4,68.9,68.7,49.2,36.1,33.8,21.6$, 21.5. HRMS (ASAP): Found $\mathrm{MH}^{+} 305.1741, \mathrm{C}_{18} \mathrm{H}_{25} \mathrm{O}_{4}$ requires 305.1747.

\section{4-Phenylcyclohexan-1-one (56)}

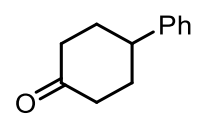

Following GP4 but using cumOOTES ( $80 \mathrm{mg}, 85 \mu \mathrm{L}, 0.30 \mathrm{mmol}, 3.0$ equiv.) and no TMHD, 4-iodocyclohexan-1-one $\mathbf{S 9}(22 \mathrm{mg}, 0.10 \mathrm{mmol})$ and boronate $\mathbf{2 a}(0.50 \mathrm{~mL}, 0.50 \mathrm{M}$ in THF, $0.25 \mathrm{mmol})$ gave $56(9 \mathrm{mg}, 53 \%)$ as an oil. ${ }^{1} \mathrm{H} \mathrm{NMR}\left(400 \mathrm{MHz}, \mathrm{CDCl}_{3}\right) \delta 7.36-7.29(2 \mathrm{H}, \mathrm{m})$, 7.28-7.20 (3H, m), $3.03(1 \mathrm{H}, \mathrm{tt}, \mathrm{J}=12.2,3.7 \mathrm{~Hz}), 2.58-2.44(4 \mathrm{H}, \mathrm{m}), 2.32-2.19(2 \mathrm{H}, \mathrm{m})$, $2.01-1.89(2 \mathrm{H}, \mathrm{m})$. Data in accordance with the literature. ${ }^{29}$

\section{8-Phenyl-1,4-dioxaspiro[4.5]decane (57)}

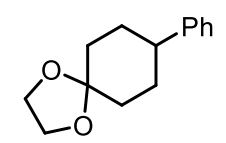

Following GP4 but using cumOOTES ( $80 \mathrm{mg}, 85 \mu \mathrm{L}, 0.30 \mathrm{mmol}, 3.0$ equiv.) and no TMHD, 8-iodo-1,4-dioxaspiro[4.5]decane $\mathbf{S 3}$ (27 mg, $0.10 \mathrm{mmol})$ and boronate 2a (0.50 mL, 0.50 M in THF, $0.25 \mathrm{mmol})$ gave $57(13 \mathrm{mg}, 61 \%)$ as an oil. ${ }^{1} \mathrm{H}$ NMR (400 $\left.\mathrm{MHz}, \mathrm{CDCl}_{3}\right) \delta 7.35-7.26$ (2H, m), 7.26-7.16 (3H, m), 3.99 (4H, s), 2.61-2.49 (1H, m), 1.91-1.84 (4H, m), 1.83-1.74 $(2 \mathrm{H}, \mathrm{m}), 1.74-1.65(2 \mathrm{H}, \mathrm{m})$. Data in accordance with the literature. ${ }^{29}$

\section{trans-2-Methoxycyclohexyl)benzene (58)}

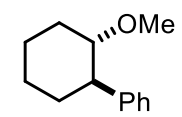

Following GP4 but using cumOOTES ( $80 \mathrm{mg}, 85 \mu \mathrm{L}, 0.30 \mathrm{mmol}, 3.0$ equiv.) and no TMHD, trans-1-iodo-2-methoxycyclohexane $\mathbf{S 1 3}(24.0 \mathrm{mg}, 0.10 \mathrm{mmol})$ and boronate $\mathbf{2 a}(0.50 \mathrm{~mL}, 0.50$ $\mathrm{M}$ in THF, $0.25 \mathrm{mmol})$ gave 58 (19 mg, 63\%) as an oil. ${ }^{1} \mathrm{H} \mathrm{NMR}\left(400 \mathrm{MHz}, \mathrm{CDCl}_{3}\right) \delta 7.37-$ $7.12(5 \mathrm{H}, \mathrm{m}), 3.27$ (1H, dt, $J=4.7,10.6 \mathrm{~Hz}), 3.10(3 \mathrm{H}, \mathrm{s}), 2.65-2.45(1 \mathrm{H}, \mathrm{m}), 2.32-2.20(1 \mathrm{H}$, $\mathrm{m}), 1.95-1.67(3 \mathrm{H}, \mathrm{m}), 1.60-1.15(4 \mathrm{H}, \mathrm{m})$. Data in accordance with the literature. ${ }^{32}$ 


\section{2-Phenyl-2,3-dihydro-1H-indene (59)}

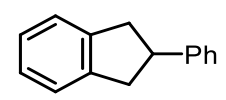

Following GP4 but using $\mathrm{Cu}(\mathrm{MeCN})_{4} \mathrm{PF}_{6}(11 \mathrm{mg}, 30 \% \mathrm{~mol})$, cumOOTES (80 mg, $85 \mu \mathrm{L}, 0.30$ mmol, 3.0 equiv.) and no TMHD, 2-iodo-2,3-dihydro- $1 H$-indene $\mathbf{S 8}$ (24 $\mathrm{mg}, 0.10 \mathrm{mmol})$ and boronate $2 \mathbf{a}(0.50 \mathrm{~mL}, 0.50 \mathrm{M}$ in THF, $0.25 \mathrm{mmol})$ gave $59(12 \mathrm{mg}, 64 \%)$ as an oil. ${ }^{1} \mathrm{H} \mathrm{NMR}$ $\left(400 \mathrm{MHz} \mathrm{CDCl}_{3}\right) \delta 7.30-7.24(4 \mathrm{H}, \mathrm{m}), 7.22-7.12(5 \mathrm{H}, \mathrm{m}), 3.65(1 \mathrm{H}, \mathrm{p}, \mathrm{J}=8.6 \mathrm{~Hz}), 3.31(2 \mathrm{H}$, $\mathrm{dd}, \mathrm{J}=15.5,8.2 \mathrm{~Hz}), 3.05(2 \mathrm{H}, \mathrm{dd}, \mathrm{J}=15.5,9.0 \mathrm{~Hz})$. Data in accordance with the literature. ${ }^{33}$

\section{Propane-1,2-diyldibenzene (60)}

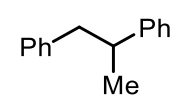

Following GP4 but using cumOOTES ( $80 \mathrm{mg}, 85 \mu \mathrm{L}, 0.30 \mathrm{mmol}, 3.0$ equiv.) and no TMHD, (2-iodopropyl)benzene $\mathbf{S 2}(24.6 \mathrm{mg}, 0.10 \mathrm{mmol})$ and boronate $\mathbf{2 a}(0.50 \mathrm{~mL}, 0.50 \mathrm{M}$ in THF, $0.25 \mathrm{mmol})$ gave $60(17 \mathrm{mg}, 73 \%)$ as an oil. ${ }^{1} \mathrm{H}$ NMR (400 MHz, $\left.\mathrm{CDCl}_{3}\right) \delta 7.34-7.15(8 \mathrm{H}, \mathrm{m})$, 7.13-7.07 (2H, m), 3.08-2.93 (2H, m), 2.83-2.75 (1H, m), $1.26(3 \mathrm{H}, \mathrm{d}, J=6.8 \mathrm{~Hz})$. Data in accordance with the literature. ${ }^{34}$

\section{4-Phenyl-1-oxaspiro[5.5] undecane (61)}

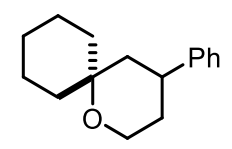

Following GP4 but using cumOOTES ( $80 \mathrm{mg}, 85 \mu \mathrm{L}, 0.30 \mathrm{mmol}, 3.0$ equiv.) and no TMHD, 4-iodo-1-oxaspiro[5.5] undecane $\mathbf{S 1 5}$ (28.0 mg, $0.10 \mathrm{mmol})$ and boronate $2 \mathbf{a}(0.50 \mathrm{~mL}, 0.50 \mathrm{M}$ in THF, $0.25 \mathrm{mmol})$ gave 61 (14 mg, 73\%) as an oil. ${ }^{1} \mathrm{H} \mathrm{NMR}\left(400 \mathrm{MHz}, \mathrm{CDCl}_{3}\right) \delta 7.33-7.28$ $(2 \mathrm{H}, \mathrm{m}), 7.23-7.18(3 \mathrm{H}, \mathrm{m}), 3.85-3.73(1 \mathrm{H}, \mathrm{m}), 2.97-2.90(1 \mathrm{H}, \mathrm{m}), 2.14-2.10(1 \mathrm{H}, \mathrm{m}), 1.80-$ $1.26(14 \mathrm{H}, \mathrm{m})$. Data in accordance with the literature. ${ }^{35}$

tert-Butyl 2-Phenyl-7-azaspiro[3.5]nonane-7-carboxylate (62)

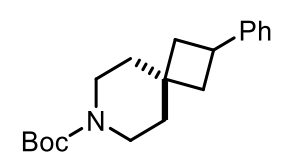

Following GP4 but using cumOOTES ( $80 \mathrm{mg}, 85 \mu \mathrm{L}, 0.30 \mathrm{mmol}, 3.0$ equiv.) and no TMHD, tert-butyl 2-iodo-7-azaspiro[3.5]nonane-7-carboxylate $\mathbf{S 6}(35.1 \mathrm{mg}, 0.10 \mathrm{mmol})$ and boronate 2a $(0.50 \mathrm{~mL}, 0.50 \mathrm{M}$ in THF, $0.25 \mathrm{mmol})$ gave $62(6 \mathrm{mg}, \mathrm{XX} \%)$ as an oil. ${ }^{1} \mathrm{H} \mathrm{NMR}(400 \mathrm{MHz}$, $\left.\mathrm{CDCl}_{3}\right) \delta 7.36-7.26(2 \mathrm{H}, \mathrm{m}), 7.24-7.14(3 \mathrm{H}, \mathrm{m}), 3.52(1 \mathrm{H}, \mathrm{p}, J=9.1 \mathrm{~Hz}), 3.45-3.38(2 \mathrm{H}, \mathrm{m})$, 
3.34-3.25 (2H, m), 2.35-2.24 (2H, m), 1.95-1.85 (2H, m), 1.74-1.68 (2H, m), 1.54-1.48 (2H, $\mathrm{m}), 1.47(9 \mathrm{H}, \mathrm{s})$. Data in accordance with the literature. ${ }^{36}$

tert-Butyl 6-Phenyl-2-azaspiro[3.3]heptane-2-carboxylate (63)

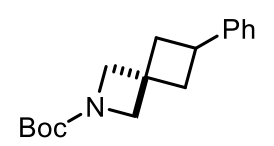

Following GP4, but using cumOOTES ( $80 \mathrm{mg}, 85 \mu \mathrm{L}, 0.30 \mathrm{mmol}, 3.0$ equiv.) and no TMHD, tert-butyl 6-iodo-2-azaspiro[3.3] heptane-2-carboxylate $\mathbf{S 5}$ (32 $\mathrm{mg}, 0.10 \mathrm{mmol})$ and boronate 2a $(0.50 \mathrm{~mL}, 0.50 \mathrm{M}$ in THF, $0.25 \mathrm{mmol})$ gave $63(18 \mathrm{mg}, 65 \%)$ as an oil. ${ }^{1} \mathrm{H} \mathrm{NMR}(500 \mathrm{MHz}$, $\left.\mathrm{CDCl}_{3}\right) \delta 7.34-7.28(2 \mathrm{H}, \mathrm{m}), 7.22-7.14(3 \mathrm{H}, \mathrm{m}), 4.06(2 \mathrm{H}, \mathrm{s}), 3.84(2 \mathrm{H}, \mathrm{s}), 3.39(1 \mathrm{H}, \mathrm{p}, J=8.9$ $\mathrm{Hz}), 2.62-2.53(2 \mathrm{H}, \mathrm{m}), 2.32-2.25(2 \mathrm{H}, \mathrm{m}), 1.44(9 \mathrm{H}, \mathrm{s}) .{ }^{13} \mathrm{C} \mathrm{NMR}\left(126 \mathrm{MHz}, \mathrm{CDCl}_{3}\right) \delta 156.3$, 144.6, 128.3, 126.2, 126.1, 79.3, 62.2 (br.), 60.2 (br.), 40.2, 34.1, 33.9, 28.4. HRMS (ESI): Found $\mathrm{MNa}^{+} 296.1612, \mathrm{C}_{17} \mathrm{H}_{23} \mathrm{O}_{2} \mathrm{NNa}$ requires 296.1621 .

tert-Butyl 6-(3-Fluoro-4-methoxyphenyl)-2-azaspiro[3.3]heptane-2-carboxylate (64)

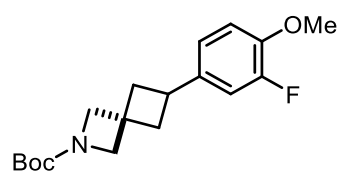

Following GP4 but using cumOOTES ( $80 \mathrm{mg}, 85 \mu \mathrm{L}, 0.30 \mathrm{mmol}, 3.0$ equiv.) and no TMHD, iodide $\mathbf{S 5}(32 \mathrm{mg}, 0.10 \mathrm{mmol})$, boronate $2 \operatorname{ar}(0.50 \mathrm{~mL}, 0.50 \mathrm{M}$ in THF, $0.25 \mathrm{mmol})$ gave 64 (30 mg, 94\%) as an oil. ${ }^{1} \mathrm{H}$ NMR (400 MHz, $\left.\mathrm{CDCl}_{3}\right) \delta 6.92-6.82(3 \mathrm{H}, \mathrm{m}), 4.04(2 \mathrm{H}, \mathrm{s}), 3.86$ (3H, s), 3.83 (2H, s), 3.38-3.22 (1H, m), 2.62-2.44 (2H, m), 2.25-2.16 (2H, m), $1.44(9 \mathrm{H}, \mathrm{s})$; ${ }^{13} \mathrm{C} \mathrm{NMR}\left(101 \mathrm{MHz}, \mathrm{CDCl}_{3}\right) \delta 156.4,152.5(\mathrm{~d}, J=245.8 \mathrm{~Hz}), 146.0(\mathrm{~d}, J=10.8 \mathrm{~Hz}), 138.1(\mathrm{~d}$, $J=5.6 \mathrm{~Hz}), 121.9(\mathrm{~d}, J=3.4 \mathrm{~Hz}), 114.2(\mathrm{~d}, J=18.0 \mathrm{~Hz}), 113.5(\mathrm{~d}, J=2.2 \mathrm{~Hz}), 79.5,62.3,60.3$, 56.6, 40.5, 34.0, 33.5, 28.6; ${ }^{19} \mathrm{~F} \mathrm{NMR}\left(376 \mathrm{MHz}, \mathrm{CDCl}_{3}\right) \delta-135.3$. HRMS (ESI) found $\mathrm{MNa}^{+}$ 344.1632, $\mathrm{C}_{18} \mathrm{H}_{24} \mathrm{O}_{3} \mathrm{NFNa}$ requires 344.1632 .

tert-Butyl 6-(4-(Trifluoromethoxy)phenyl)-2-azaspiro[3.3]heptane-2-carboxylate (65)

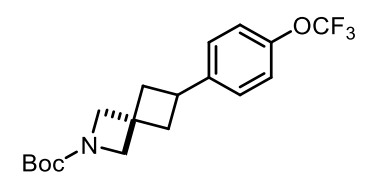

Following GP4 but using cumOOTES ( $80 \mathrm{mg}, 85 \mu \mathrm{L}, 0.30 \mathrm{mmol}, 3.0$ equiv.) and no TMHD, iodide S5 (32 mg, $0.10 \mathrm{mmol})$, boronate $2 \mathbf{k}(0.50 \mathrm{~mL}, 0.50 \mathrm{M}$ in THF, $0.25 \mathrm{mmol})$ gave $\mathbf{6 5}$ (29 $\mathrm{mg}, 81 \%)$ as an oil. ${ }^{1} \mathrm{H}$ NMR $\left(500 \mathrm{MHz}, \mathrm{CDCl}_{3}\right) \delta 7.17(2 \mathrm{H}, \mathrm{d}, J=8.6 \mathrm{~Hz}), 7.13(2 \mathrm{H}, \mathrm{d}, J=$ 
$8.4 \mathrm{~Hz}), 4.06(2 \mathrm{H}, \mathrm{s}), 3.84(2 \mathrm{H}, \mathrm{s}), 3.39(1 \mathrm{H}, \mathrm{t}, J=8.9 \mathrm{~Hz}), 2.64-2.53(2 \mathrm{H}, \mathrm{m}), 2.29-2.21(2 \mathrm{H}$, m), $1.44(9 \mathrm{H}, \mathrm{s}) ;{ }^{13} \mathrm{C} \mathrm{NMR}\left(126 \mathrm{MHz}, \mathrm{CDCl}_{3}\right) \delta 156.4,147.6(\mathrm{~d}, J=1.9 \mathrm{~Hz}), 143.5,127.7$, 121.1, 120.6 (q, $J=256.6 \mathrm{~Hz}), 79.5,62.3,60.3,40.4,34.0,33.7,28.6 ;{ }^{19} \mathrm{~F}$ NMR $(376 \mathrm{MHz}$, $\left.\mathrm{CDCl}_{3}\right) \delta-58.0$; HRMS (ESI) found: $\mathrm{MNa}^{+} 380.1433, \mathrm{C}_{18} \mathrm{H}_{22} \mathrm{O}_{3} \mathrm{NF}_{3} \mathrm{Na}$ requires 380.1444.

\section{1,3-Dimethyl-7-(2-phenylpropyl)-3,7-dihydro-1H-purine-2,6-dione (68)}

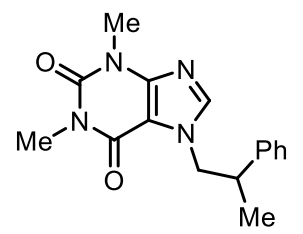

Following GP4 but using cumOOTES ( $80 \mathrm{mg}, 85 \mu \mathrm{L}, 0.30 \mathrm{mmol}, 3.0$ equiv.) and no TMHD, 7-(2-iodopropyl)-1,3-dimethyl-3,7-dihydro-1H-purine-2,6-dione $\mathbf{S 1 2}$ (35 mg, $0.10 \mathrm{mmol}$ ) and boronate $2 \mathbf{a}(0.50 \mathrm{~mL}, 0.50 \mathrm{M}$ in THF, $0.25 \mathrm{mmol})$ gave $\mathbf{6 8}(16 \mathrm{mg}, 53 \%)$ as an oil. ${ }^{1} \mathrm{H} \mathrm{NMR}$ $\left(400 \mathrm{MHz}, \mathrm{CDCl}_{3}\right) \delta$ 7.33-7.27 (2H, m), 7.26-7.20 (1H, m), 7.16-7.11 (2H, m), 7.04 (1H, s), $4.48(1 \mathrm{H}, \mathrm{dd}, J=13.4,6.6 \mathrm{~Hz}), 4.25(1 \mathrm{H}, \mathrm{dd}, J=13.4,8.5 \mathrm{~Hz}), 3.56(3 \mathrm{H}, \mathrm{s}), 3.44(3 \mathrm{H}, \mathrm{s}), 3.44-$ $3.29(1 \mathrm{H}, \mathrm{m}), 1.35(3 \mathrm{H}, \mathrm{d}, J=7.0 \mathrm{~Hz}) .{ }^{13} \mathrm{C} \mathrm{NMR}\left(101 \mathrm{MHz}, \mathrm{CDCl}_{3}\right) \delta 155.3,151.7,148.8$, 142.2, 141.2, 128.8, 127.3, 127.0, 106.7, 54.3, 40.9, 29.8, 28.0, 18.0. HRMS (ASAP): Found $\mathrm{MH}^{+} 299.1491, \mathrm{C}_{16} \mathrm{H}_{19} \mathrm{O}_{2} \mathrm{~N}_{4}$ requires 299.1503.

\section{1,3-Dimethyl-7-(2-(1-methyl-1H-pyrazol-4-yl)propyl)-3,7-dihydro-1H-purine-2,6-dione} (69)

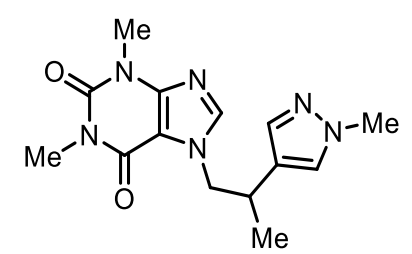

Following GP4 but using cumOOTES ( $80 \mathrm{mg}, 85 \mu \mathrm{L}, 0.30 \mathrm{mmol}, 3.0$ equiv.) and no TMHD, 7-(2-iodopropyl)-1,3-dimethyl-3,7-dihydro-1H-purine-2,6-dione $\mathbf{S 1 2}$ (35 $\mathrm{mg}, 0.10 \mathrm{mmol}$ ) and boronate $2 \mathbf{z}(0.50 \mathrm{~mL}, 0.50 \mathrm{M}$ in THF, $0.25 \mathrm{mmol})$ gave $69(11 \mathrm{mg}, 35 \%)$ as an oil. ${ }^{1} \mathrm{H} \mathrm{NMR}$ $\left(400 \mathrm{MHz}, \mathrm{CDCl}_{3}\right) \delta 7.28(1 \mathrm{H}, \mathrm{s}), 7.27(1 \mathrm{H}, \mathrm{s}), 7.09(1 \mathrm{H}, \mathrm{s}), 4.32(1 \mathrm{H}, \mathrm{dd}, J=13.3,7.0 \mathrm{~Hz})$, $4.23(1 \mathrm{H}, \mathrm{dd}, J=13.3,7.7 \mathrm{~Hz}), 3.84(3 \mathrm{H}, \mathrm{s}), 3.59(3 \mathrm{H}, \mathrm{s}), 3.43(3 \mathrm{H}, \mathrm{s}), 3.33(1 \mathrm{H}, \mathrm{h}, J=7.1 \mathrm{~Hz})$, $1.28(3 \mathrm{H}, \mathrm{d}, J=7.0 \mathrm{~Hz}) .{ }^{13} \mathrm{C}$ NMR $\left(101 \mathrm{MHz}, \mathrm{CDCl}_{3}\right) \delta 155.2,151.7,148.9,141.3,137.1$, 127.8, 122.8, 106.8, 54.4, 38.9, 31.4, 29.8, 28.0, 18.2. HRMS (ASAP): Found $\mathrm{MH}^{+} 303.1561$, $\mathrm{C}_{14} \mathrm{H}_{19} \mathrm{O}_{2} \mathrm{~N}_{6}$ requires 303.1564 . 


\section{(E)-7-(5-((tert-Butyldimethylsilyl)oxy)-2-methylpent-3-en-1-yl)-1,3-dimethyl-3,7-}

\section{dihydro-1H-purine-2,6-dione (70)}

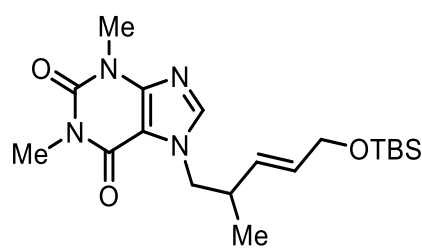

Following GP4 but using cumOOTES ( $80 \mathrm{mg}, 85 \mu \mathrm{L}, 0.30 \mathrm{mmol}, 3.0$ equiv.) and no TMHD, 7-(2-iodopropyl)-1,3-dimethyl-3,7-dihydro-1H-purine-2,6-dione S12 (35 $\mathrm{mg}, 0.10 \mathrm{mmol}$ ) and boronate $2 \mathrm{aj}(0.50 \mathrm{~mL}, 0.50 \mathrm{M}$ in THF, $0.25 \mathrm{mmol})$ gave $70(16 \mathrm{mg}, 42 \%)$ as an oil. ${ }^{1} \mathrm{H} \mathrm{NMR}$ $\left(400 \mathrm{MHz}, \mathrm{CDCl}_{3}\right) \delta 7.45(1 \mathrm{H}, \mathrm{s}), 5.57-5.37(2 \mathrm{H}, \mathrm{m}), 4.23(1 \mathrm{H}, \mathrm{dd}, J=13.4,6.5 \mathrm{~Hz}), 4.13-$ $4.03(3 \mathrm{H}, \mathrm{m}), 3.58(3 \mathrm{H}, \mathrm{s}), 3.41(3 \mathrm{H}, \mathrm{s}), 2.86-2.72(1 \mathrm{H}, \mathrm{m}), 1.06(3 \mathrm{H}, \mathrm{d}, J=6.8 \mathrm{~Hz}), 0.88(9 \mathrm{H}$, s), $0.03(6 \mathrm{H}, \mathrm{s}) .{ }^{13} \mathrm{C} \mathrm{NMR}\left(101 \mathrm{MHz}, \mathrm{CDCl}_{3}\right) \delta 155.2,151.7,148.9,141.3,131.5,130.6,106.9$, 63.2, 52.6, 37.8, 29.8, 28.0, 25.9, 18.4, 17.4, -5.3. HRMS (ASAP): Found $\mathrm{MH}^{+}$393.2310, $\mathrm{C}_{19} \mathrm{H}_{33} \mathrm{O}_{3} \mathrm{~N}_{4} \mathrm{Si}$ requires 393.2316.

\section{4,5-cis-3-Benzylhexahydro-4H-furo[2,3-b]pyran (72)}

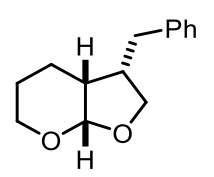

Following GP4 but using cumOOTES ( $80 \mathrm{mg}, 85 \mu \mathrm{L}, 0.30 \mathrm{mmol}, 3.0$ equiv.) and no TMHD, 2-(allyloxy)-3-iodotetrahydro-2H-pyran $\mathbf{S 1 4}(26.8 \mathrm{mg}, 0.10 \mathrm{mmol})$ and boronate $\mathbf{2 a}(0.50 \mathrm{~mL}$, $0.50 \mathrm{M}$ in THF, $0.25 \mathrm{mmol})$ gave $72(12 \mathrm{mg}$, d.r. $12: 1,53 \%)$ as an oil. ${ }^{1} \mathrm{H}$ NMR (400 MHz, $\left.\mathrm{CDCl}_{3}\right) \delta 7.29(2 \mathrm{H}, \mathrm{t}, J=7.4 \mathrm{~Hz}), 7.20(2 \mathrm{H}, \mathrm{t}, J=7.5 \mathrm{~Hz}), 7.17(1 \mathrm{H}, \mathrm{d}, J=7.2 \mathrm{~Hz}), 5.28(1 \mathrm{H}$, d, $J=3.7 \mathrm{~Hz}), 3.88(1 \mathrm{H}, \mathrm{t}, J=7.7 \mathrm{~Hz}), 3.78(2 \mathrm{H}, \mathrm{t}, J=8.7 \mathrm{~Hz}), 3.65(1 \mathrm{H}, \mathrm{d}, J=11.3 \mathrm{~Hz}), 2.78-$ $2.59(3 \mathrm{H}, \mathrm{m}), 2.00-1.91(1 \mathrm{H}, \mathrm{m}), 1.81-1.72(1 \mathrm{H}, \mathrm{m}), 1.66-1.51(3 \mathrm{H}, \mathrm{m})$. Data in accordance with the literature. ${ }^{37}$

\section{3-Benzyl-1-tosylpyrrolidine (74)}

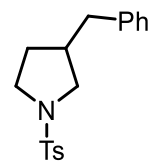

Following GP4 but using cumOOTES ( $80 \mathrm{mg}, 85 \mu \mathrm{L}, 0.30 \mathrm{mmol}, 3.0$ equiv.) and no TMHD, $N$-allyl- $N$-(2-iodoethyl)-4-methylbenzenesulfonamide S11 (36.5 mg, $0.10 \mathrm{mmol}$ ) and boronate 2a $(0.50 \mathrm{~mL}, 0.50 \mathrm{M}$ in THF, $0.25 \mathrm{mmol})$ gave $74(15 \mathrm{mg}, 48 \%)$ as an oil. ${ }^{1} \mathrm{H}$ NMR (400 MHz, $\left.\mathrm{CDCl}_{3}\right) \delta 7.70(2 \mathrm{H}, \mathrm{d}, J=8.2 \mathrm{~Hz}), 7.32(2 \mathrm{H}, \mathrm{d}, J=7.9 \mathrm{~Hz}), 7.29-7.16(3 \mathrm{H}, \mathrm{m}), 7.05(2 \mathrm{H}, \mathrm{d}, J=$ 
$6.8 \mathrm{~Hz}), 3.42-3.31(2 \mathrm{H}, \mathrm{m}), 3.19(1 \mathrm{H}, \mathrm{dt}, J=9.2,8.0 \mathrm{~Hz}), 2.91(1 \mathrm{H}, \mathrm{dd}, J=9.9,7.5 \mathrm{~Hz}), 2.54$ $(2 \mathrm{H}, \mathrm{d}, J=7.7 \mathrm{~Hz}), 2.44(3 \mathrm{H}, \mathrm{s}), 2.37-2.27(1 \mathrm{H}, \mathrm{m}), 1.90-1.84(1 \mathrm{H}, \mathrm{m}), 1.55-1.46(1 \mathrm{H}, \mathrm{m})$. Data in accordance with the literature. ${ }^{38}$ 


\section{Mechanistic Studies}

\subsection{Proposed Mechanism}

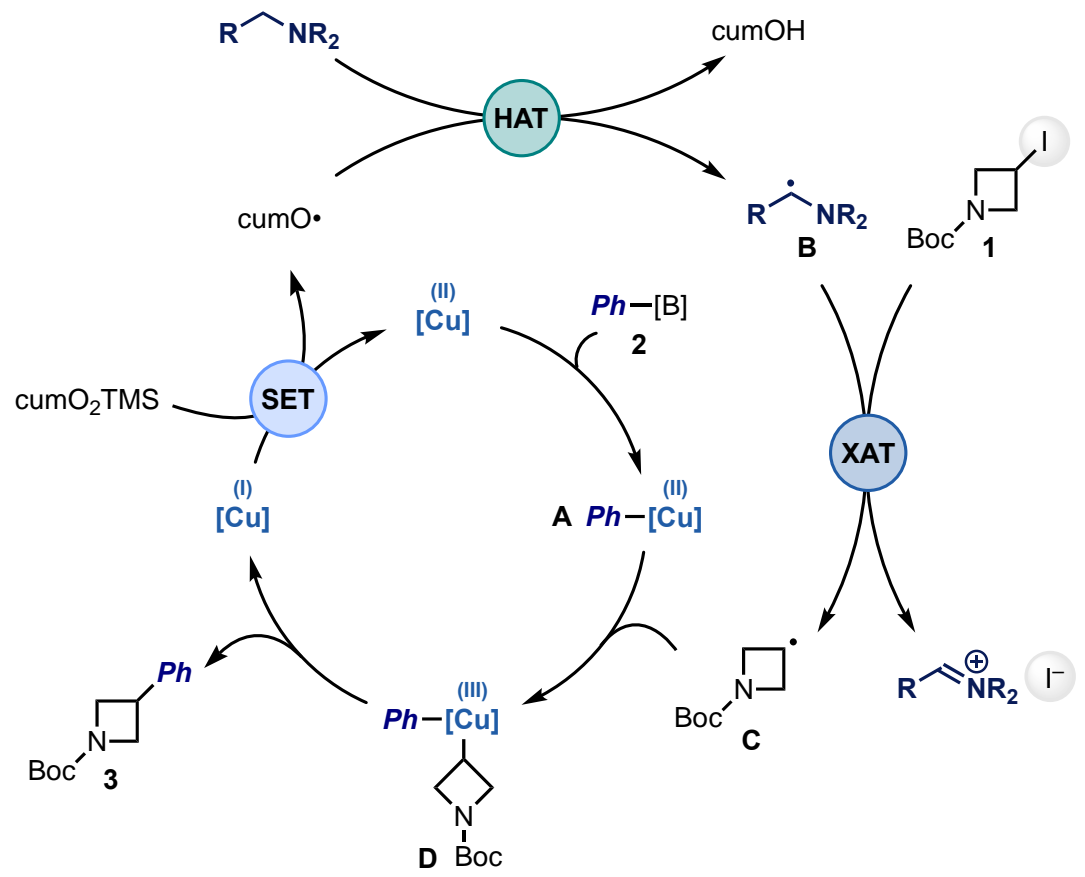

Scheme S4.

\subsubsection{Probing the Oxidation of $[\mathrm{Cu}(\mathrm{I})]$}

The oxidation of $[\mathrm{Cu}(\mathrm{I})]$ to $[\mathrm{Cu}(\mathrm{II})]$ by cumO ${ }_{2} \mathrm{TMS}$ was demonstrated by UV/Vis absorption spectroscopy studies showing the formation of a new band $(\lambda=750 \mathrm{~nm})$, which is characteristic for $[\mathrm{Cu}(\mathrm{II})]$ species.

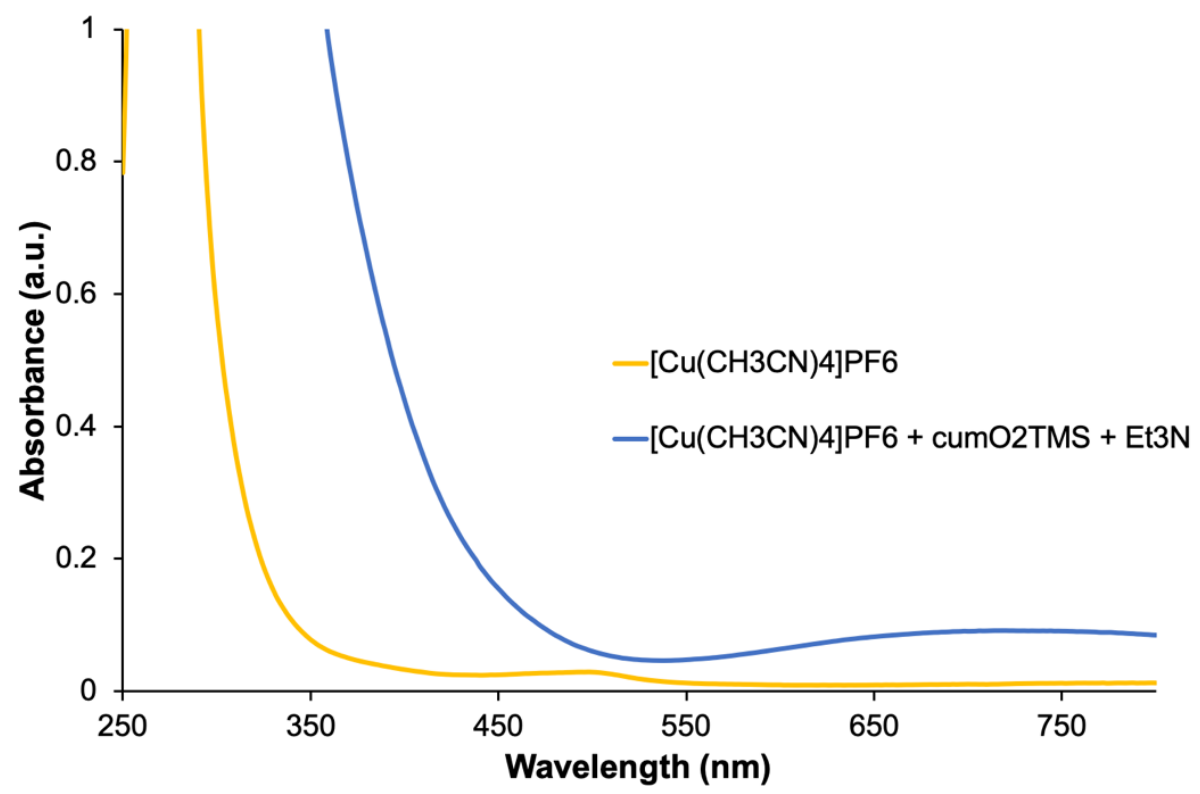

Figure S1. 


\subsubsection{Probing the Transmetalation of $2 \mathrm{a}$ with $[\mathrm{Cu}(\mathrm{II})]$}

The transmetalation of $\mathbf{2 a}$ with $[\mathrm{Cu}(\mathrm{II})]$ was supported by UV/Vis absorption spectroscopy studies (Figure S2).

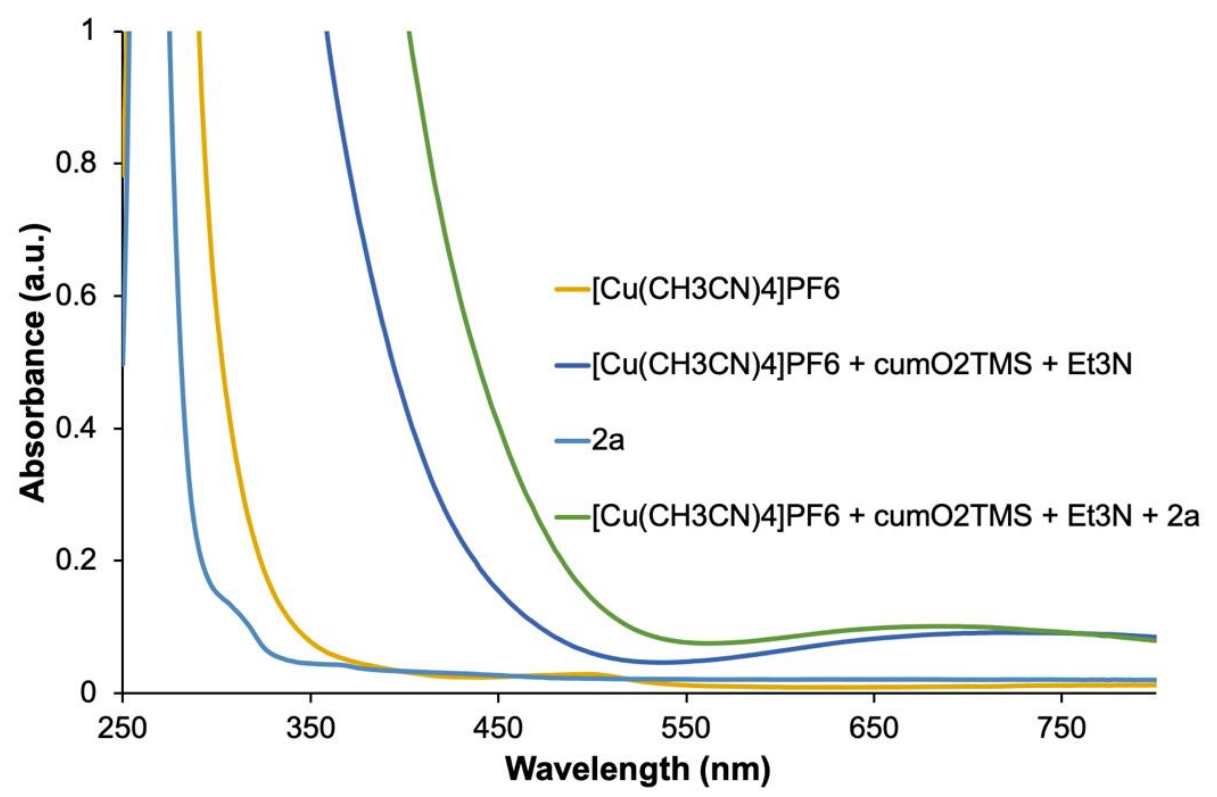

Figure S2.

The transmetalation between $2 \mathbf{a}$ and $[\mathrm{Cu}$ (II)] should lead to the formation of $\mathrm{Bu}-\mathrm{B}$ (pin), which was supported by ${ }^{11}$ B NMR spectroscopy studies and GC-MS analysis (Figure S3).

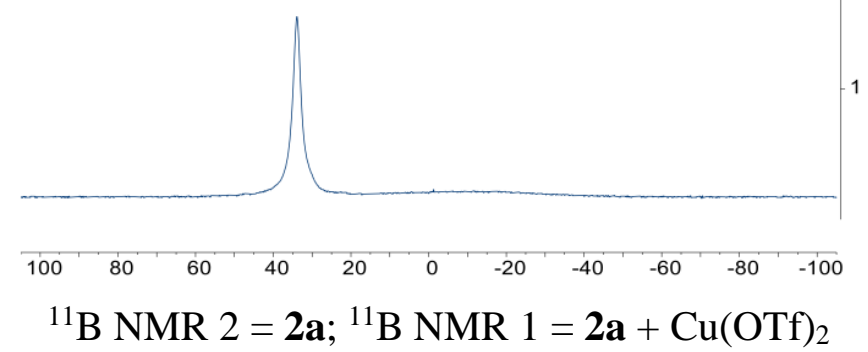

Figure S3.

Furthermore, a stoichiometric reaction between $\mathbf{2 a}$ and $[\mathrm{Cu}(\mathrm{II})]$ followed by water work up gave $\mathrm{Bu}-\mathrm{B}$ (pin) (Scheme S5). 


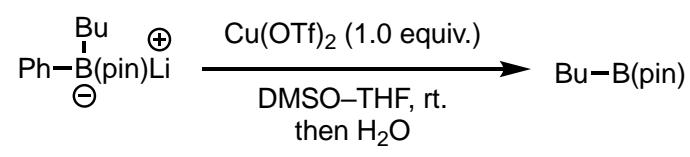

Scheme S5.

In order to obtain initial information regarding the lack of reactivity of organoborons $\mathbf{2} \mathbf{b}-\mathbf{d}$, we have evaluated the direct transmetalation of these species with $[\mathrm{Cu}(\mathrm{II})]$ by UV/VIS absorption spectroscopy (Figure S4). These studies indicates that these species might not be able to react with $[\mathrm{Cu}(\mathrm{II})]$ under our reaction conditions.

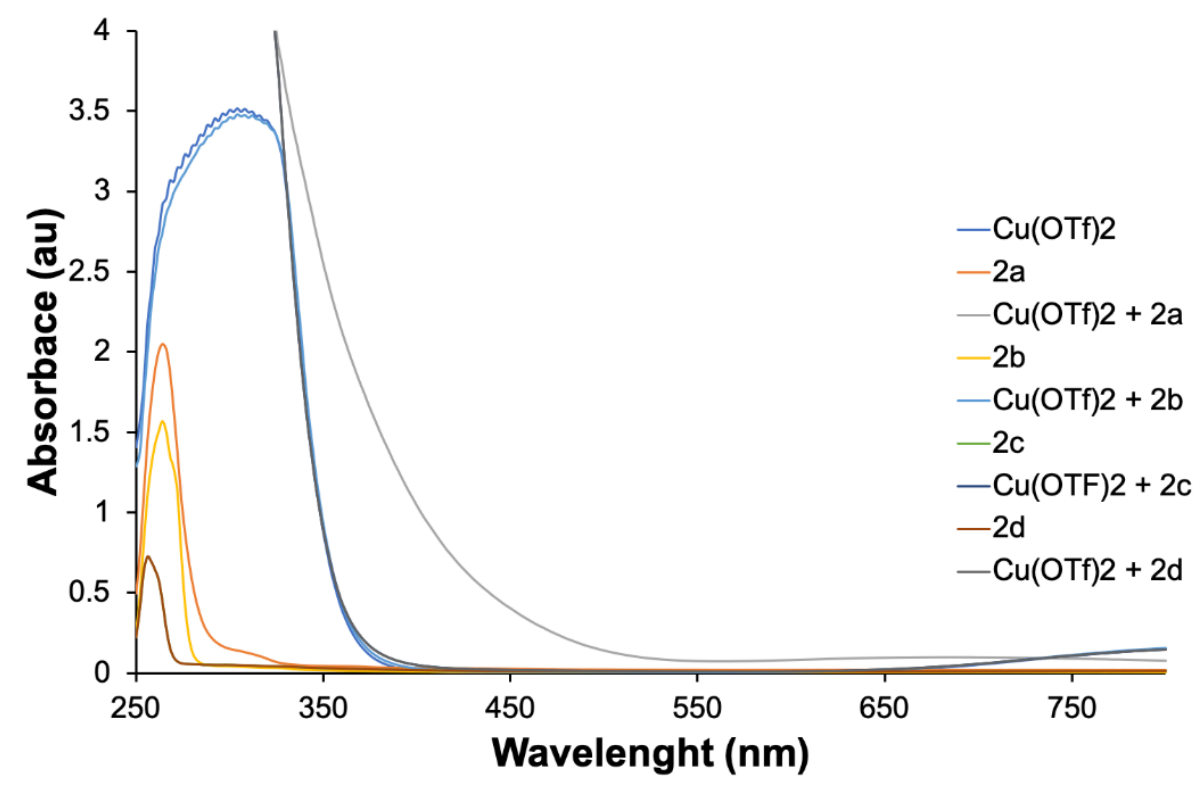

Figure S4.

\subsubsection{SET Oxidation of 2a}

Boronate complexes are electron rich species which can be oxidised. A ground state SET between 2a and cumO 2 TMS should be difficult (Equation S1).

$$
\begin{gathered}
\Delta G^{\mathrm{o}}=F\left(E_{\text {ox }}-E_{\text {red }}\right) \\
\Delta G^{\mathrm{o}}=F(0.37 \mathrm{~V}-(-1.61 \mathrm{~V}))=F(1.98 \mathrm{~V})
\end{gathered}
$$

Equation S1. 
Furthermore, 2a proved stable in the presence of $\mathrm{cumO}_{2} \mathrm{TMS}$ on the NMR time scale as demonstrated by ${ }^{11}$ B NMR spectroscopy studies (Figure S5). However, we cannot exclude that over time a SET might take place.

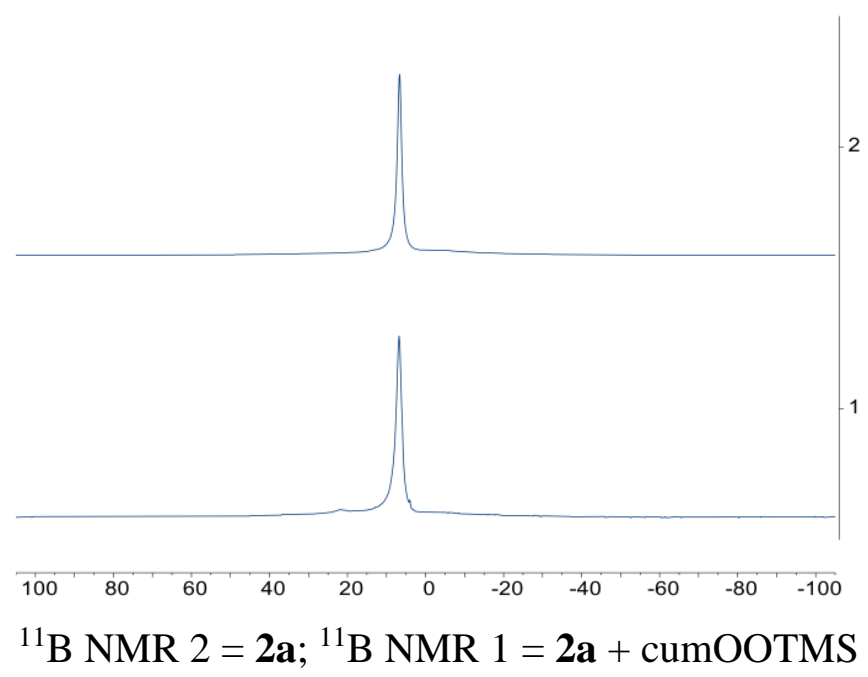

Figure S5.

\subsubsection{Probing $\alpha$-Aminoalkyl Radical Generation by HAT}

According to our proposed mechanism, the key $\alpha$-aminoalkyl radical $\mathbf{B}$ is generated by HAT from the cumO- on $\mathrm{Et}_{3} \mathrm{~N}$. To obtain more information on this step, we run competition experiments adding equimolar amount Hantzsch ester to the optimised reaction between $\mathbf{1}$ and 2a (Scheme S7). As Hantzsch ester has significantly weaker $\mathrm{C}\left(s p^{3}\right)-\mathrm{H}$ bonds than $\mathrm{Et}_{3} \mathrm{~N}$, it ought to undergo more facile HAT from cumO•. This should hamper $\alpha$-aminoalkyl radical generation and therefore product formation.

As shown in Scheme S6, upon addition of Hantzsch ester the reactivity was completely suppressed and $\mathbf{3}$ was not observed. 

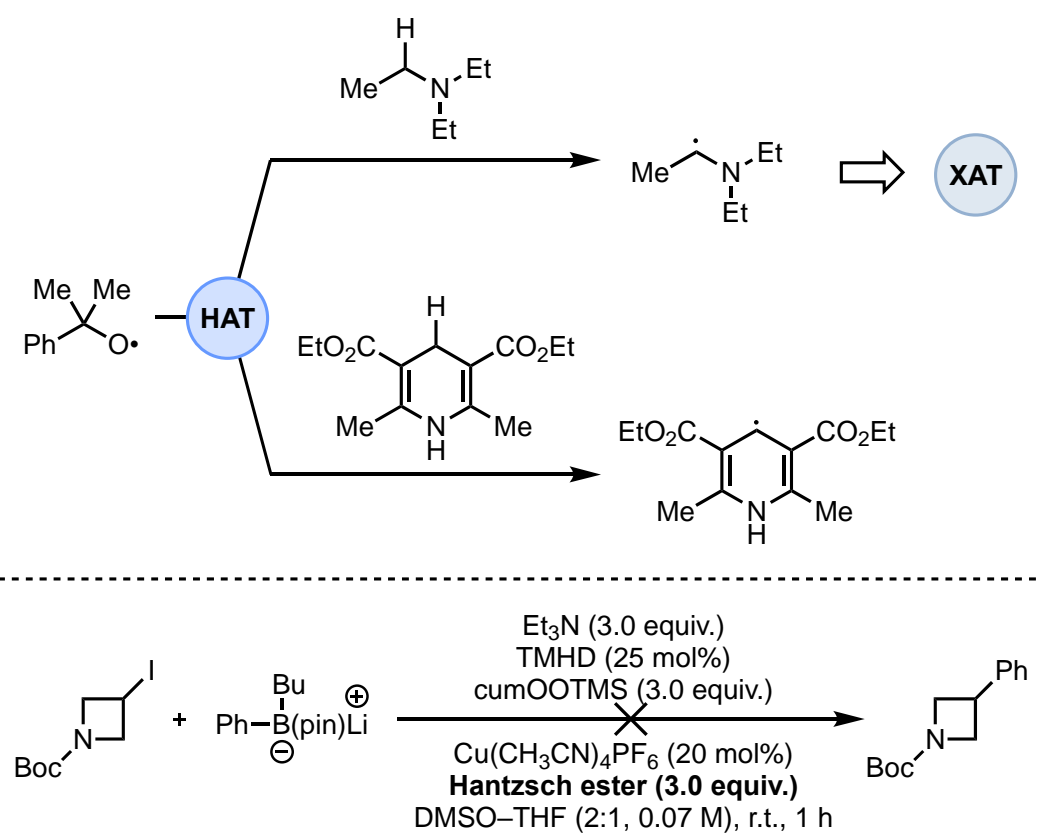

Scheme S6.

\subsubsection{Probing $\alpha$-Aminoalkyl Radical-Mediated XAT}

To confirm the generation of $\alpha$-aminoalkyl radicals and their involvement in the formation of the alkyl radical via XAT, we run the standard reaction between $\mathbf{1}$ and $\mathbf{2 a}$ but replacing $\mathrm{Et}_{3} \mathrm{~N}$ with other amines that cannot lead to the formation of $\alpha$-aminoalkyl radicals (Scheme S7).

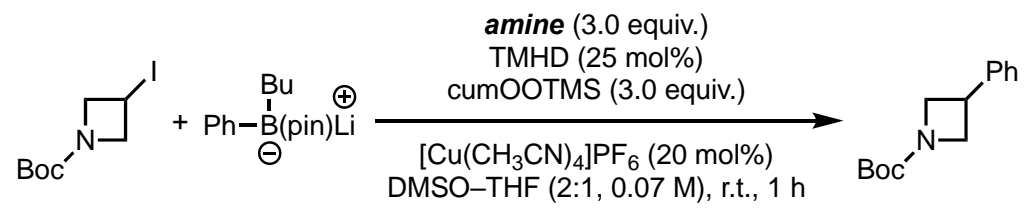

Scheme S7.

As shown in Table S4, the use of TMP and DABCO lead to formation of $\mathbf{3}$ in yield analogous to the experiment run in the absence of amine (compare entries 1 and 2 with 3 ). These results support the fact that with these two amines no $\alpha$-aminoalkyl radical is generated and therefore low yielding reactivity might occur via the formation of either $\mathrm{Ph} \bullet$ or $\mathrm{Me}$. 
Table S4.

\begin{tabular}{|c|c|c|c|}
\hline Entry & Amine & Yield (\%) & $\mathbf{1}(\%)$ \\
\hline $\mathbf{1}$ & \multirow{2}{2}{} & 19 & 76 \\
\hline $\mathbf{3}$ & - & 17 & 80 \\
\hline
\end{tabular}

\subsubsection{Ruling Out Direct SET Reduction of the Alkyl Iodide}

Unactivated secondary alkyl iodides have a low reduction potential $\left(E_{\text {red }}<-2 \mathrm{~V}\right.$ vs SCE$)$ that makes a direct SET with $[\mathrm{Cu}(\mathrm{I})]$ challenging. To support this hypothesis, we have attempted the reaction of $\mathbf{1}$ and $\mathbf{2 a}$ using a stoichiometric $[\mathrm{Cu}(\mathrm{I})]$. This experiment was performed by premixing $\mathbf{2 a}$ with the $[\mathrm{Cu}(\mathrm{I})]$, followed by the addition of $\mathbf{1}$.

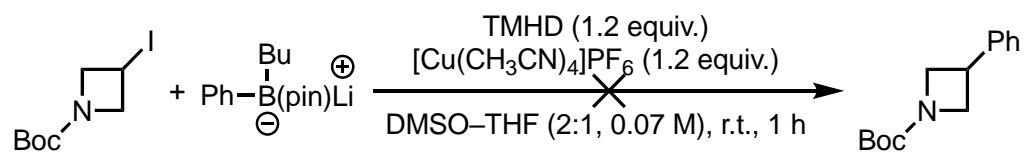

Scheme S8.

The fact that $\mathbf{3}$ was not formed and $\mathbf{1}$ was recovered in quantitative yield, confirm the inability of $[\mathrm{Cu}(\mathrm{I})]$ to undergo ground-state SET with $\mathbf{2 a}$ as well as direct oxidative addition.

\subsubsection{Probing Radical Capture by [Cu(II)]-Ar Species as C $\left(s p^{3}\right)-C\left(s p^{2}\right)$ Bond Forming Step}

To obtain more information supporting the capture of the alkyl radical $\mathbf{C}$ by the $\mathrm{Ph}-[\mathrm{Cu}(\mathrm{II})]$ species $\mathbf{A}$ to give a $\mathrm{Ph}$,alkyl-[Cu(III)] intermediated $\mathbf{D}$ followed by reductive elimination to $\mathbf{3}$, we studied the reaction between a pre-formed $[\mathrm{Cu}(\mathrm{II})]-\mathrm{Ph}$ complex [prepared by premixing $\mathbf{2 a}$ with $\mathrm{Cu}(\mathrm{OTf})_{2}$ ] with lauroyl peroxide 4 (Scheme S9). As 4 undergoes thermal O-O bond homolysis, ${ }^{[24]}$ the alkyl radical formation does not require SET or XAT activation. The desired product 5 was observed in $38 \%$ yield and this supports our proposed pathway for $\mathrm{C}\left(s p^{3}\right)-\mathrm{C}\left(s p^{2}\right)$ bond assembly. 


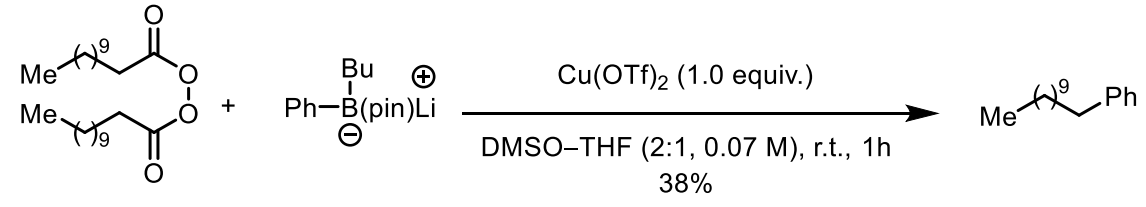

Scheme S9 


\subsection{Alternative Mechanism}

We also considered an alternative mechanism based on transmetalation of $\mathbf{2 a}$ onto $[\mathrm{Cu}(\mathrm{I})]$ followed by oxidation to [Cu(II)]-Ph (Scheme S10). However, we have not been able to obtain mechanistic evidence on the key group state SET (see below).

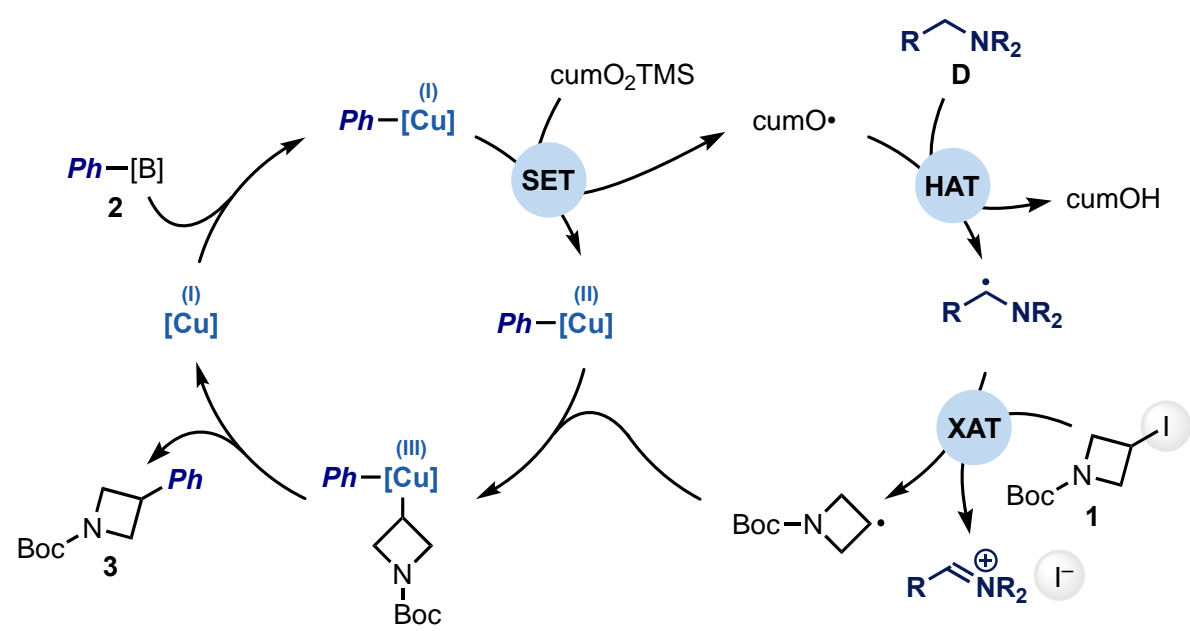

Scheme S10.

\subsubsection{Coordination of 2 a to $[\mathrm{Cu}(\mathrm{I})]$ to $\mathrm{Give}[\mathrm{Cu}(\mathrm{I})]-\mathrm{Ph}$}

In this proposed mechanism, the boronate 2a undergoes transmetalation with $[\mathrm{Cu}(\mathrm{I})]$, forming the $\mathrm{Ph}-[\mathrm{Cu}(\mathrm{I})]$ complex $\mathbf{A}$. We have evaluated the transmetalation by UV/Vis absorption spectroscopy and observed a strong change in the absorption profile (Figure S6).

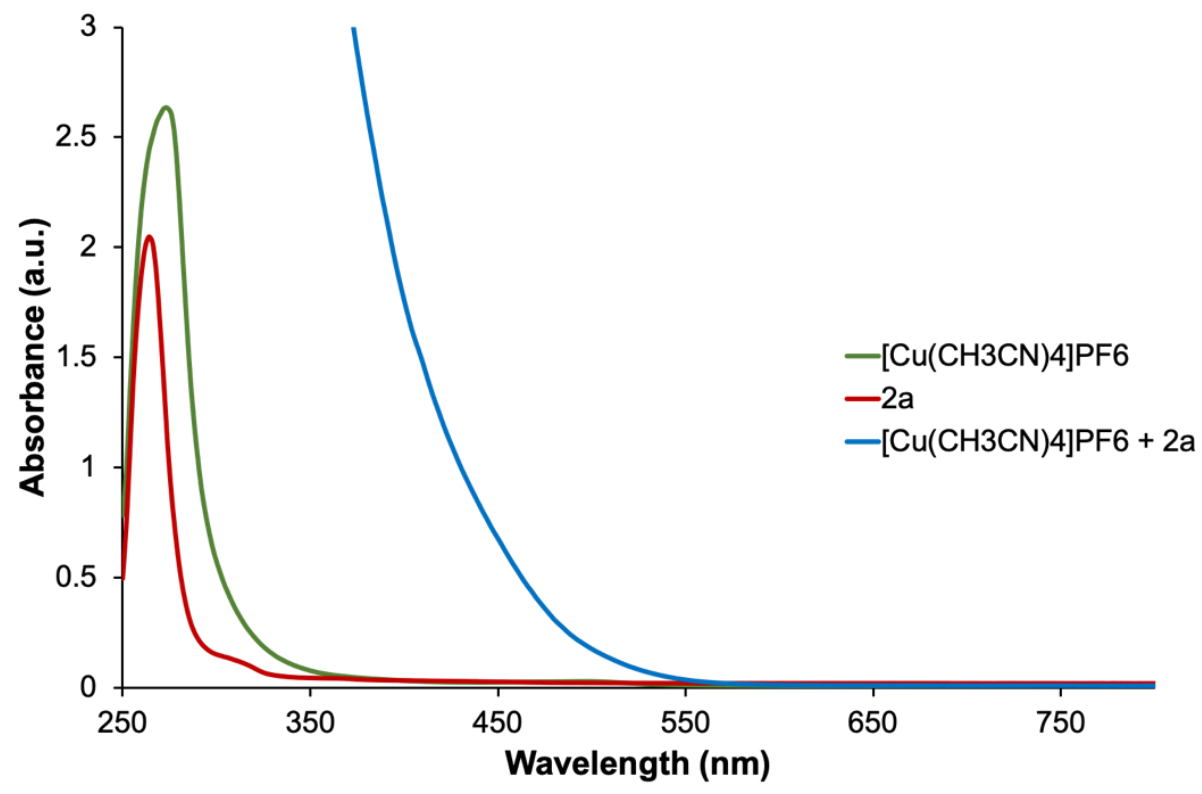

Figure S6. 


\subsubsection{Oxidation of $[\mathrm{Cu}(\mathrm{I})]-\mathrm{Ph}$ by cumO2 $\mathrm{TMS}$ to $[\mathrm{Cu}(\mathrm{II})]-\mathrm{Ph}$}

We have tried to follow the reaction of $\mathbf{A}$ with cumOOTMS but we did not succeed in observing

the formation of a $[\mathrm{Cu}(\mathrm{II})]$ species which should display a characteristic absorption at $750 \mathrm{~nm}$ by UV/Vis absorption spectroscopy analysis. 


\section{Cyclic Voltammetry Studies}

Cyclic voltammetry was conducted on an EmStat (PalmSens) potentiostat using a 3-electrodes cell configuration. A glassy carbon working electrode was employed alongside a platinum wire counter electrode and a $\mathrm{Ag} / \mathrm{AgCl}$ reference electrode. Solution was degassed by bubbling $\mathrm{N}_{2}$ prior to measurements. $5 \mathrm{mM}$ solution of the the boronate $\mathbf{2 a}$ was freshly prepared in dry acetonitrile along with $0.1 \mathrm{M}$ of tetrabutylammonium hexafluorophosphate as supporting electrolyte and was examined at a scan rate of $0.1 \mathrm{Vs}^{-1}$. Decamethylferrocene $\left(E_{1 / 2}=-0.125 \mathrm{~V}\right.$ vs SCE) was added at the end of the measurement as an internal standard to determine the precise potential scale. ${ }^{39}$ Potential values are given versus the saturated calomel electrode (SCE). Irreversible wave was obtained; therefore, the potentials was estimated at half the maximum the maximum current, as previously in the literature (Figure S7). ${ }^{40}$

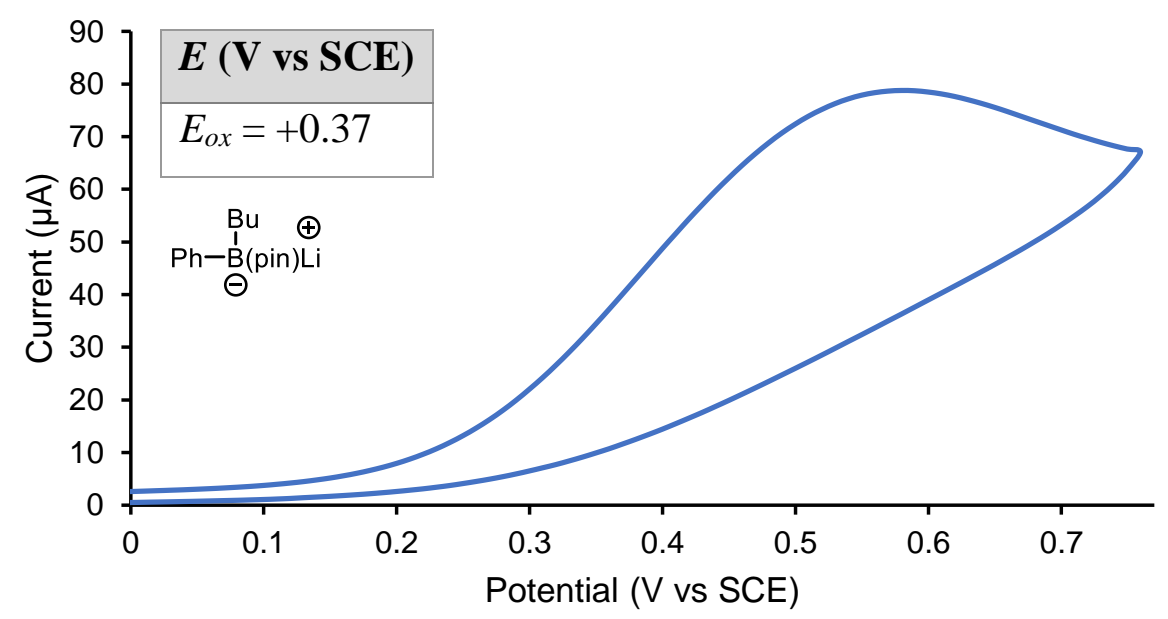

Figure S7. 


\section{NMR Spectra}

S1 - ${ }^{1} \mathrm{H}$ NMR (400 MHz, $\left.\mathrm{CDCl}_{3}\right)$

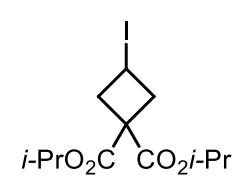

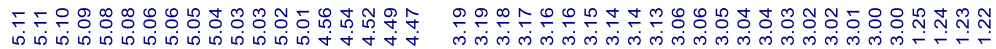

i- $-\mathrm{PrO}_{2} \mathrm{C} \quad \mathrm{CO}_{2} \mathrm{i}-\mathrm{Pr}$

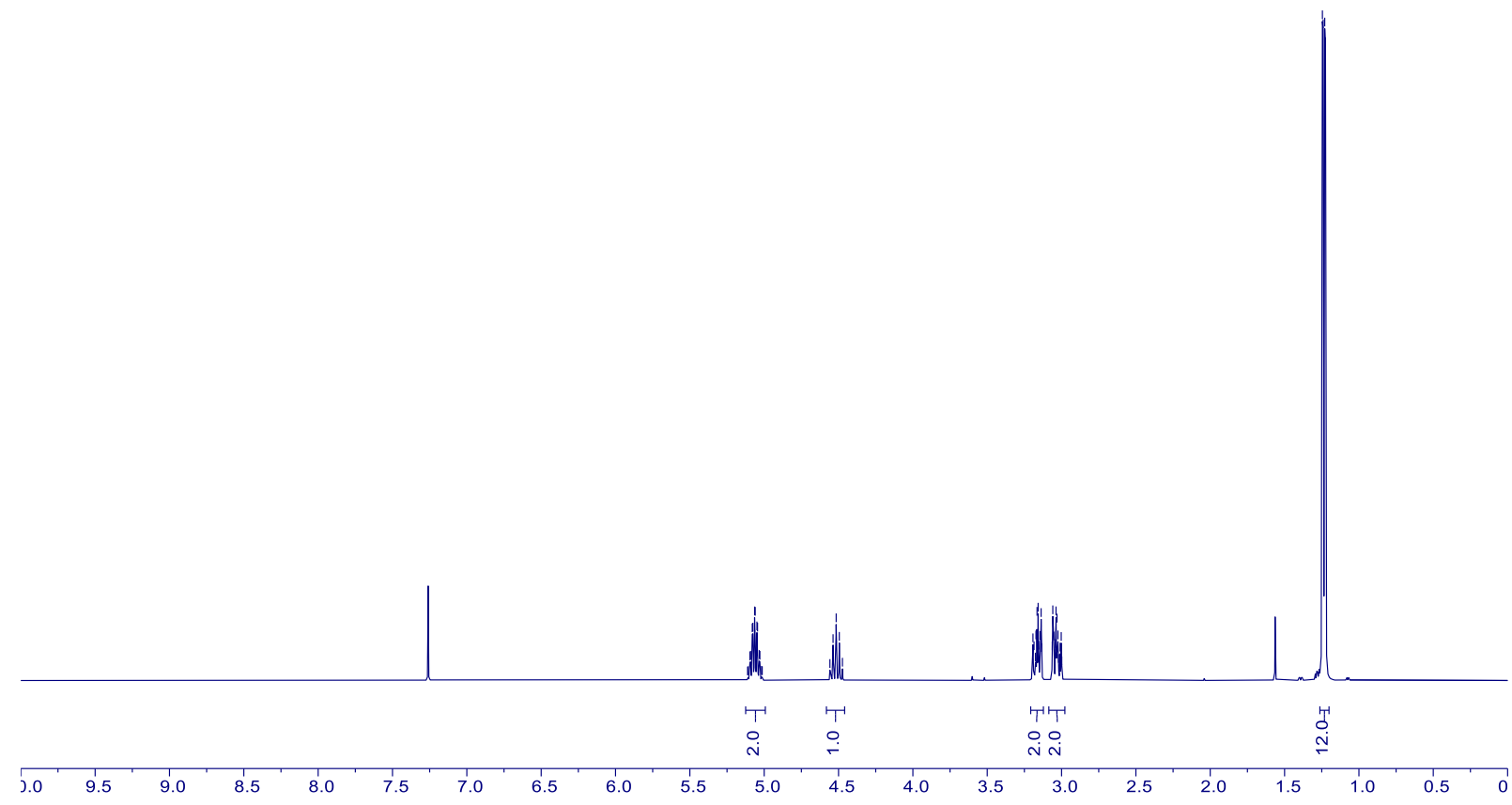

S1 $-{ }^{13} \mathrm{C}$ NMR $\left(101 \mathrm{MHz}, \mathrm{CDCl}_{3}\right)$

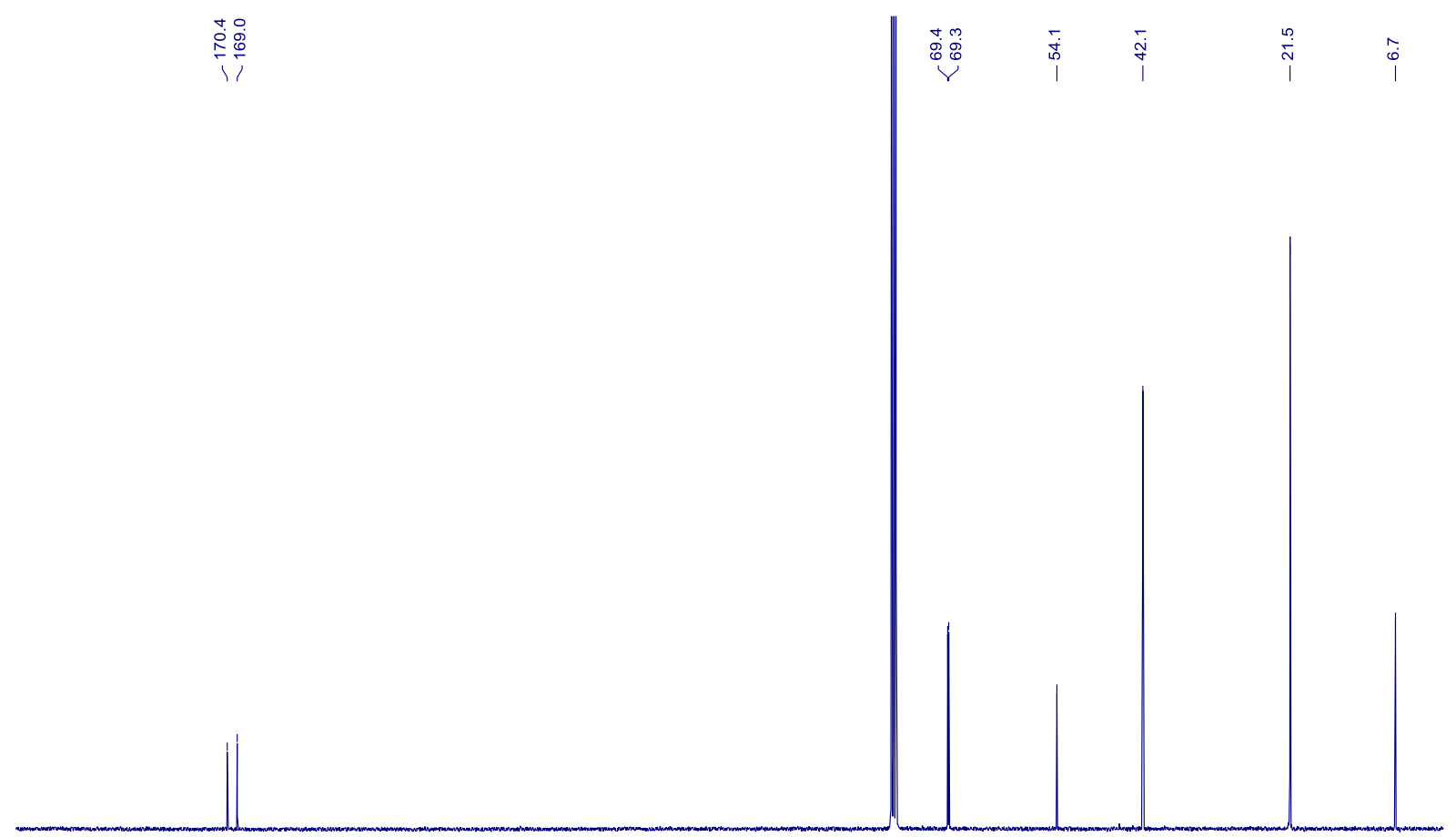

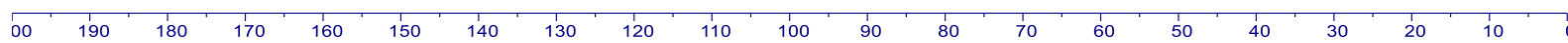


S17 - ${ }^{1} \mathrm{H}$ NMR $\left(400 \mathrm{MHz}, \mathrm{CDCl}_{3}\right)$

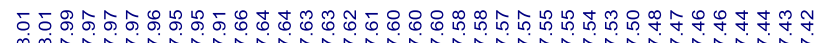

$\underbrace{\mathrm{Me}}_{\mathrm{O}-{ }^{-}} \mathrm{SiPh}_{2} t-\mathrm{Bu}$

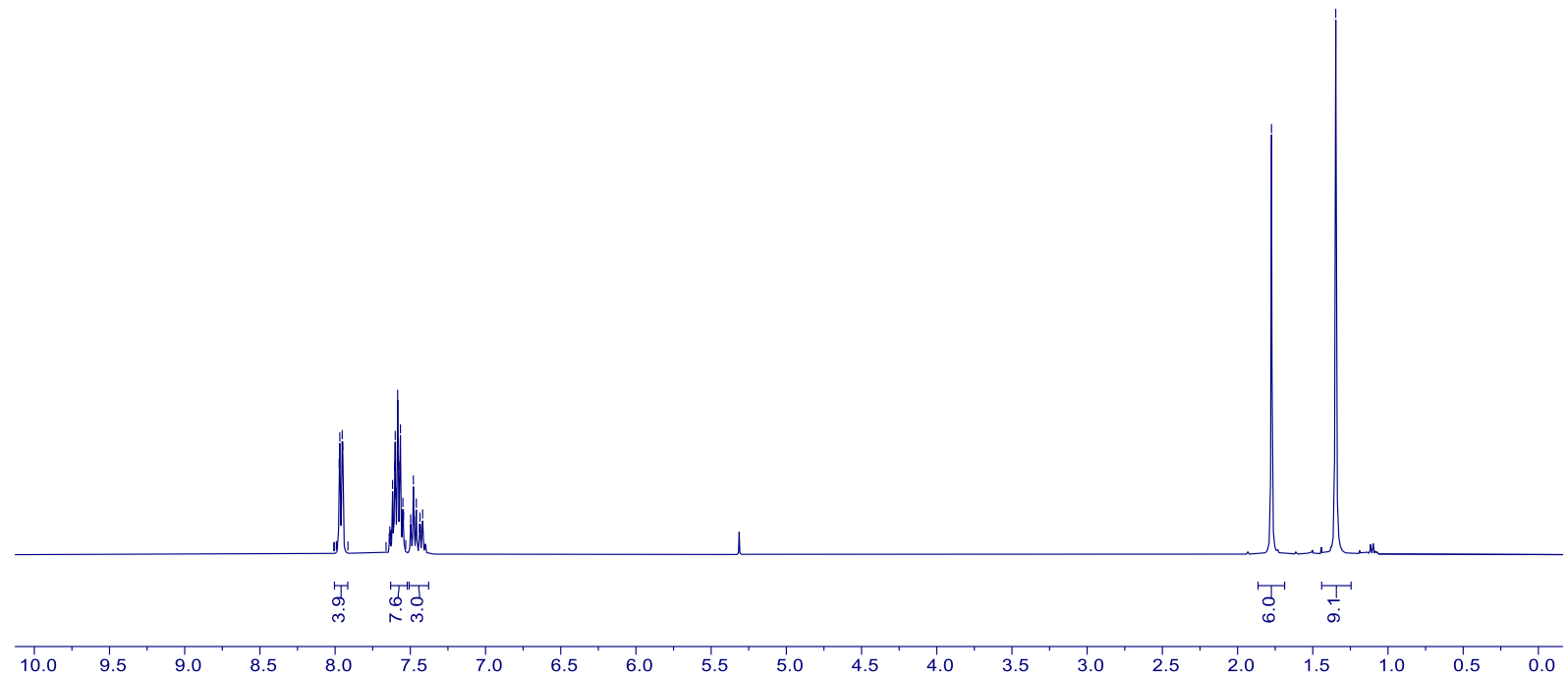

S17 - ${ }^{13} \mathrm{C}$ NMR (101 MHz, $\left.\mathrm{CDCl}_{3}\right)$
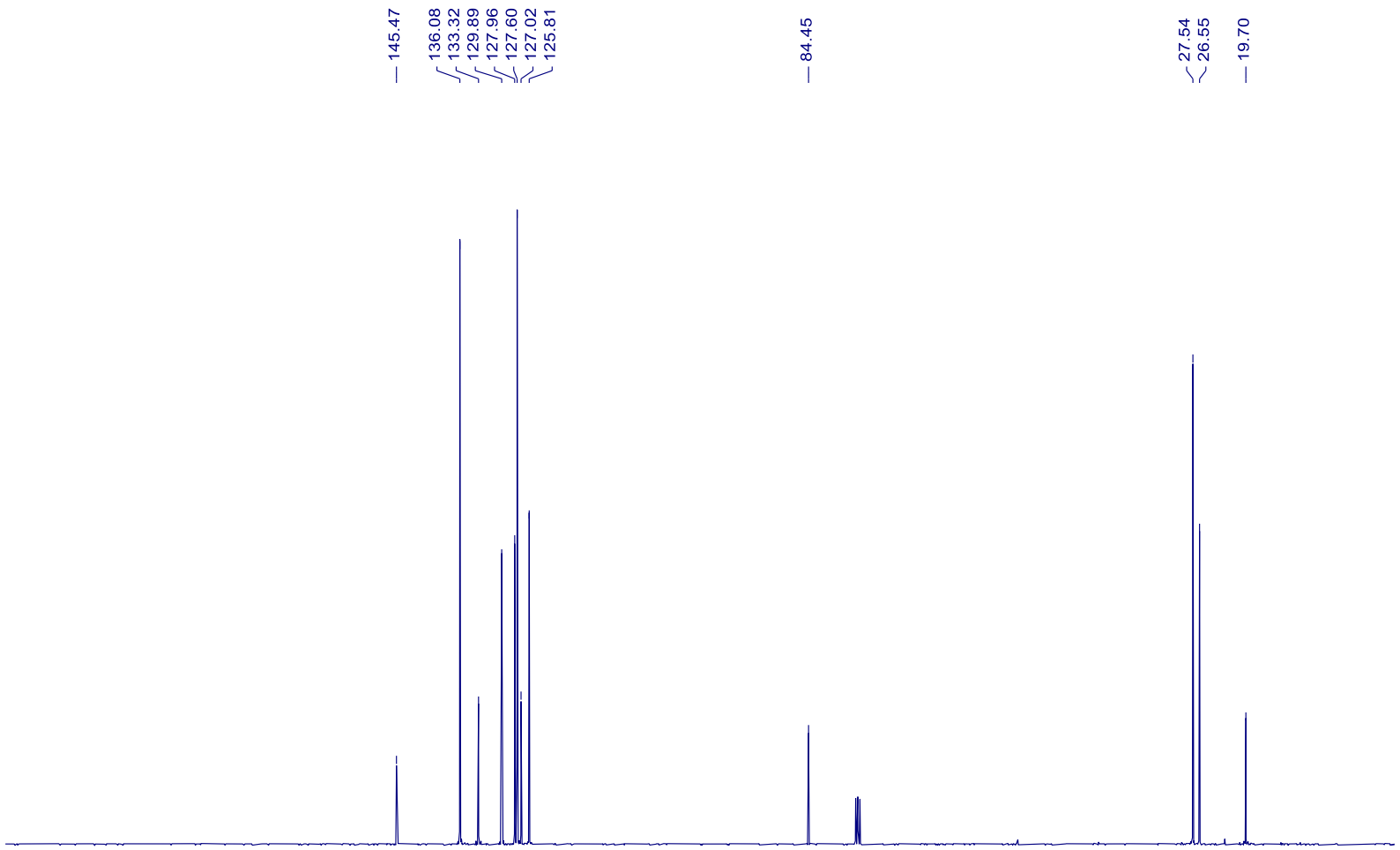

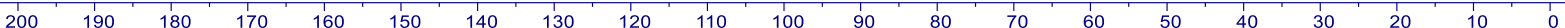


8- ${ }^{1} \mathrm{H}$ NMR (400 MHz, $\left.\mathrm{CDCl}_{3}\right)$<smiles>CCOc1ccc(C2CN(C(=O)c3ccccc3)C2)cc1</smiles>

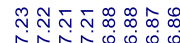

$8-{ }^{13} \mathrm{C} \mathrm{NMR}\left(101 \mathrm{MHz}, \mathrm{CDCl}_{3}\right)$

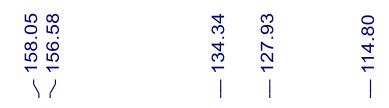

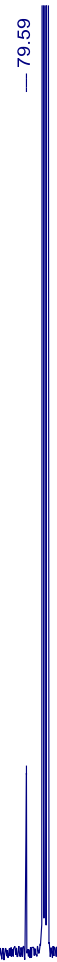

$\begin{array}{ccc}0 & 0 \\ 0 & 0 & 0 \\ 0 & 0 & 0 \\ 0 & \infty & 0 \\ 1 & 1 & 1\end{array}$ 
16 - ${ }^{1} \mathrm{H} \mathrm{NMR}\left(500 \mathrm{MHz}, \mathrm{CDCl}_{3}\right)$

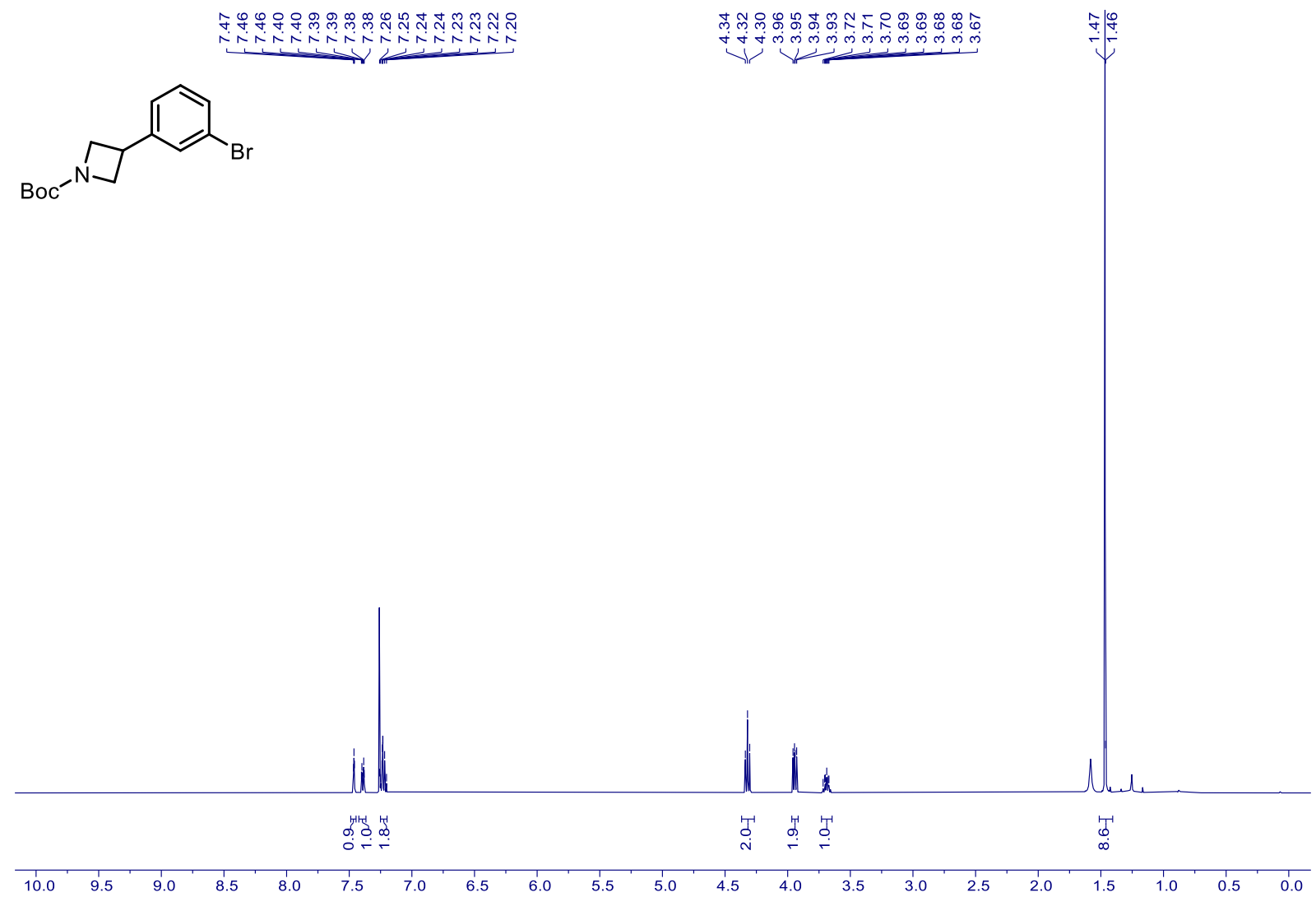

$16-{ }^{13} \mathrm{C}$ NMR $\left(126 \mathrm{MHz}, \mathrm{CDCl}_{3}\right)$

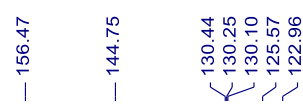

$\begin{array}{lll}9 & \stackrel{0}{0} & 0 \\ 0 & \infty \\ 0 & \infty & \infty \\ 0 & 1 & i\end{array}$

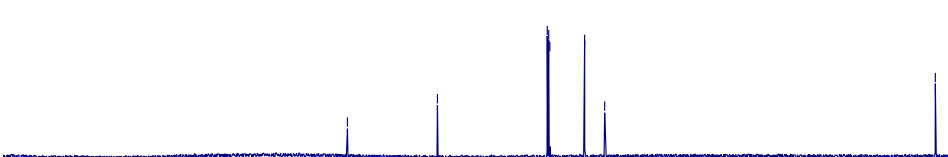

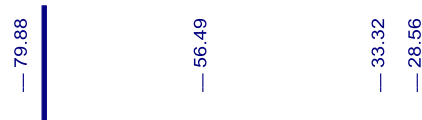

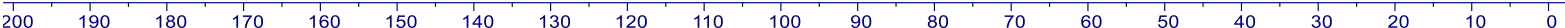


19 - ${ }^{1} \mathrm{H}$ NMR $\left(500 \mathrm{MHz}, \mathrm{CDCl}_{3}\right)$

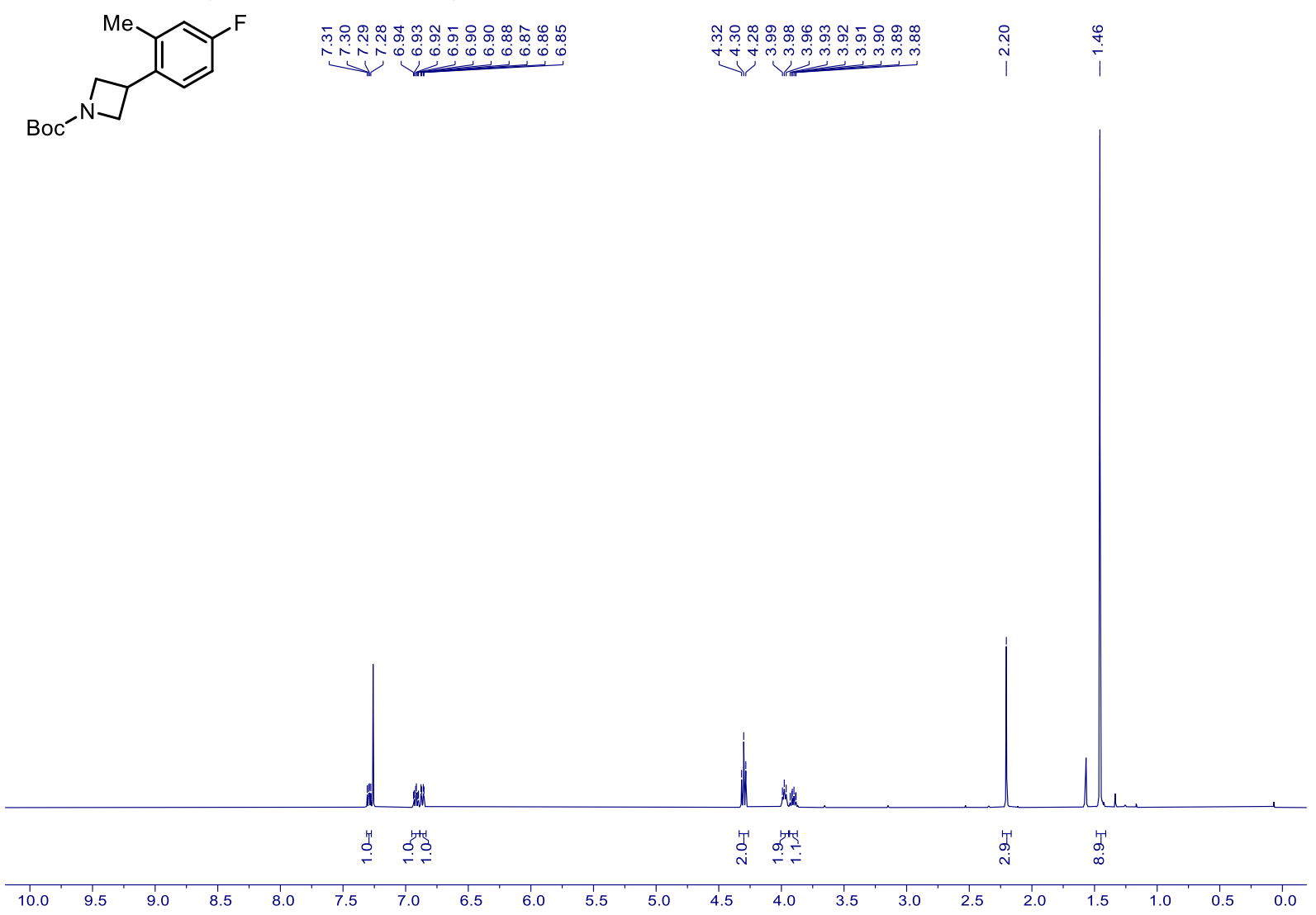

$19-{ }^{13} \mathrm{C} \mathrm{NMR}\left(126 \mathrm{MHz}, \mathrm{CDCl}_{3}\right)$

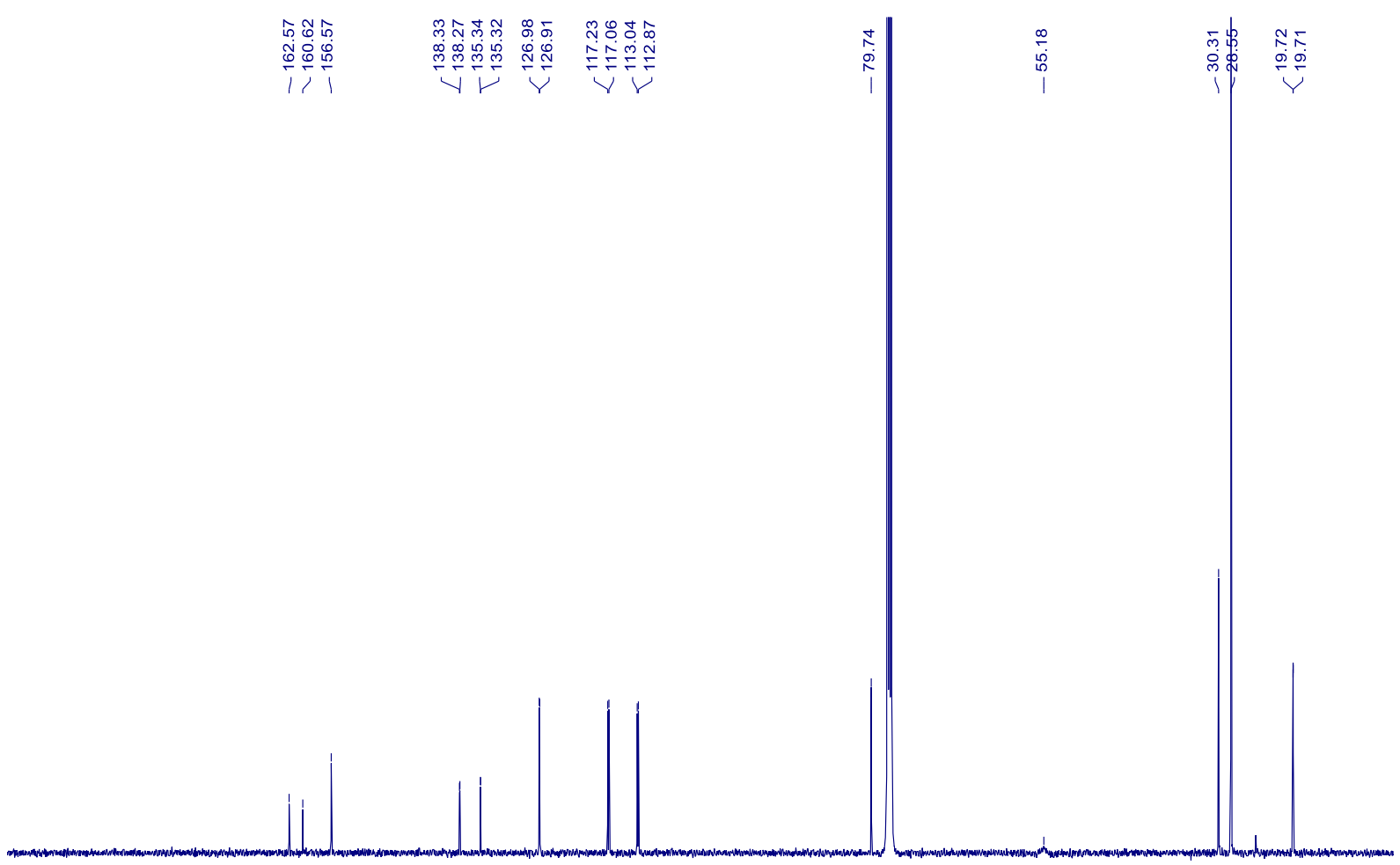

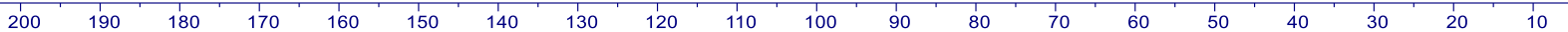


$19-{ }^{19} \mathrm{~F} \mathrm{NMR}\left(376 \mathrm{MHz}, \mathrm{CDCl}_{3}\right)$

$$
\stackrel{\substack{\infty \\ \stackrel{0}{i}}}{i}
$$

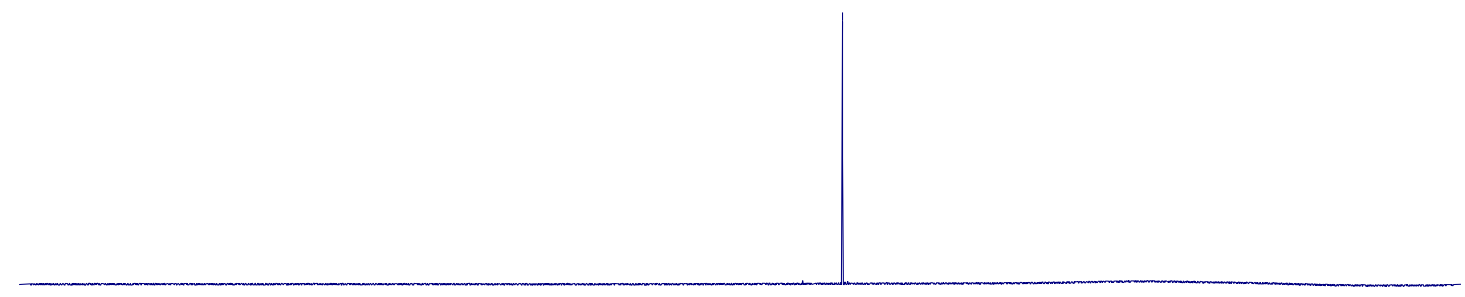

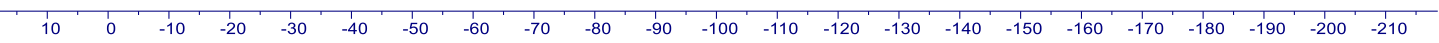


$21-{ }^{1} \mathrm{H}$ NMR $\left(400 \mathrm{MHz}, \mathrm{CDCl}_{3}\right)$
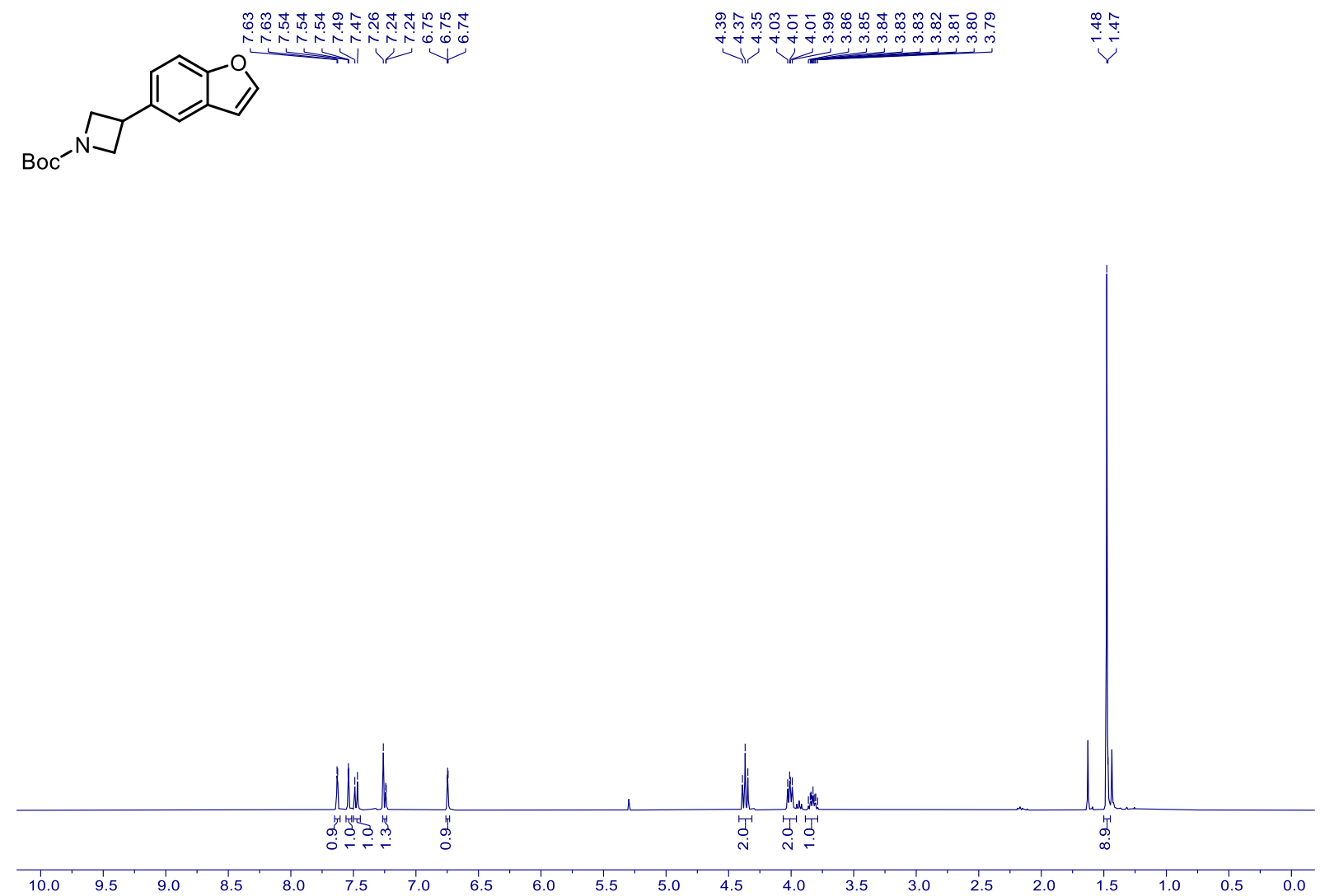

$21-{ }^{13} \mathrm{C}$ NMR (101 MHz, $\left.\mathrm{CDCl}_{3}\right)$

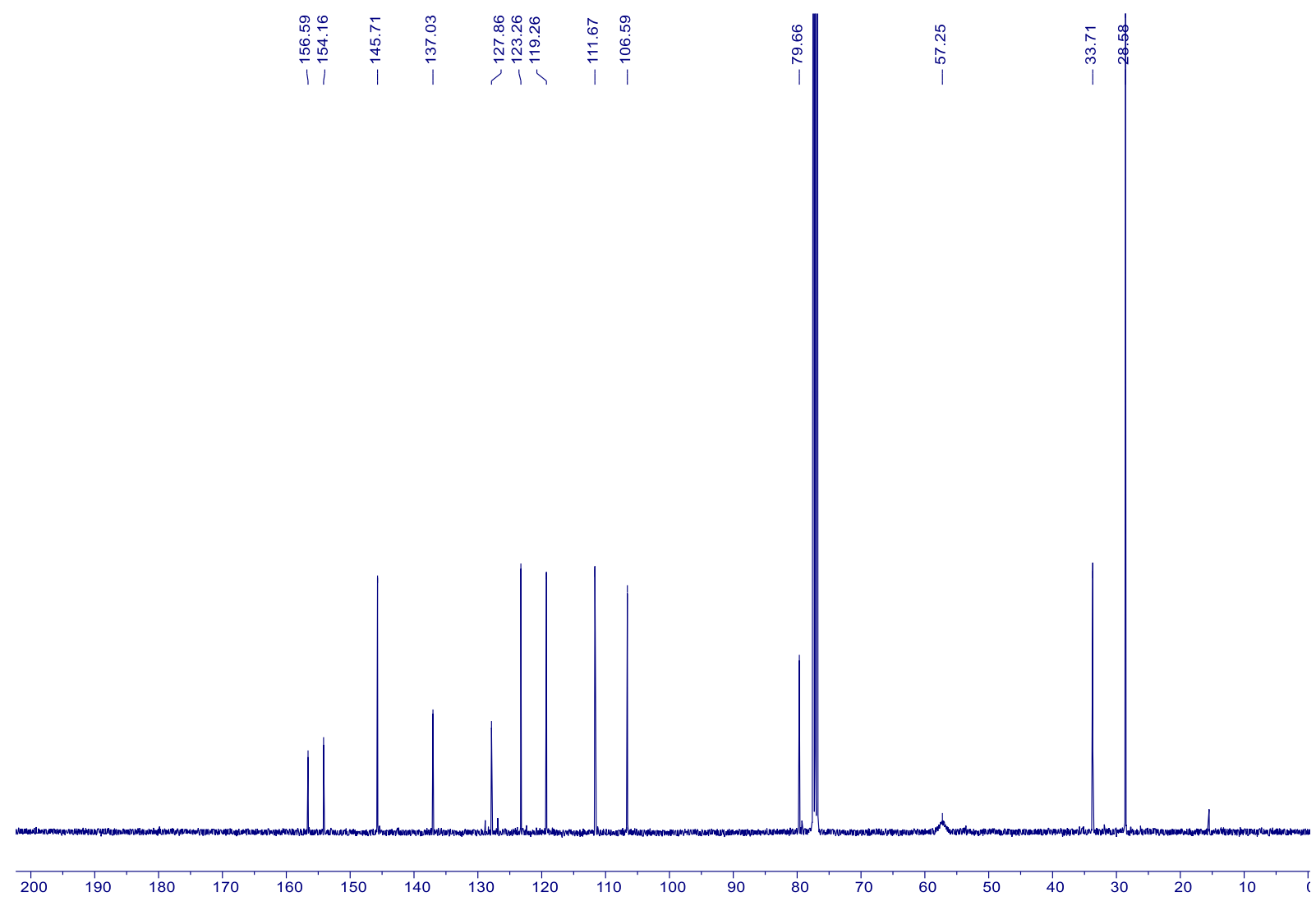


23 - ${ }^{1} \mathrm{H}$ NMR $\left(500 \mathrm{MHz}, \mathrm{CDCl}_{3}\right)$

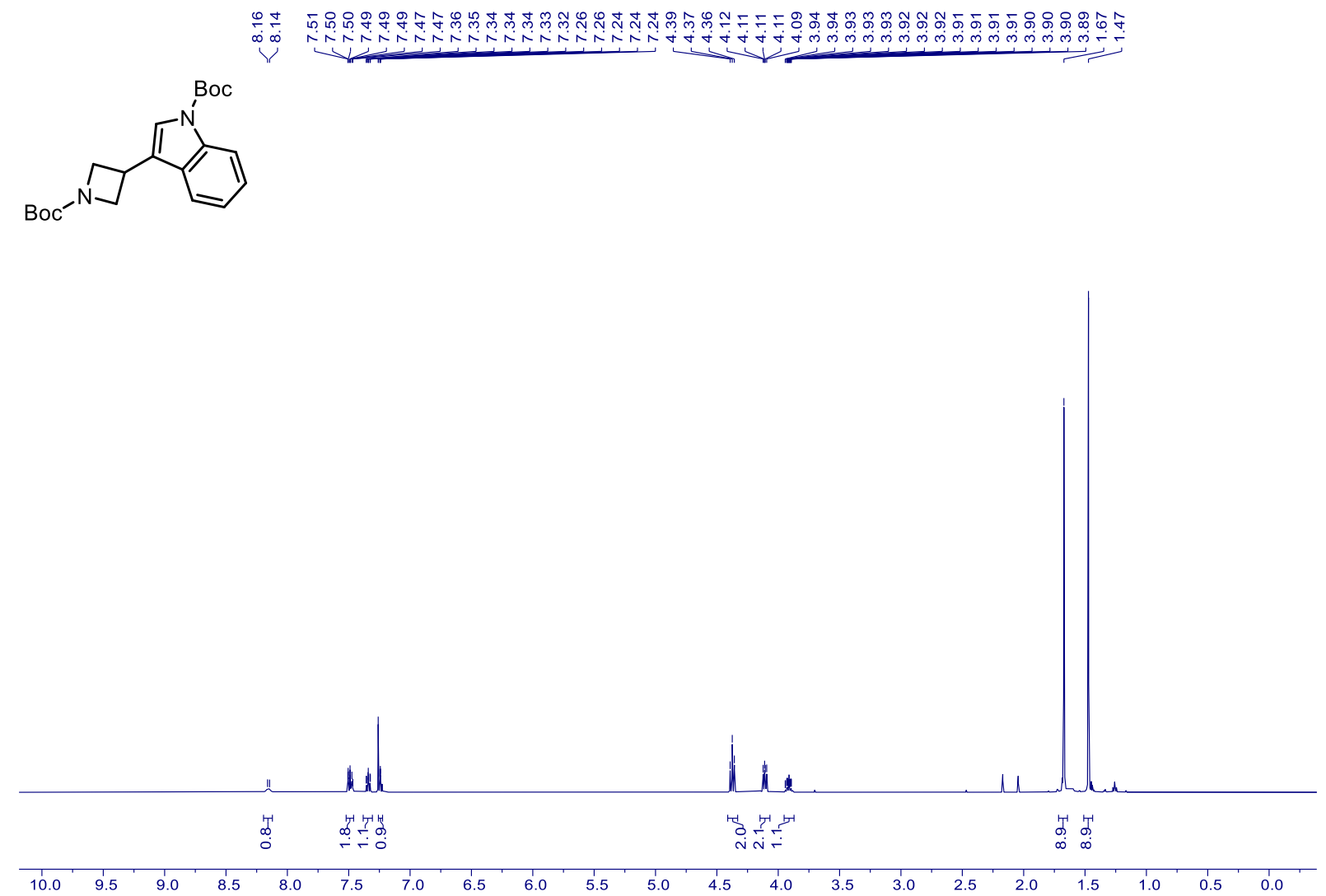

$23-{ }^{13} \mathrm{C}$ NMR (126 MHz, $\left.\mathrm{CDCl}_{3}\right)$

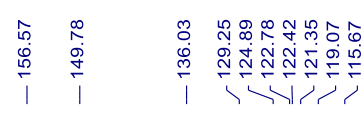

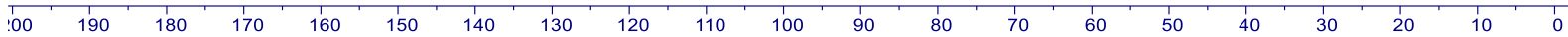


$27-{ }^{1} \mathrm{H}$ NMR (400 MHz, $\mathrm{CDCl}_{3}$ )<smiles>Nn1nccc1C(=O)NC(=O)c1ccccc1</smiles>

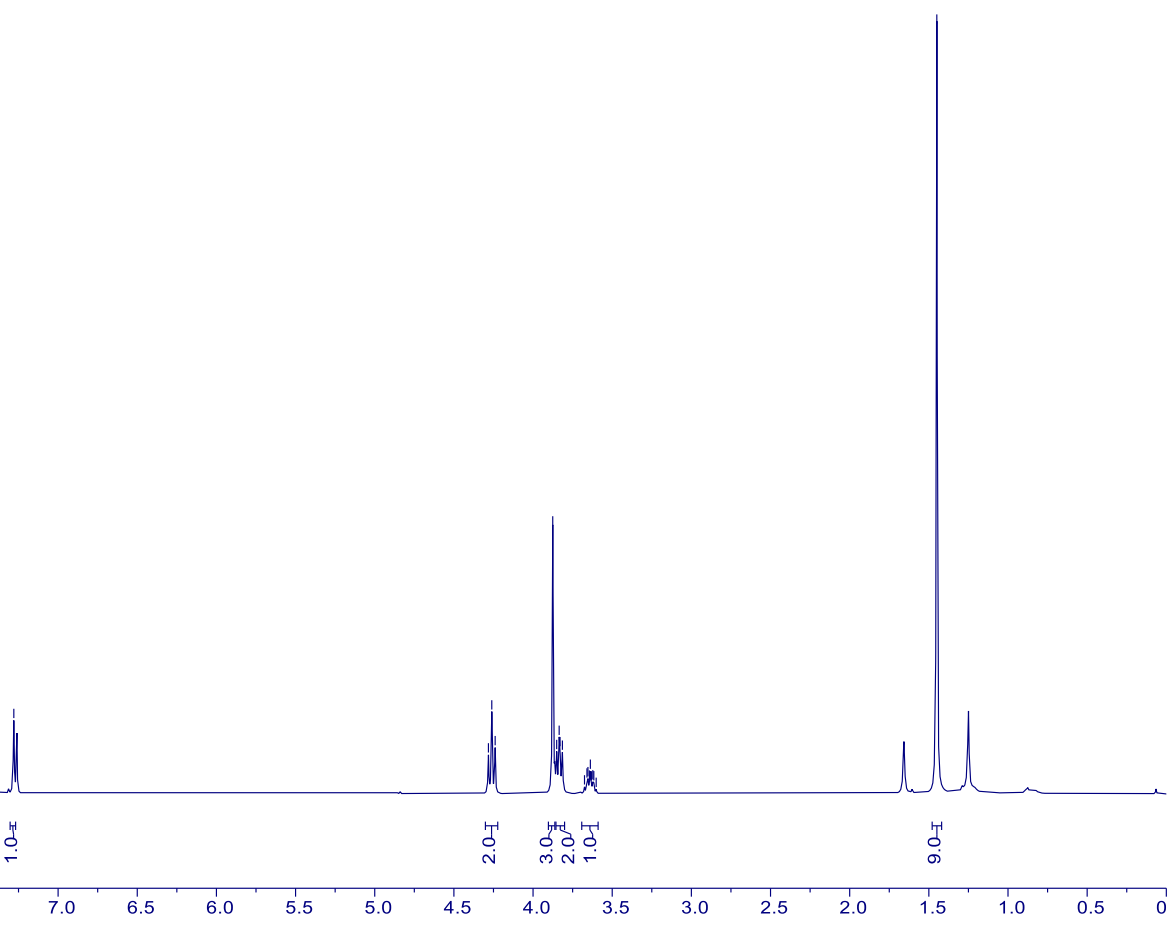

$27-{ }^{13} \mathrm{C} \mathrm{NMR}\left(101 \mathrm{MHz}, \mathrm{CDCl}_{3}\right)$

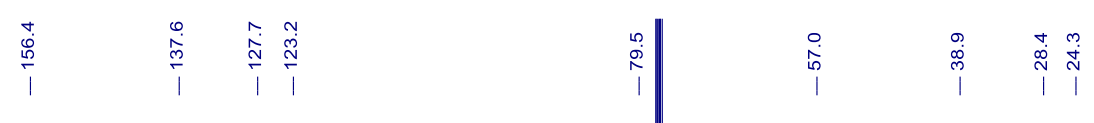

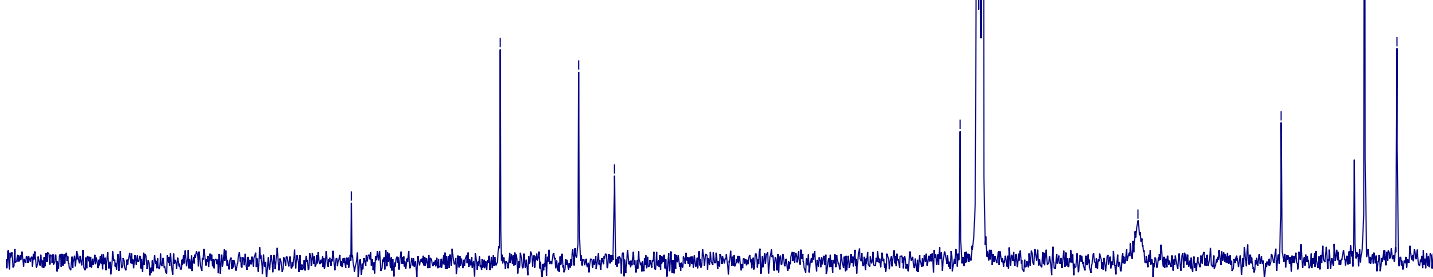


$28-{ }^{1} \mathrm{H}$ NMR $\left(500 \mathrm{MHz}, \mathrm{CDCl}_{3}\right)$
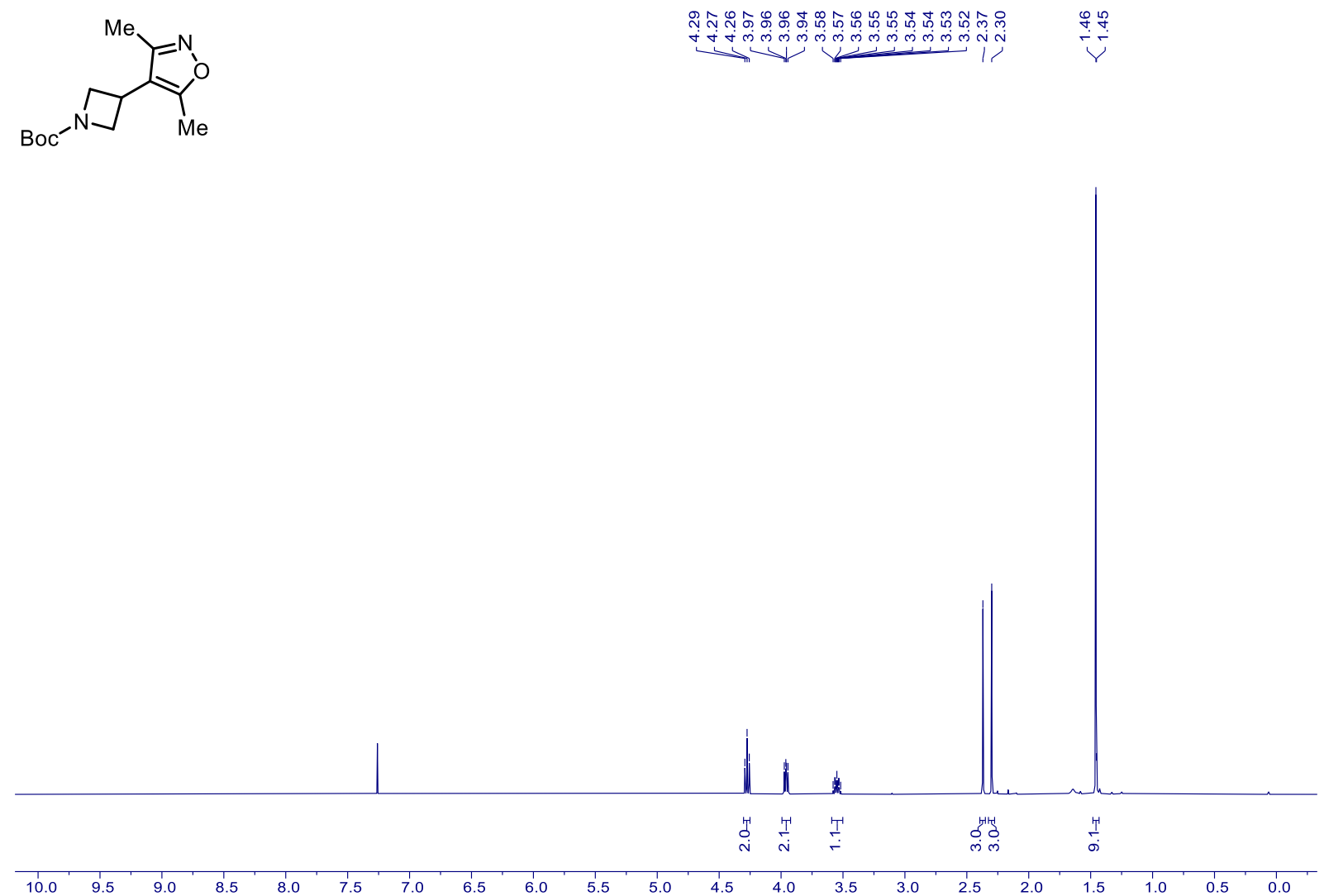

$28-{ }^{13} \mathrm{C}$ NMR $\left(126 \mathrm{MHz}, \mathrm{CDCl}_{3}\right)$

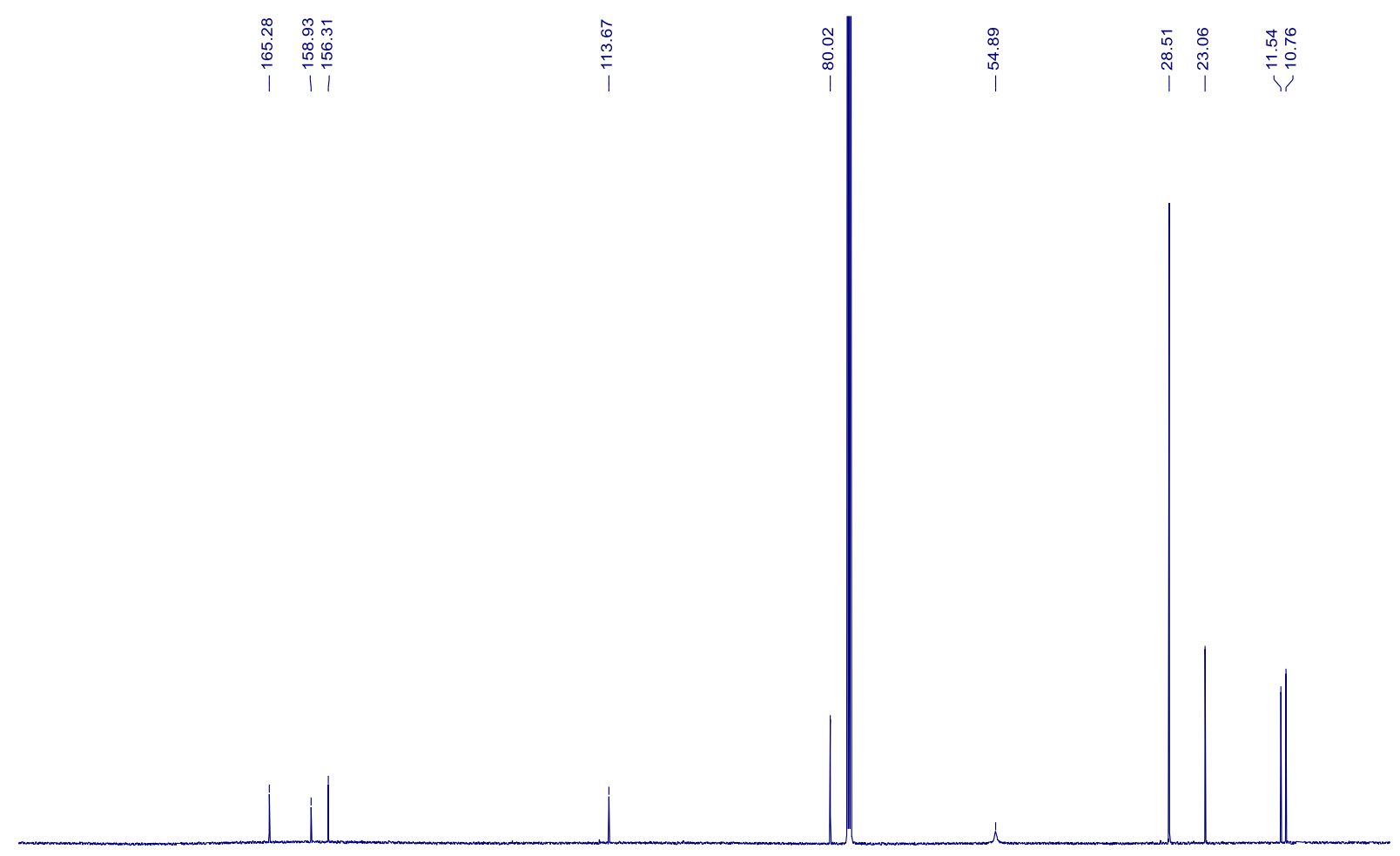

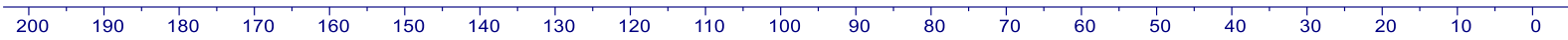


32 - ${ }^{1} \mathrm{H}$ NMR $\left(500 \mathrm{MHz}, \mathrm{CDCl}_{3}\right)$
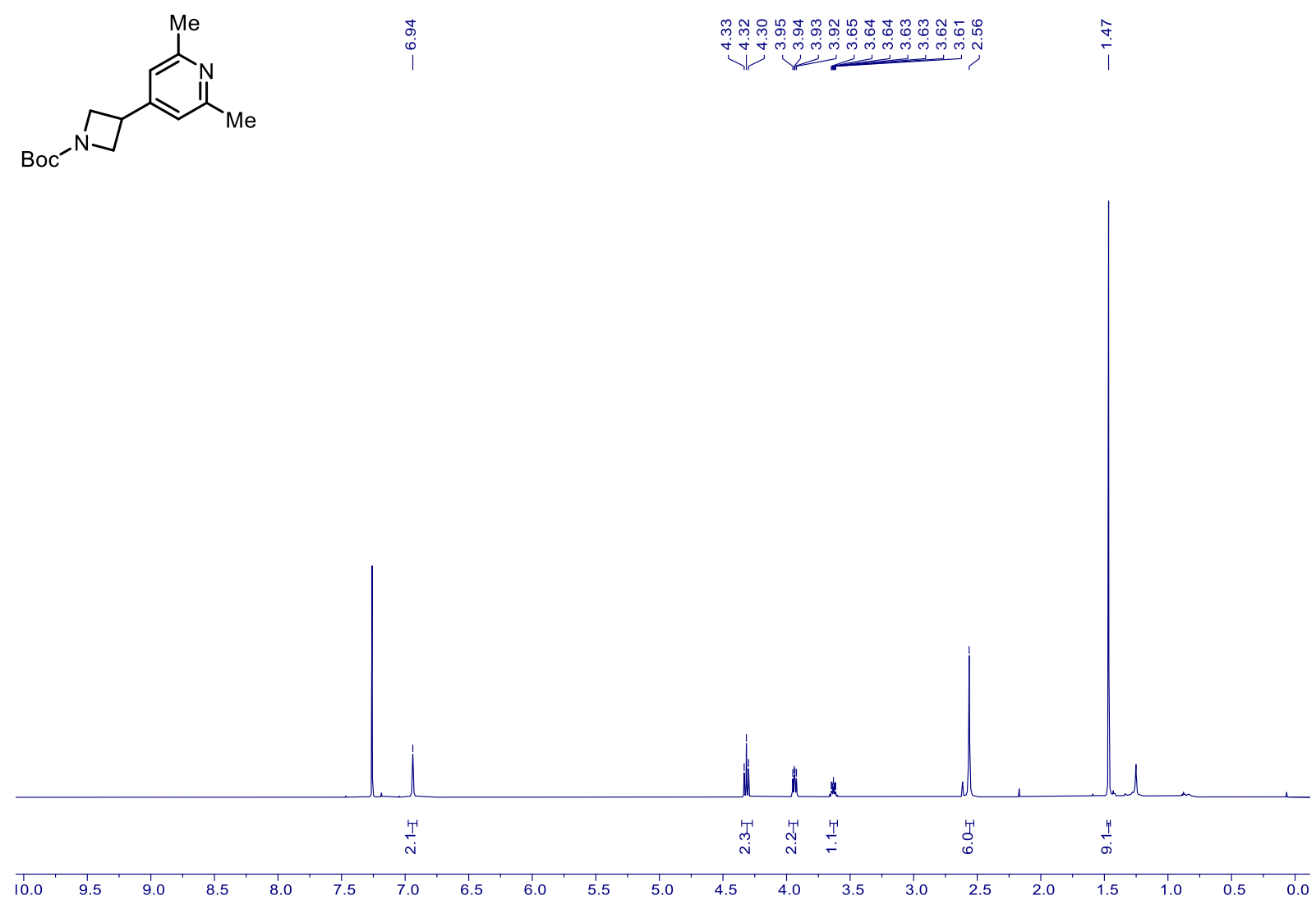

$32-{ }^{13} \mathrm{C}$ NMR $\left(126 \mathrm{MHz}, \mathrm{CDCl}_{3}\right)$

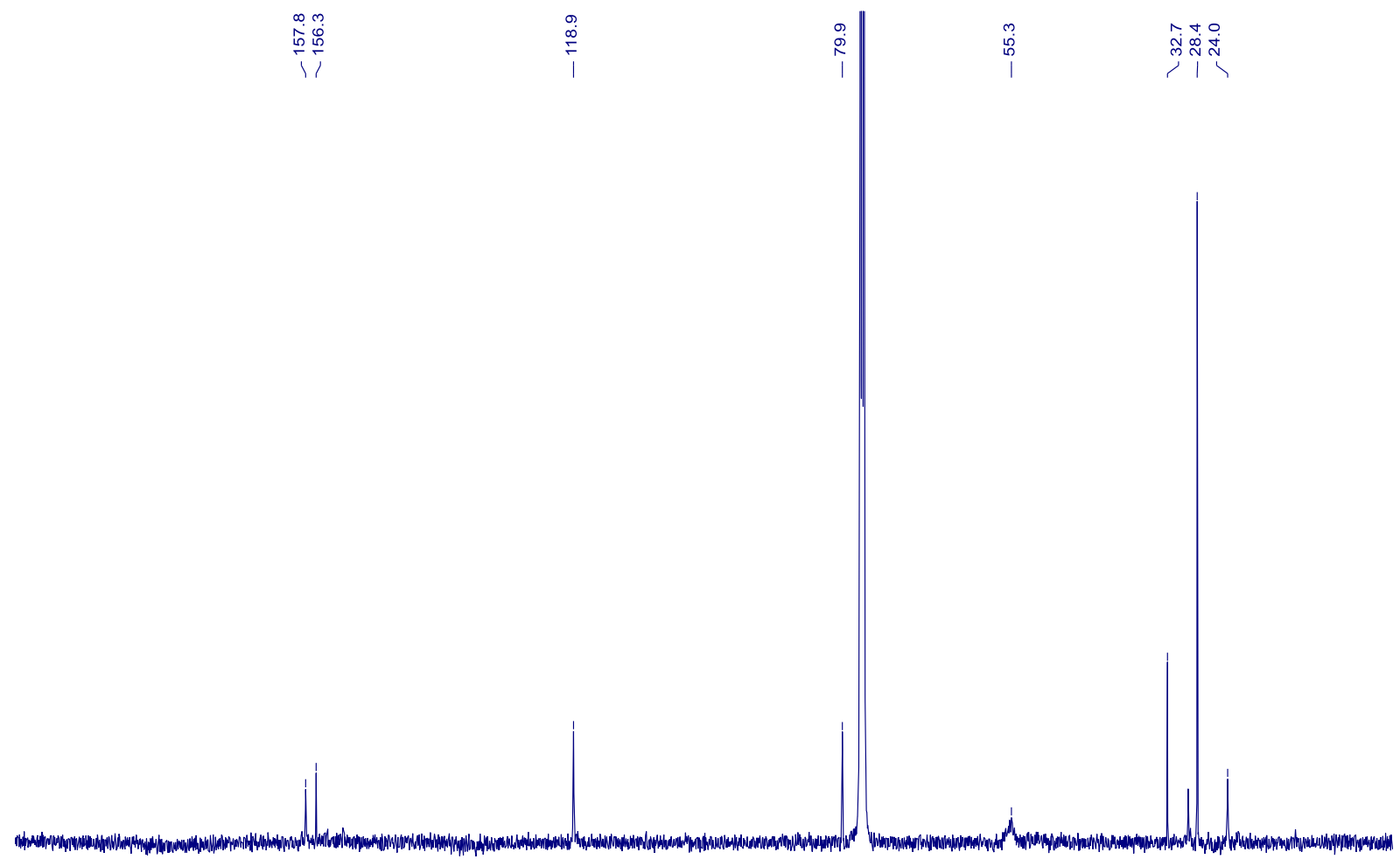

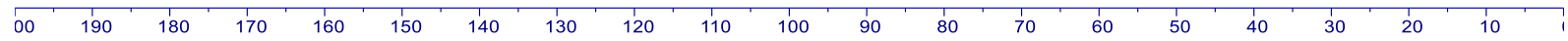


$33-{ }^{1} \mathrm{H}$ NMR $\left(500 \mathrm{MHz}, \mathrm{CDCl}_{3}\right)$

Boc $^{-N} \underbrace{-10}$

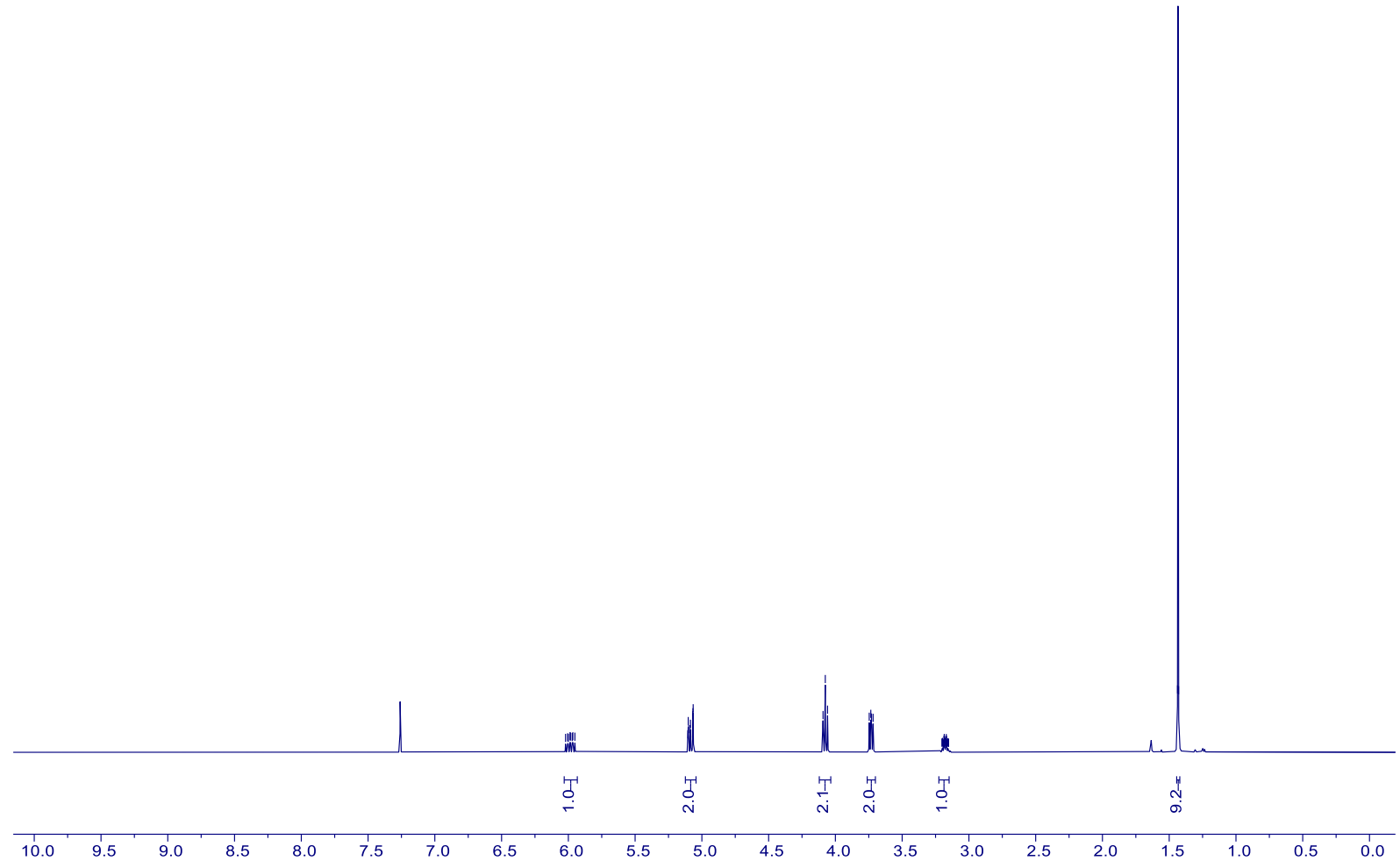

$33-{ }^{13} \mathrm{C}$ NMR $\left(126 \mathrm{MHz}, \mathrm{CDCl}_{3}\right)$

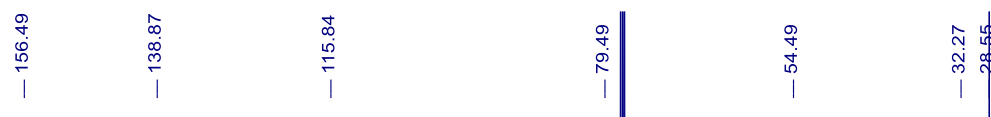

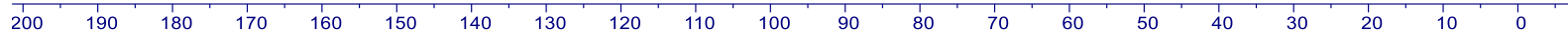


$36-{ }^{1} \mathrm{H}$ NMR $\left(400 \mathrm{MHz}, \mathrm{CDCl}_{3}\right)$

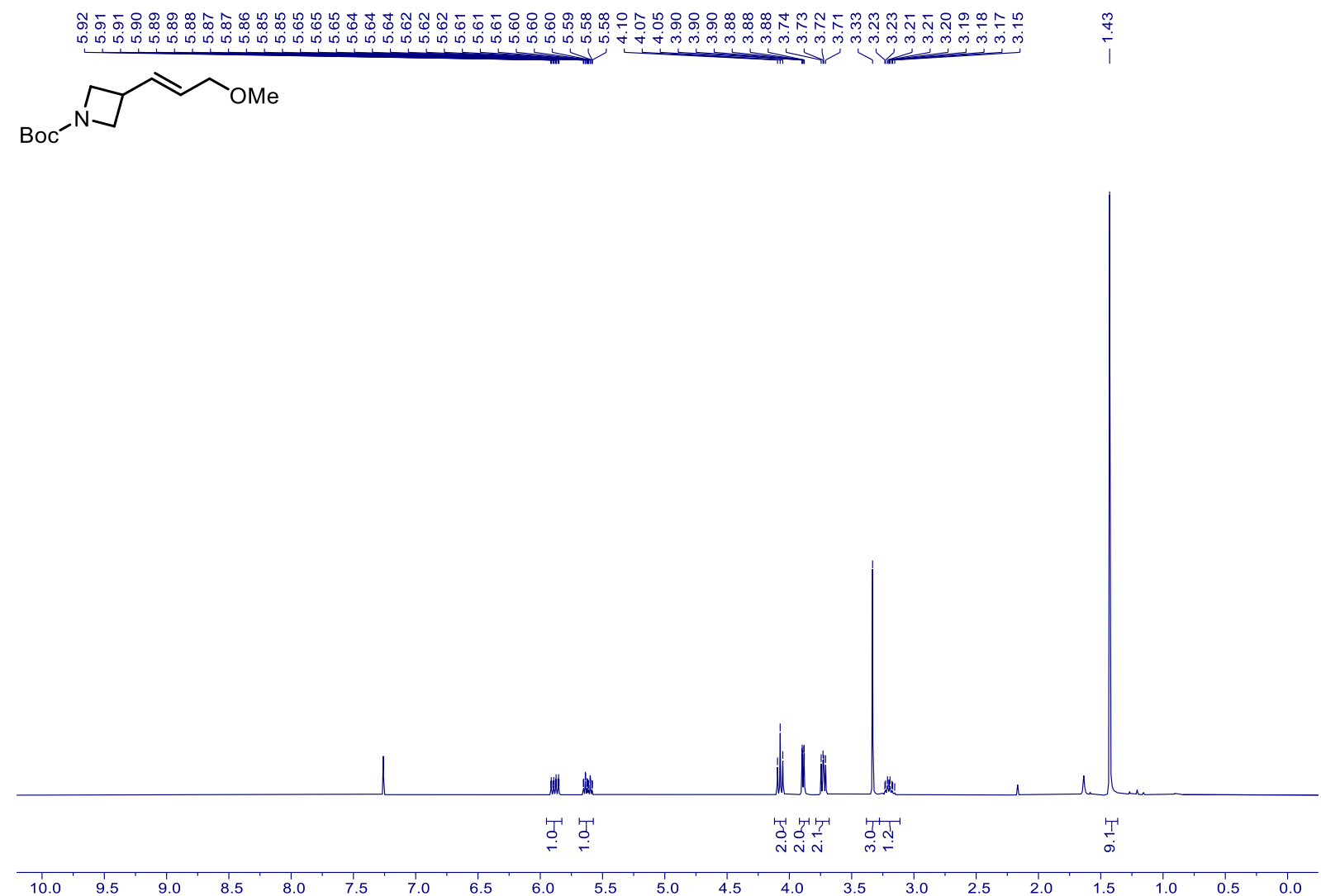

$36-{ }^{13} \mathrm{C}$ NMR $\left(101 \mathrm{MHz}, \mathrm{CDCl}_{3}\right)$

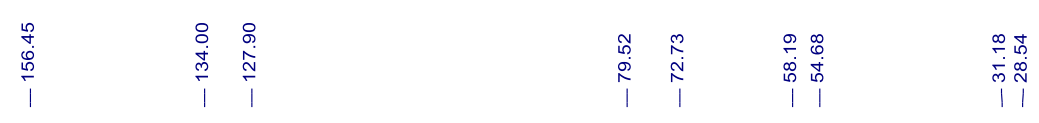

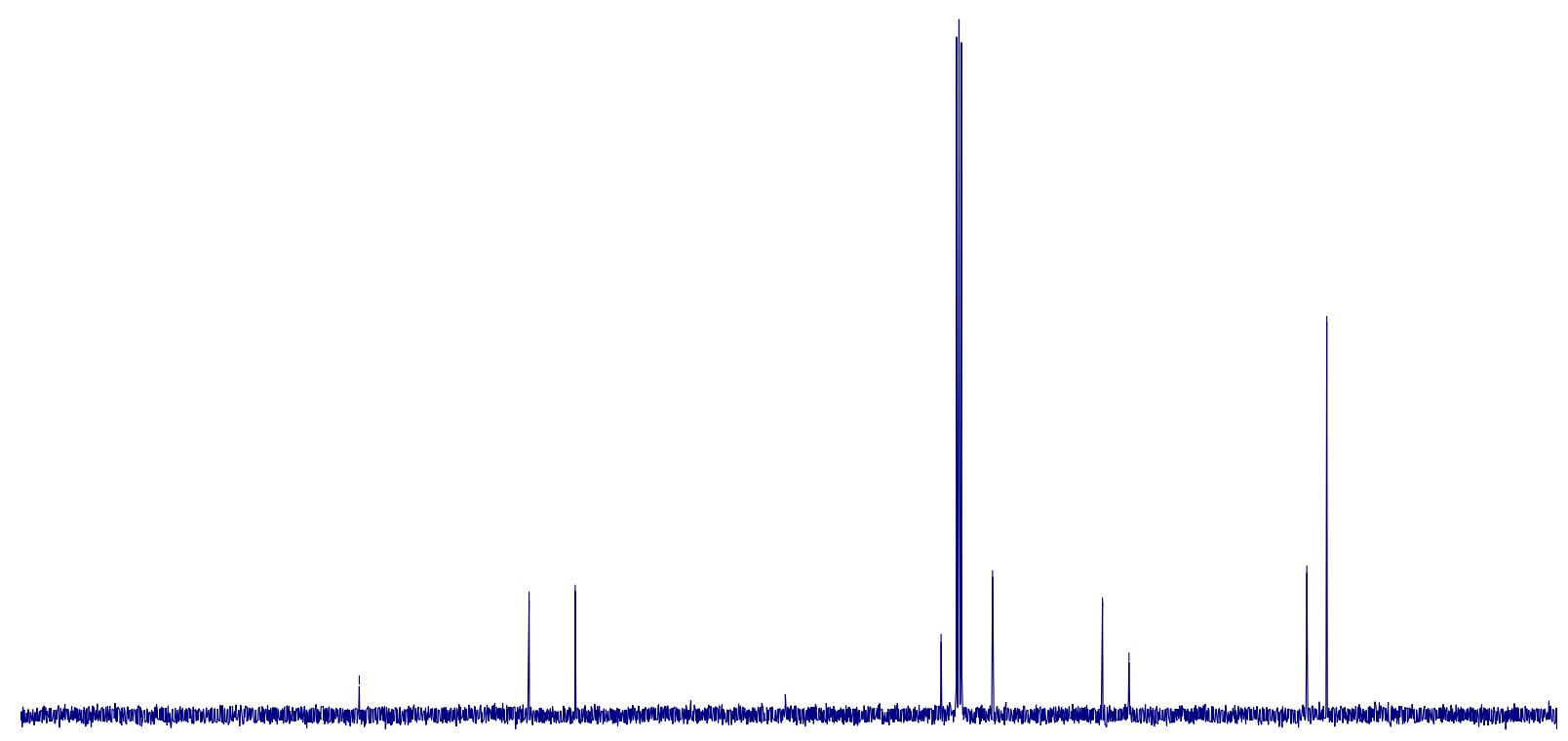

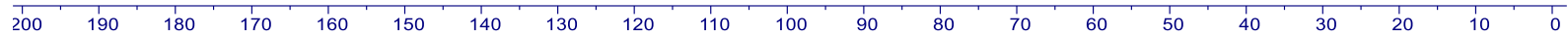


37 - ${ }^{1} \mathrm{H}$ NMR (500 MHz, $\mathrm{CDCl}_{3}$ )

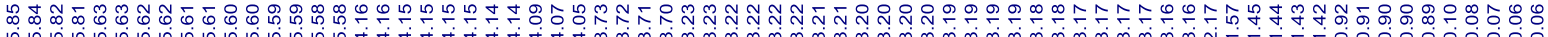
${ }_{\text {Boc }^{-} \mathrm{N}} \mathrm{OTBS}$

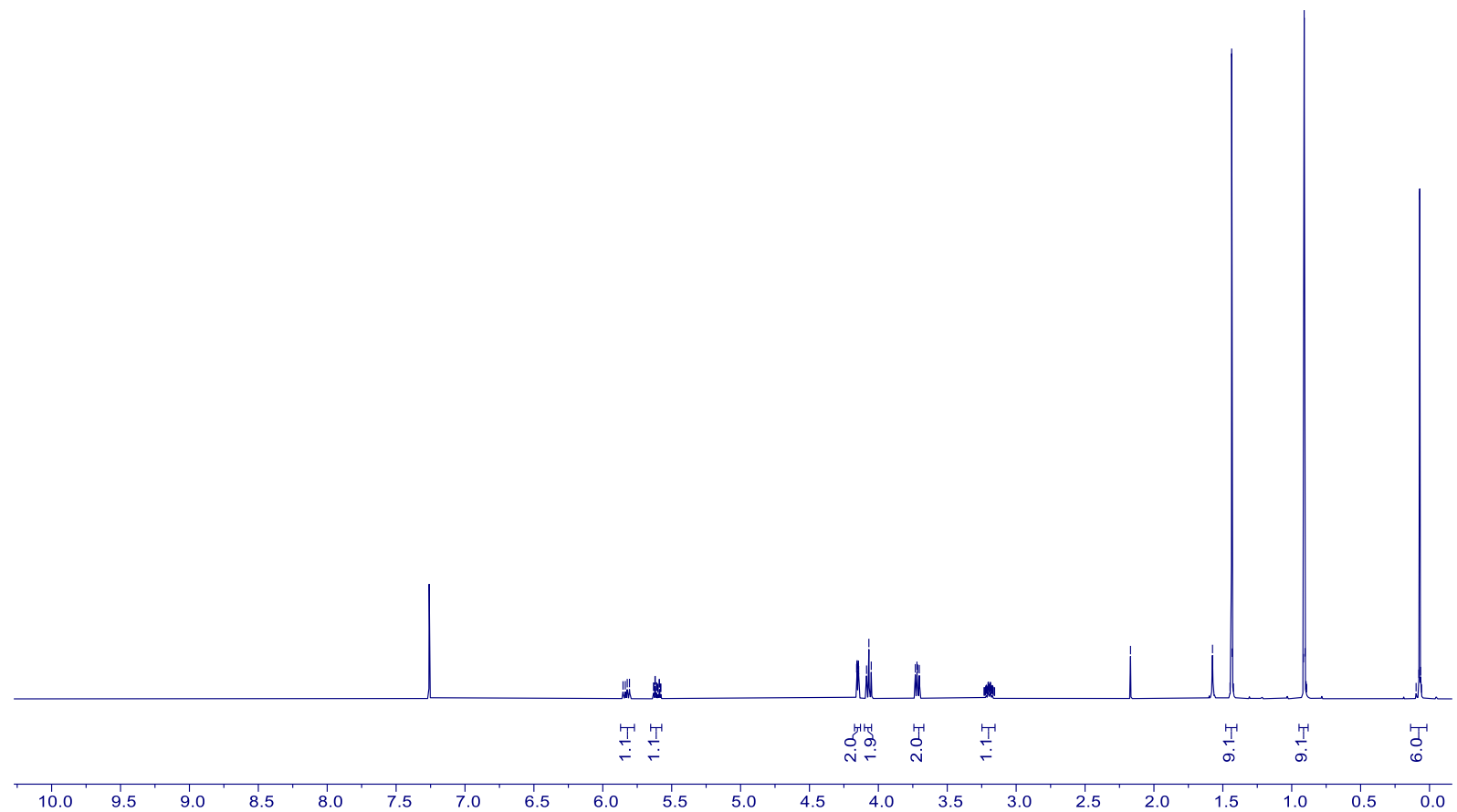

$37-{ }^{13} \mathrm{C}$ NMR $\left(126 \mathrm{MHz}, \mathrm{CDCl}_{3}\right)$

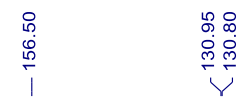

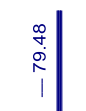

$\begin{array}{ll}9 & 9 \\ 0 & 0 \\ 0 & 0 \\ 0 & 1\end{array}$

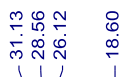

ơ 
38 - ${ }^{1} \mathrm{H}$ NMR $\left(500 \mathrm{MHz}, \mathrm{CDCl}_{3}\right)$<smiles>[R16]C1CNC1C1=CCOC1</smiles>

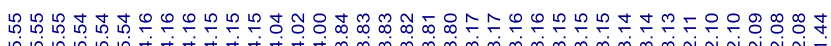

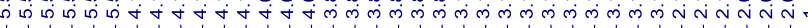

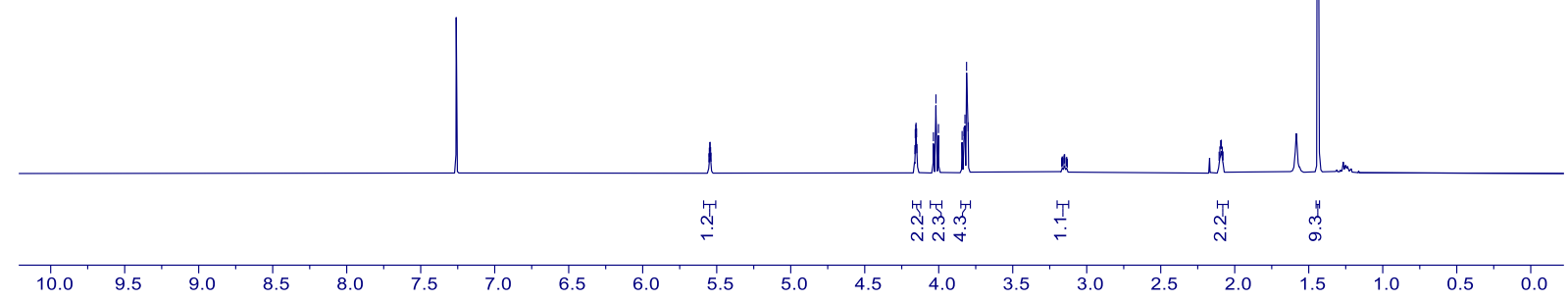

$38-{ }^{13} \mathrm{C}$ NMR $\left(126 \mathrm{MHz}, \mathrm{CDCl}_{3}\right)$

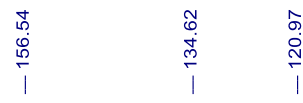

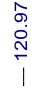

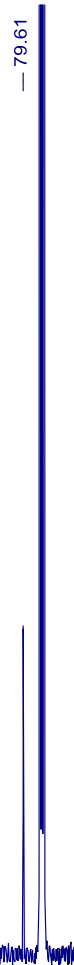

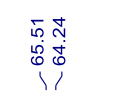

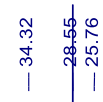


39 - ${ }^{1} \mathrm{H}$ NMR (400 MHz, $\left.\mathrm{CDCl}_{3}\right)$<smiles>CC(C)=CC1CN(C(=O)OC(C)(C)C)C1</smiles>

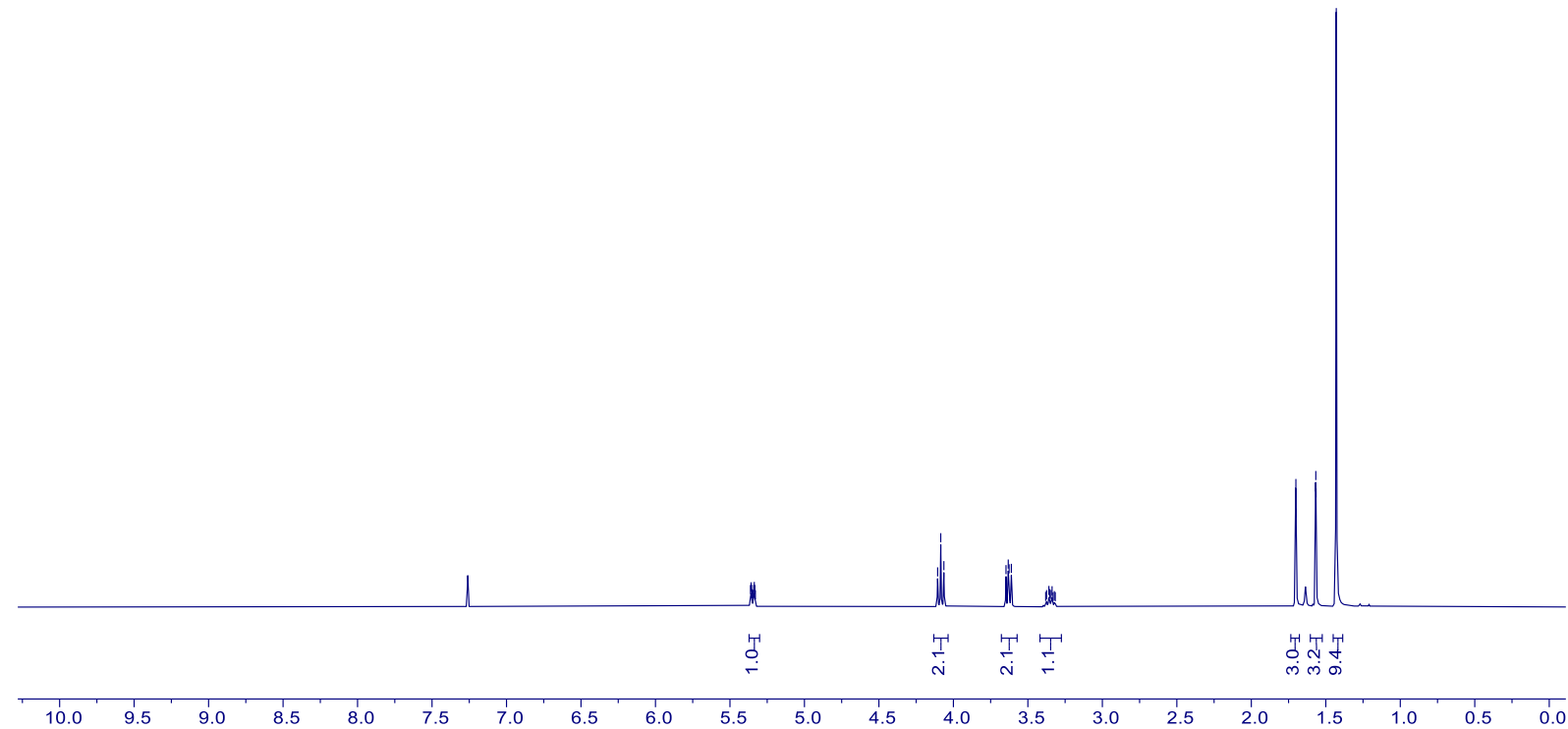

$39-{ }^{13} \mathrm{C}$ NMR $\left(101 \mathrm{MHz}, \mathrm{CDCl}_{3}\right)$

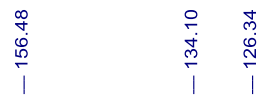


42 - ${ }^{1} \mathrm{H}$ NMR (500 MHz, $\mathrm{CDCl}_{3}$ )

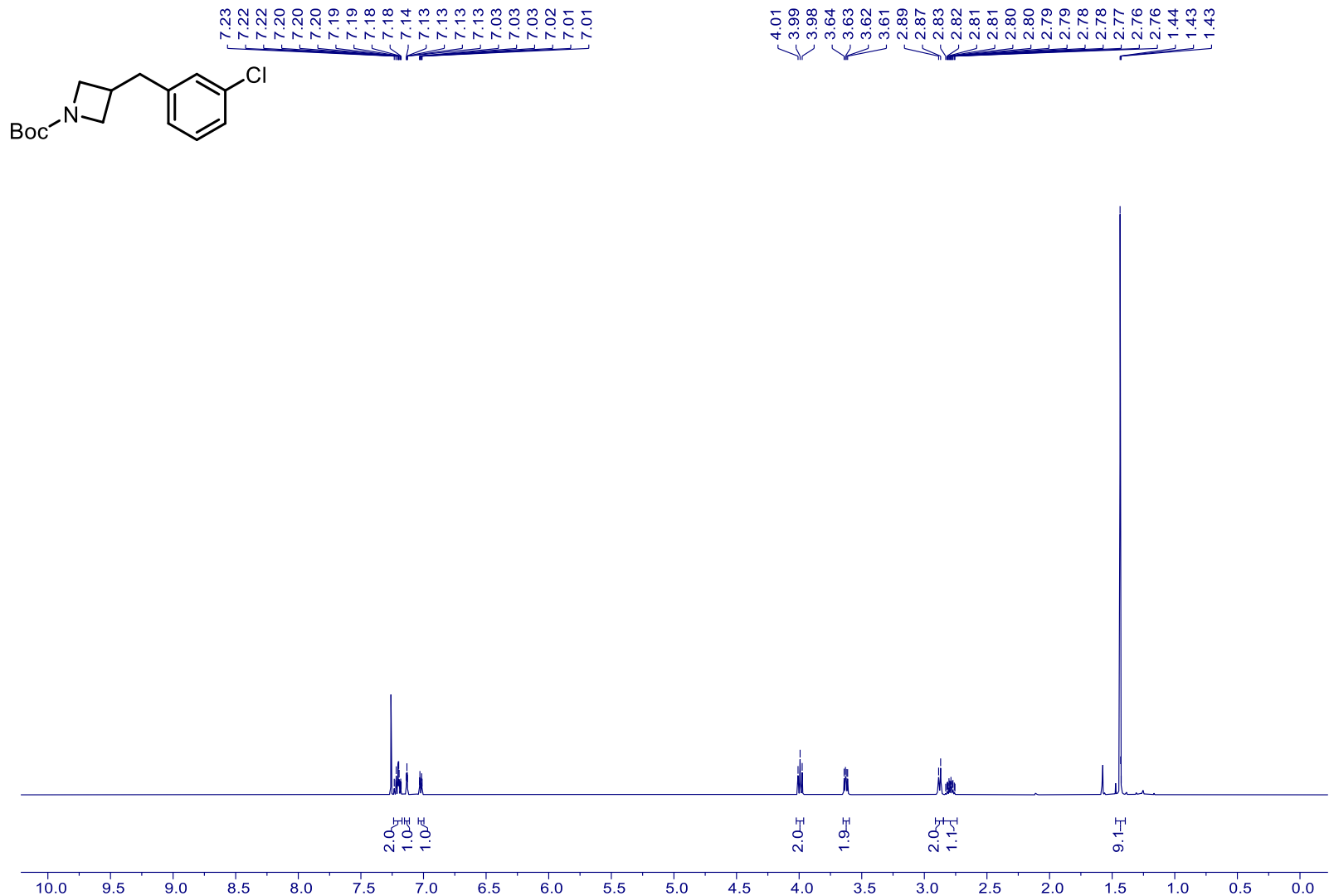

$42-{ }^{13} \mathrm{C} \mathrm{NMR}\left(126 \mathrm{MHz}, \mathrm{CDCl}_{3}\right)$

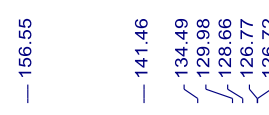

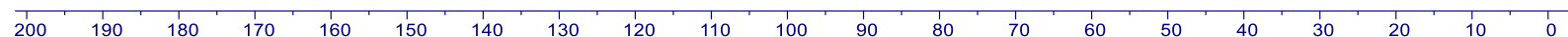


43 - ${ }^{1} \mathrm{H}$ NMR (500 MHz, $\mathrm{CDCl}_{3}$ )

$\mathrm{Boc}^{-\mathrm{N}}$

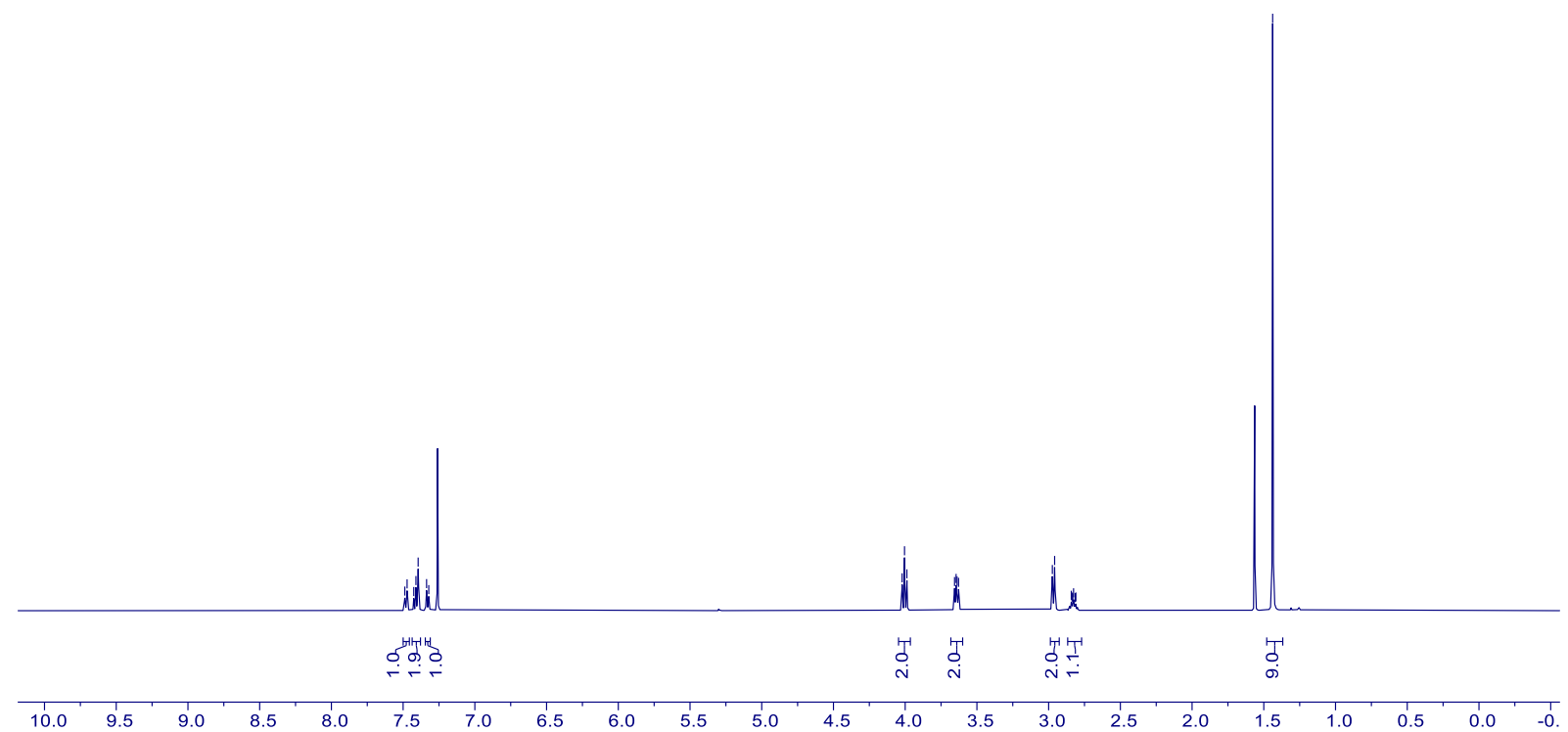

$43-{ }^{13} \mathrm{C}$ NMR (126 MHz, $\left.\mathrm{CDCl}_{3}\right)$

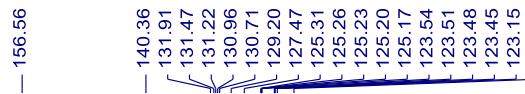

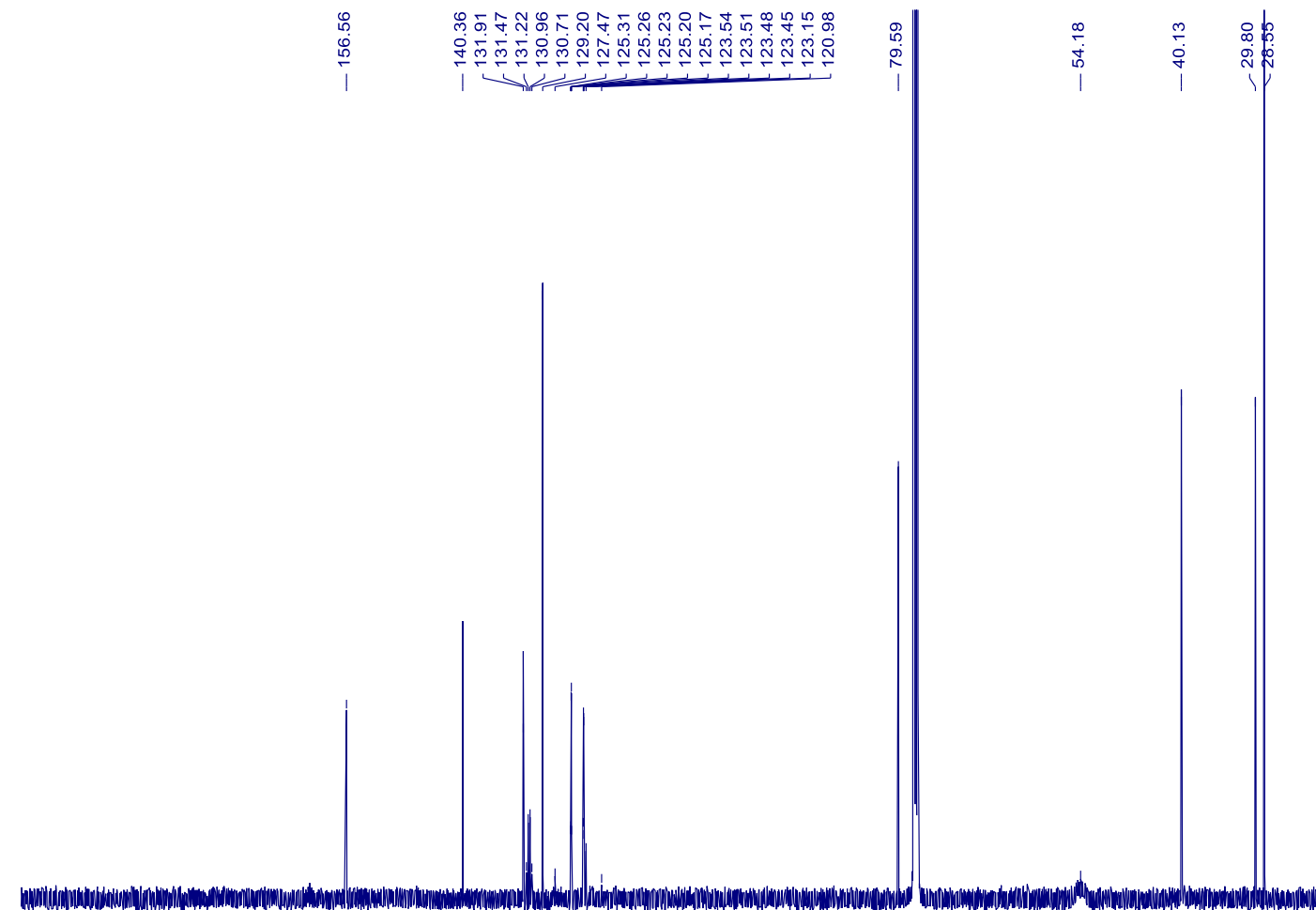

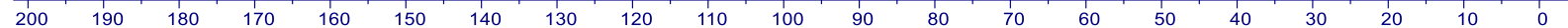


$43-{ }^{19} \mathrm{~F}$ NMR $\left(376 \mathrm{MHz}, \mathrm{CDCl}_{3}\right)$

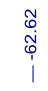

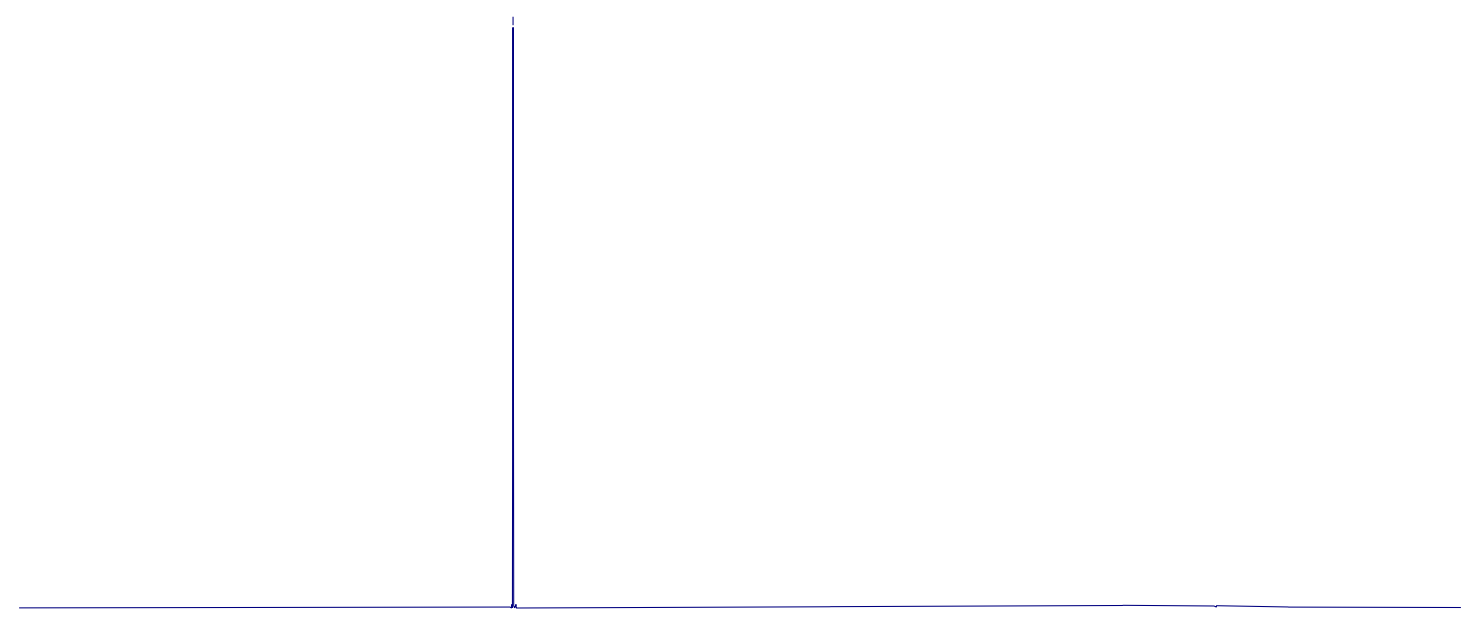

$\begin{array}{lllllllllllllllllllllllll}1 & 10 & 0 & -10 & -20 & -30 & -40 & -50 & -60 & -70 & -80 & -90 & -100 & -110 & -120 & -130 & -140 & -150 & -160 & -170 & -180 & -190 & -100 & -210\end{array}$ 
44 - ${ }^{1} \mathrm{H} \mathrm{NMR}\left(400 \mathrm{MHz}, \mathrm{CDCl}_{3}\right)$

${ }_{\text {Boc }^{-N}} \sqrt{ }$

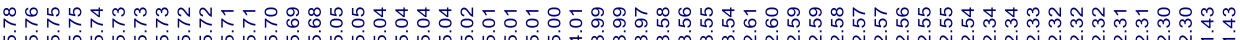

ถ

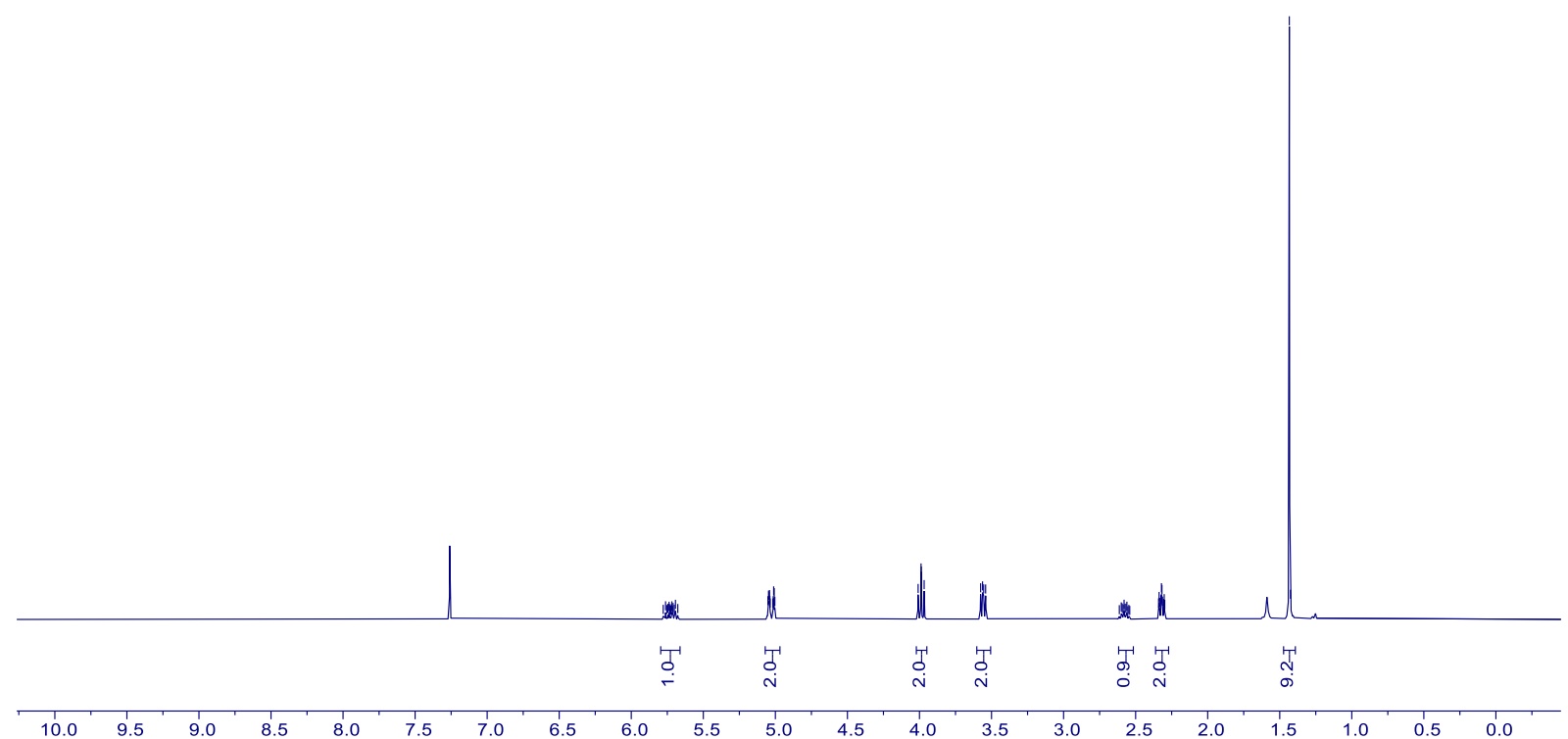

$44-{ }^{13} \mathrm{C}$ NMR $\left(126 \mathrm{MHz}, \mathrm{CDCl}_{3}\right)$

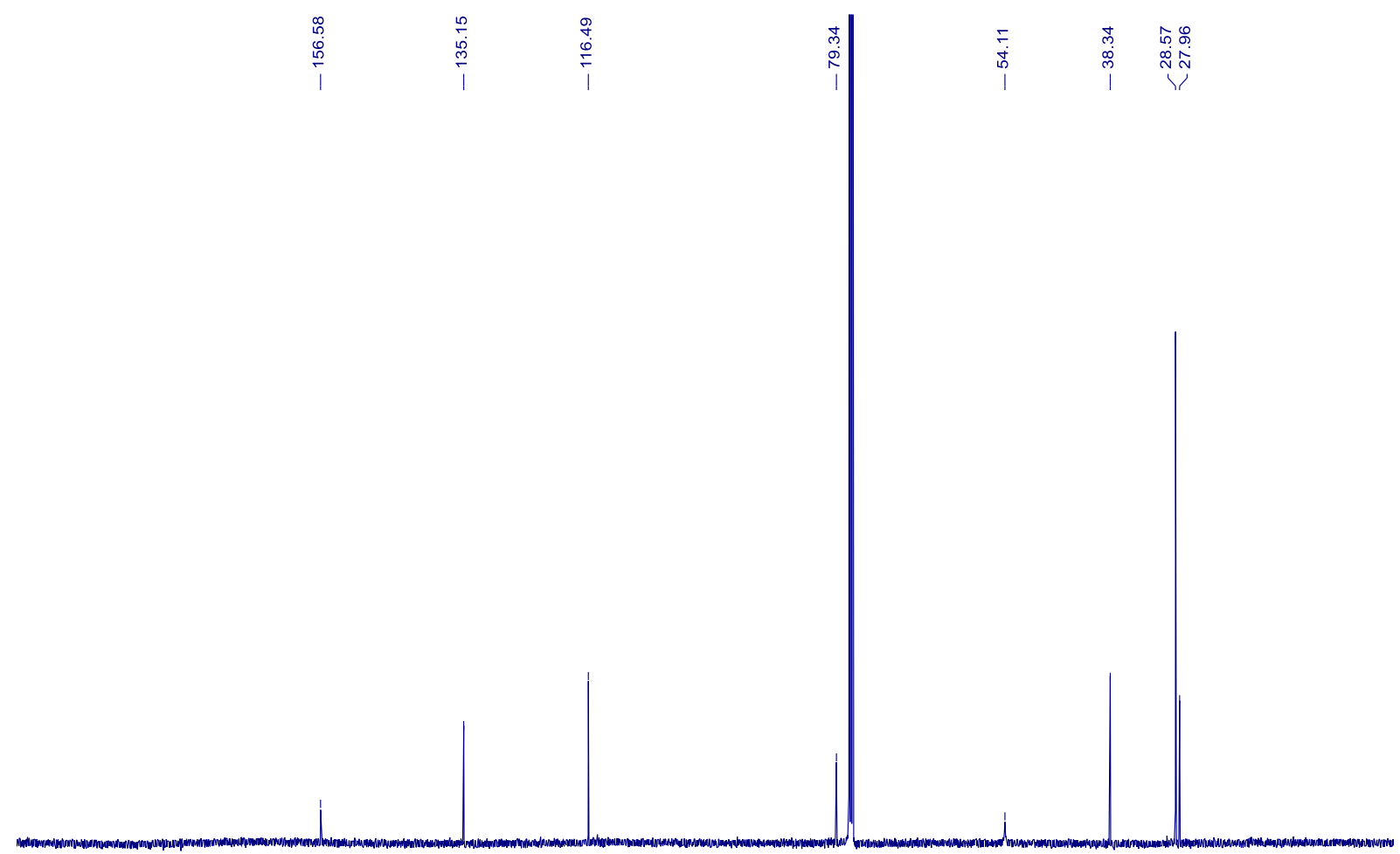

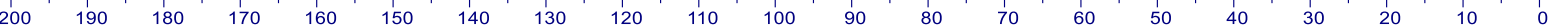


49 - ${ }^{1} \mathrm{H} \mathrm{NMR}\left(500 \mathrm{MHz}, \mathrm{CDCl}_{3}\right)$

ల్య<smiles>c1ccc(C2CCN(c3ncccn3)CC2)cc1</smiles>

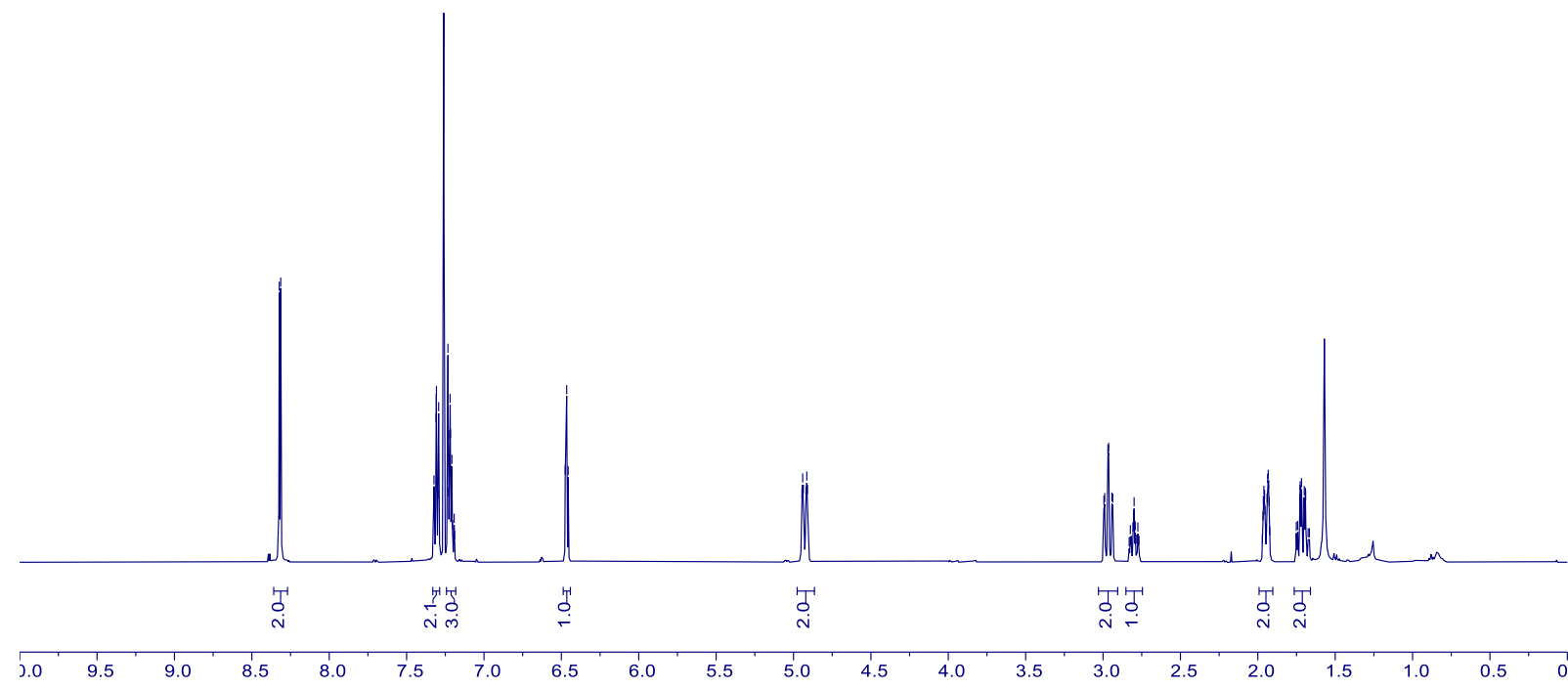

$49-{ }^{13} \mathrm{C} \mathrm{NMR}\left(126 \mathrm{MHz}, \mathrm{CDCl}_{3}\right)$

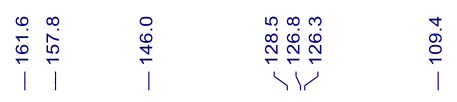


50 - ${ }^{1} \mathrm{H}$ NMR $\left(400 \mathrm{MHz}, \mathrm{CDCl}_{3}\right)$

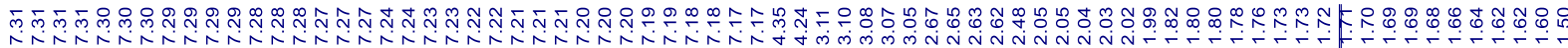<smiles>O=C(c1ccccc1)N1CCC2CCC1C2</smiles>

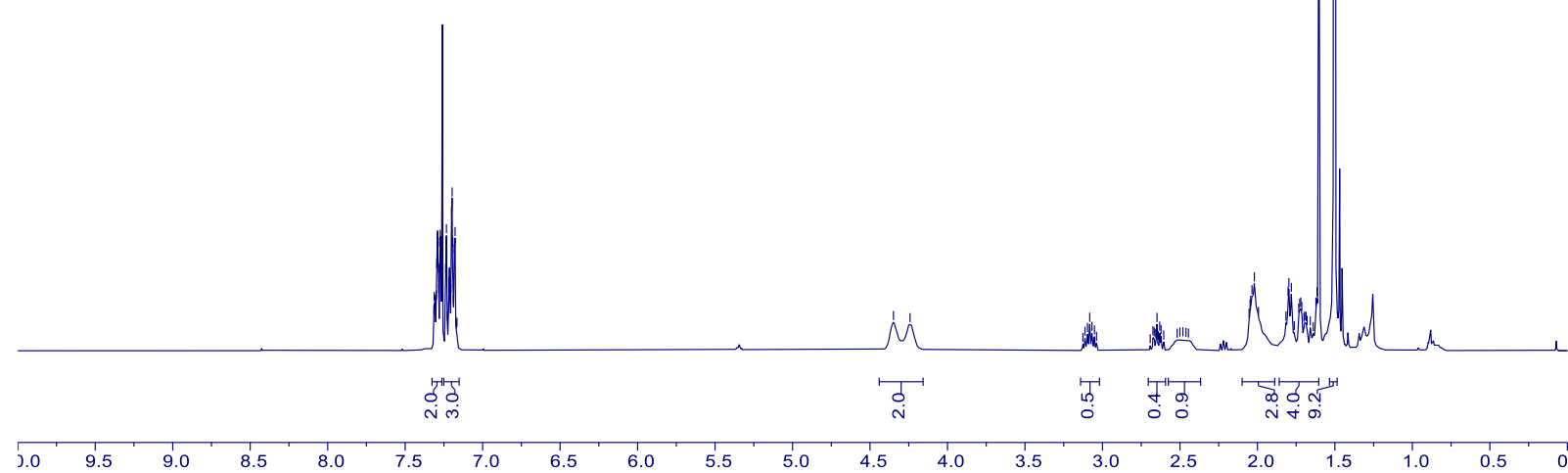

50 - ${ }^{13} \mathrm{C}$ NMR (101 MHz, $\left.\mathrm{CDCl}_{3}\right)$

Vl
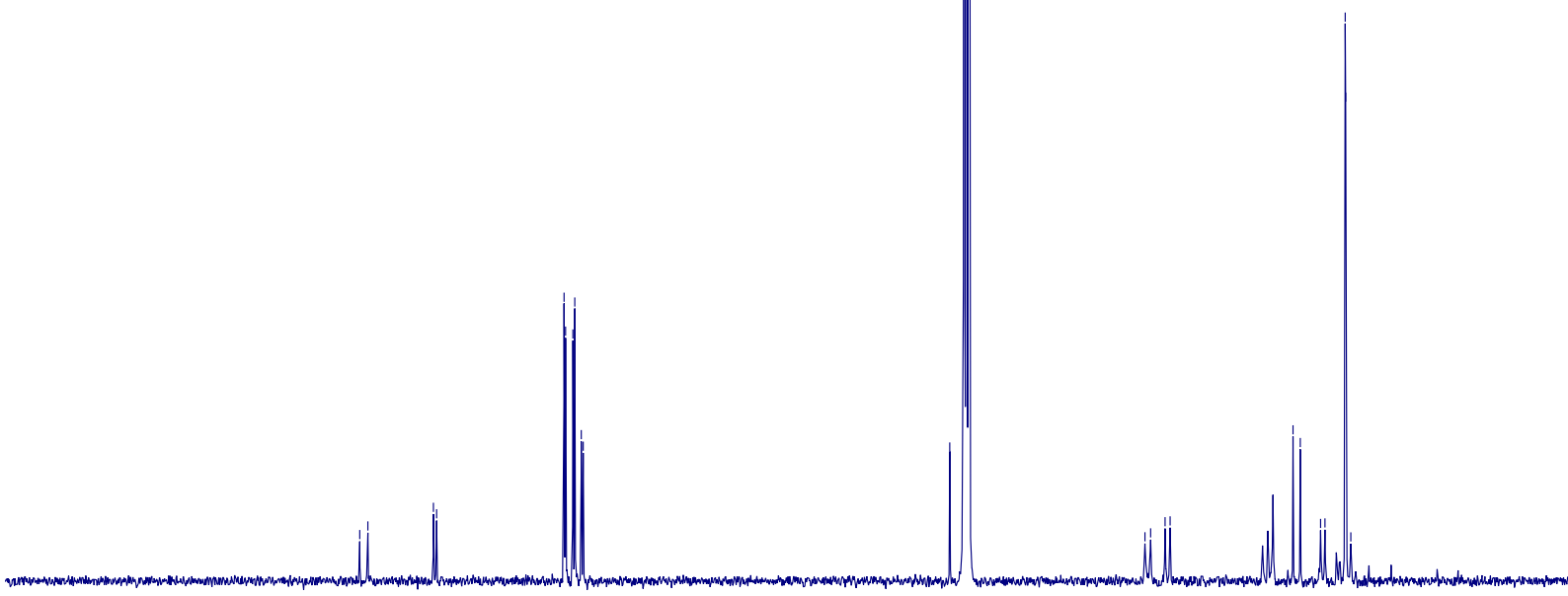

\begin{tabular}{lllllllllllllllllllll}
\hline 00 & 190 & 180 & 170 & 160 & 150 & 140 & 130 & 120 & 110 & 100 & 90 & 80 & 70 & 60 & 50 & 40 & 30 & 20 & 10 & 10
\end{tabular} 
52 - ${ }^{1} \mathrm{H}$ NMR (500 MHz, $\left.\mathrm{CDCl}_{3}\right)$

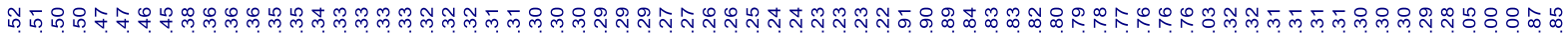

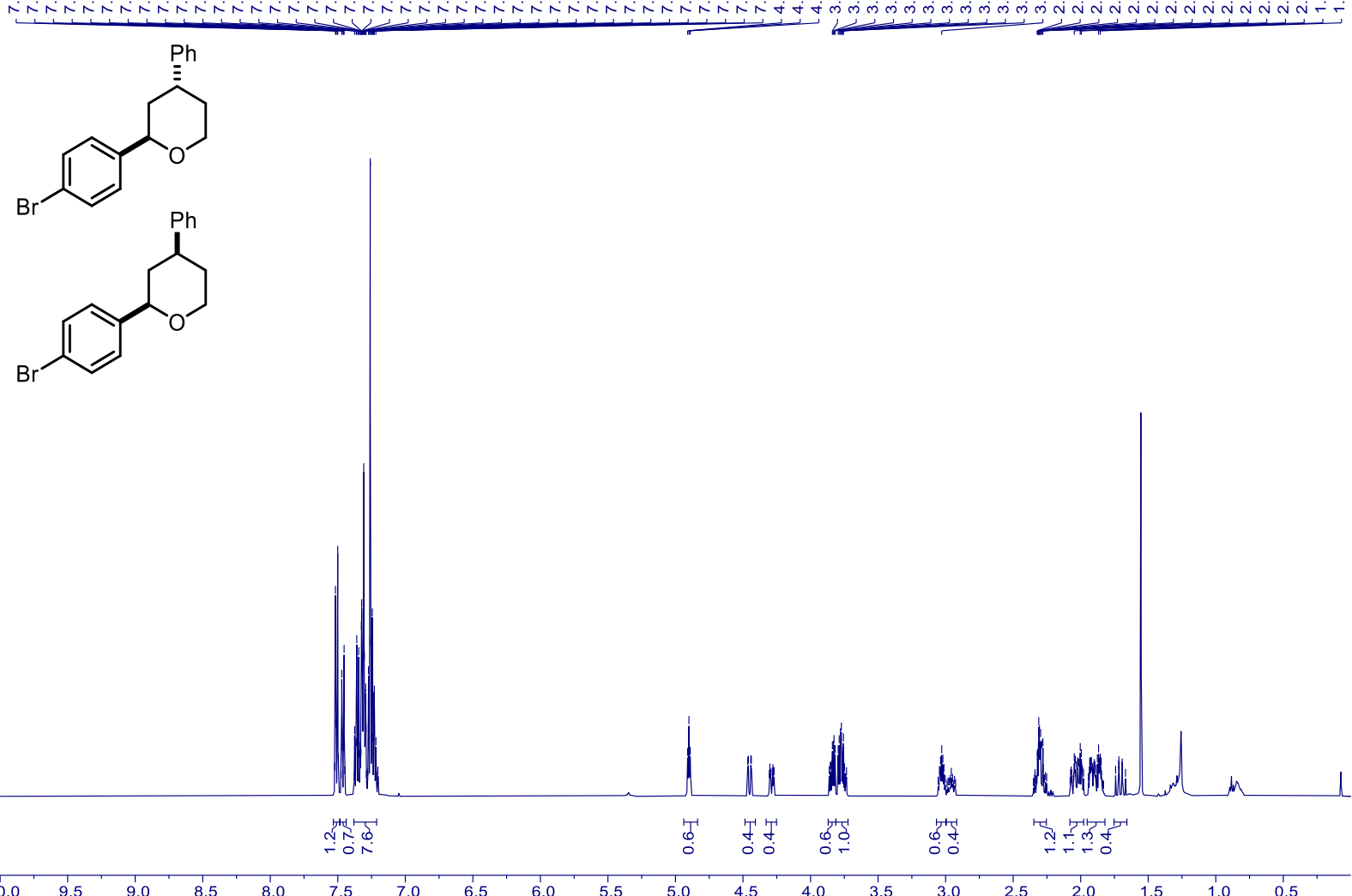

$52-{ }^{13} \mathrm{C}$ NMR $\left(126 \mathrm{MHz}, \mathrm{CDCl}_{3}\right)$

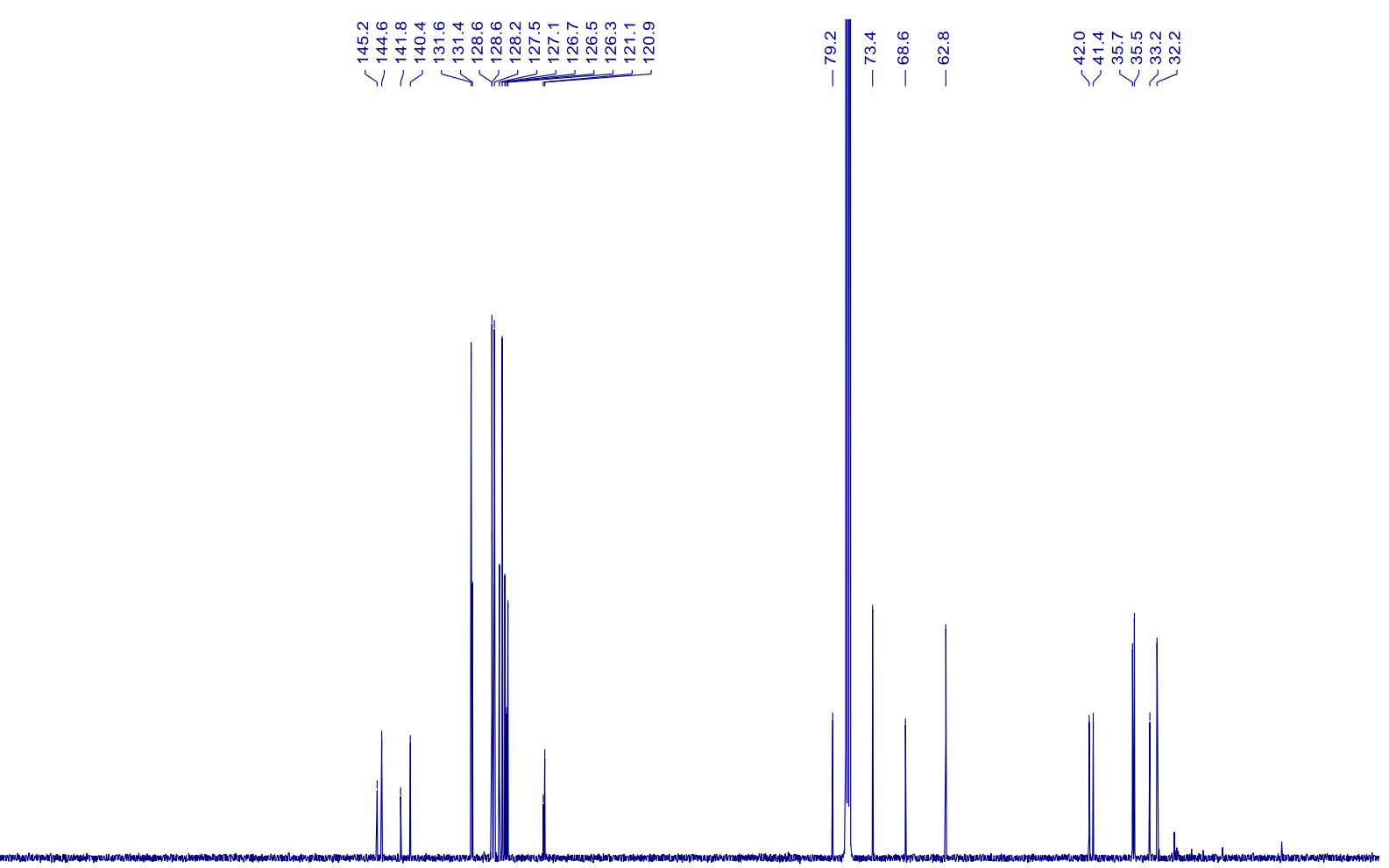

\begin{tabular}{lllllllllllllllllllll}
\hline 00 & 190 & 180 & 170 & 160 & 150 & 140 & 130 & 120 & 110 & 100 & 90 & 80 & 70 & 60 & 50 & 40 & 30 & 20 & 10 & 1
\end{tabular} 
53 - ${ }^{1} \mathrm{H}$ NMR $\left(500 \mathrm{MHz}, \mathrm{CDCl}_{3}\right)$

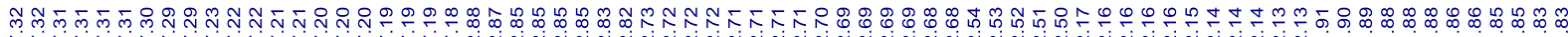

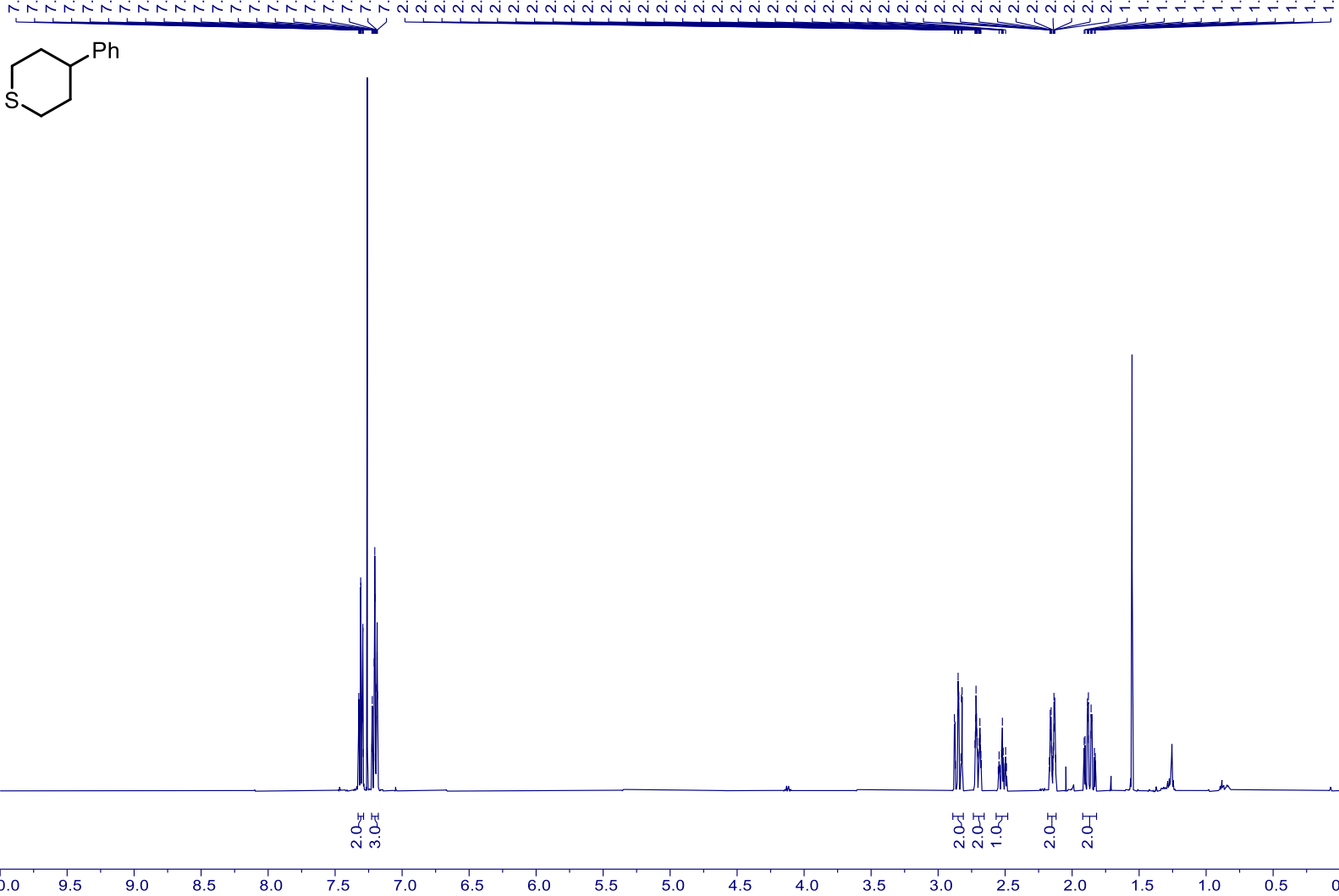

$53-{ }^{13} \mathrm{C}$ NMR $\left(126 \mathrm{MHz}, \mathrm{CDCl}_{3}\right)$

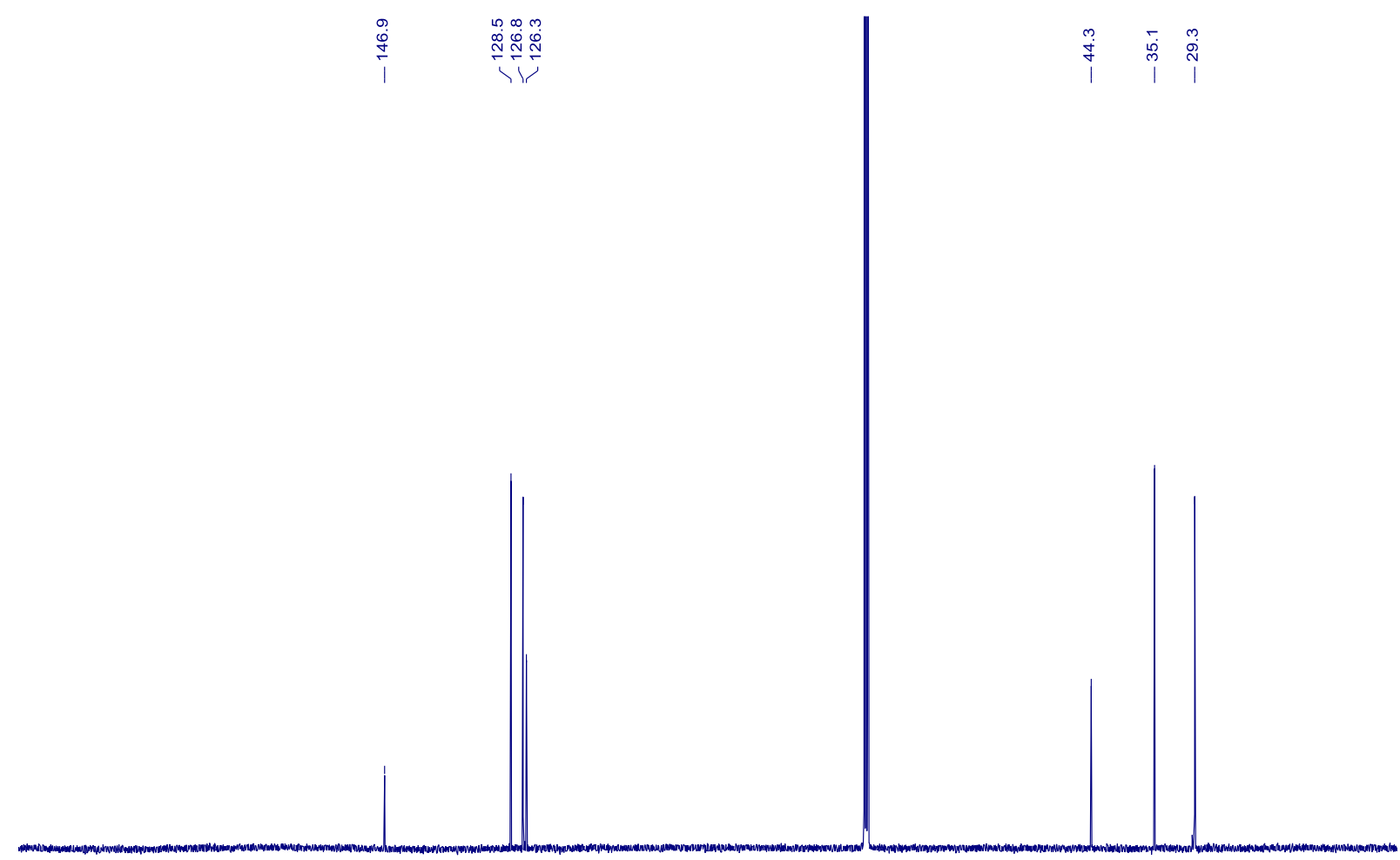

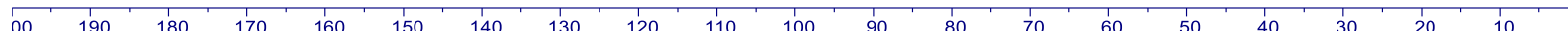


55 - ${ }^{1} \mathrm{H}$ NMR (400 MHz, $\left.\mathrm{CDCl}_{3}\right)$

סำ<smiles>CCOC(=O)C1(C(=O)O)CC(c2ccccc2)C1</smiles>

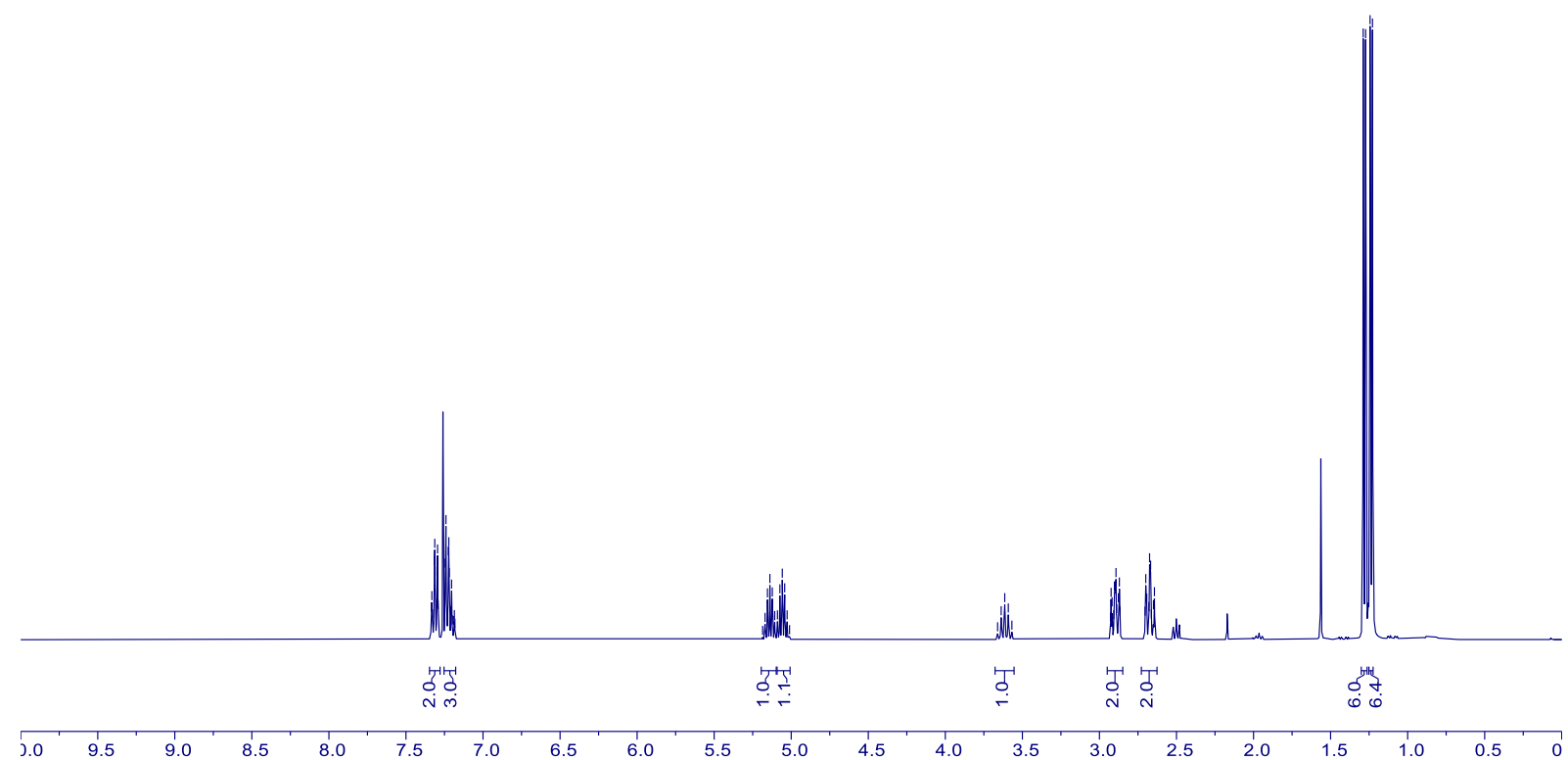

$55-{ }^{13} \mathrm{C}$ NMR $\left(101 \mathrm{MHz}, \mathrm{CDCl}_{3}\right)$

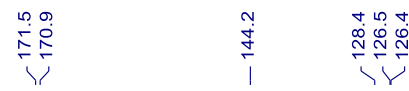

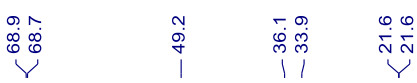

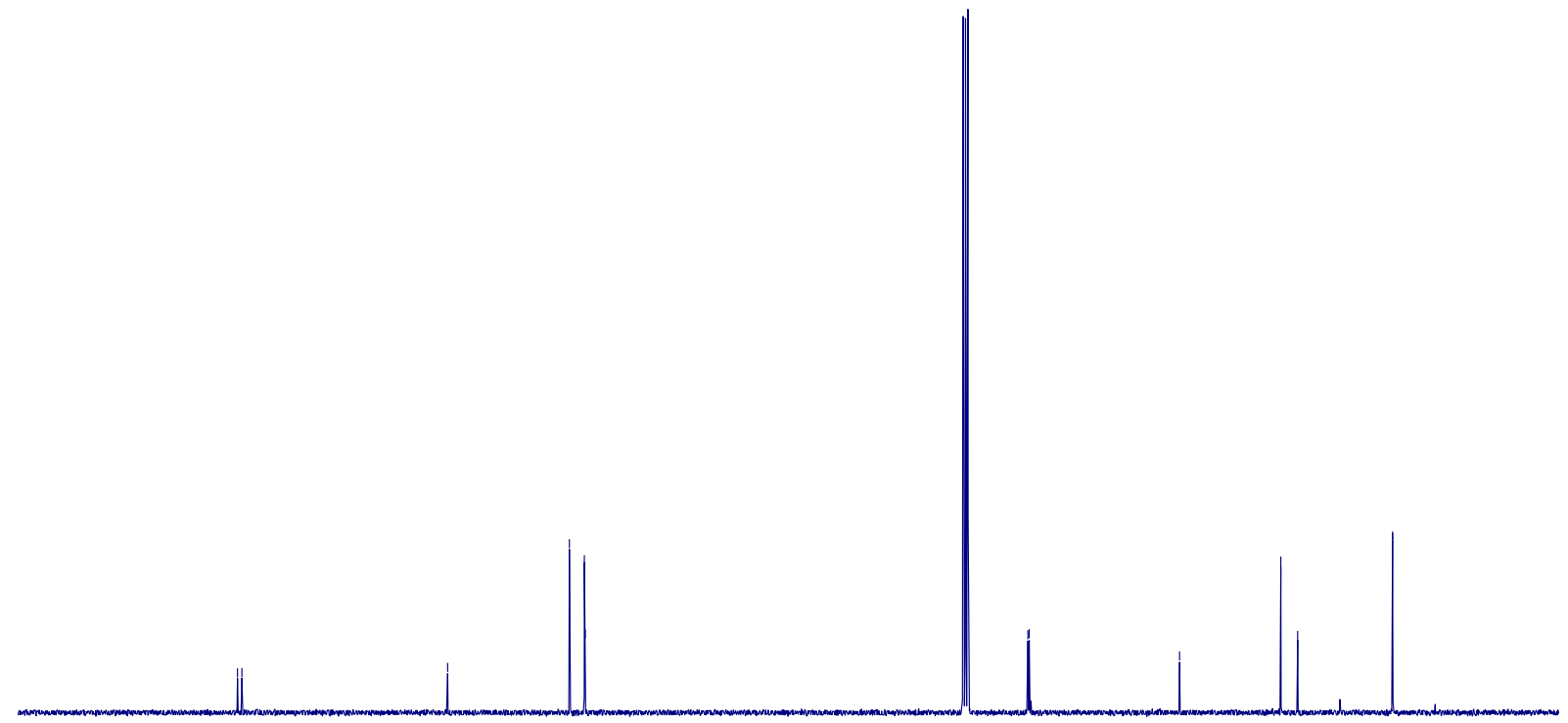

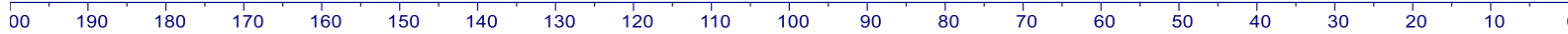


$63-{ }^{1} \mathrm{H}$ NMR (500 MHz, $\mathrm{CDCl}_{3}$ )

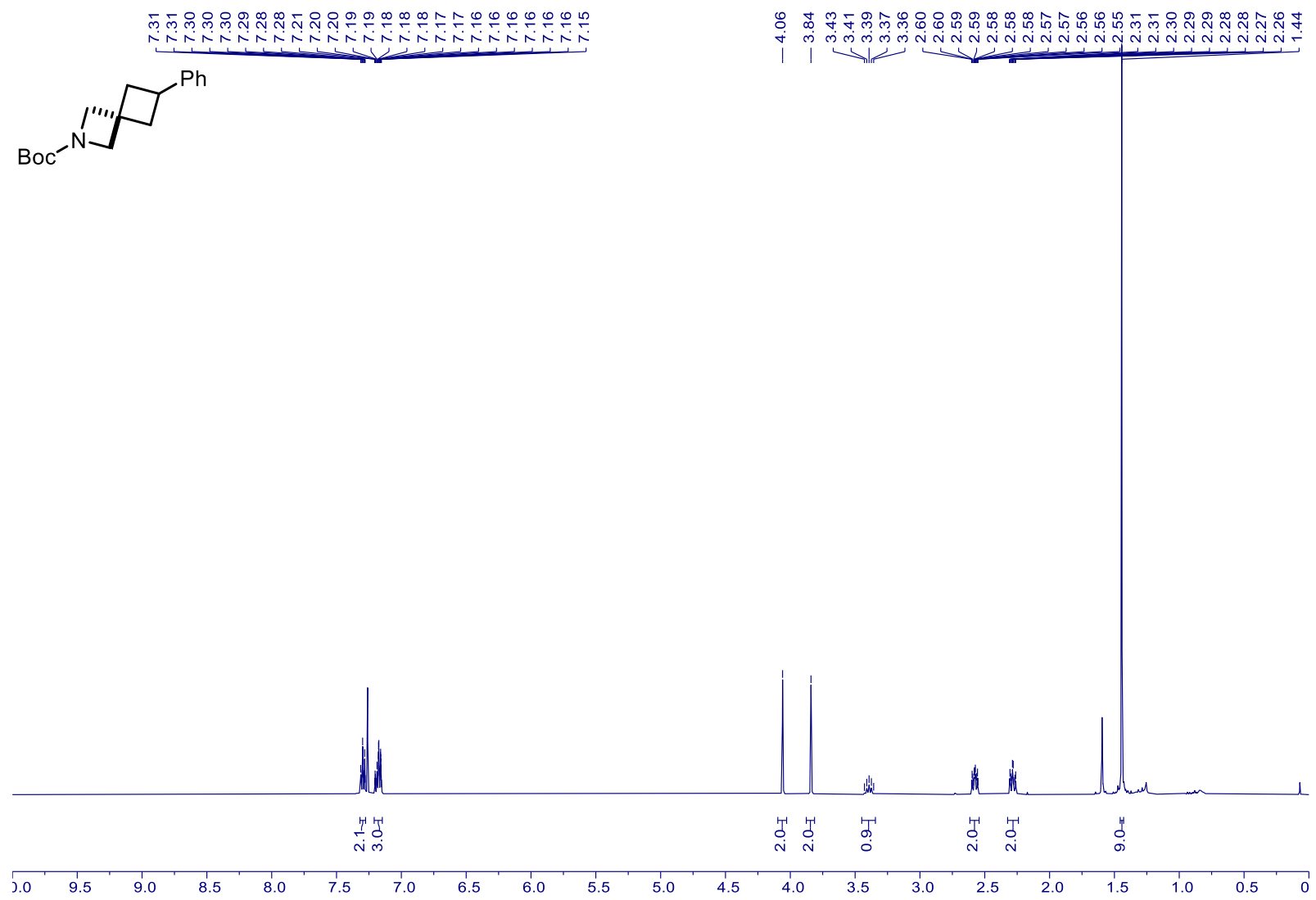

63 $-{ }^{13} \mathrm{C} \mathrm{NMR}\left(126 \mathrm{MHz}, \mathrm{CDCl}_{3}\right)$

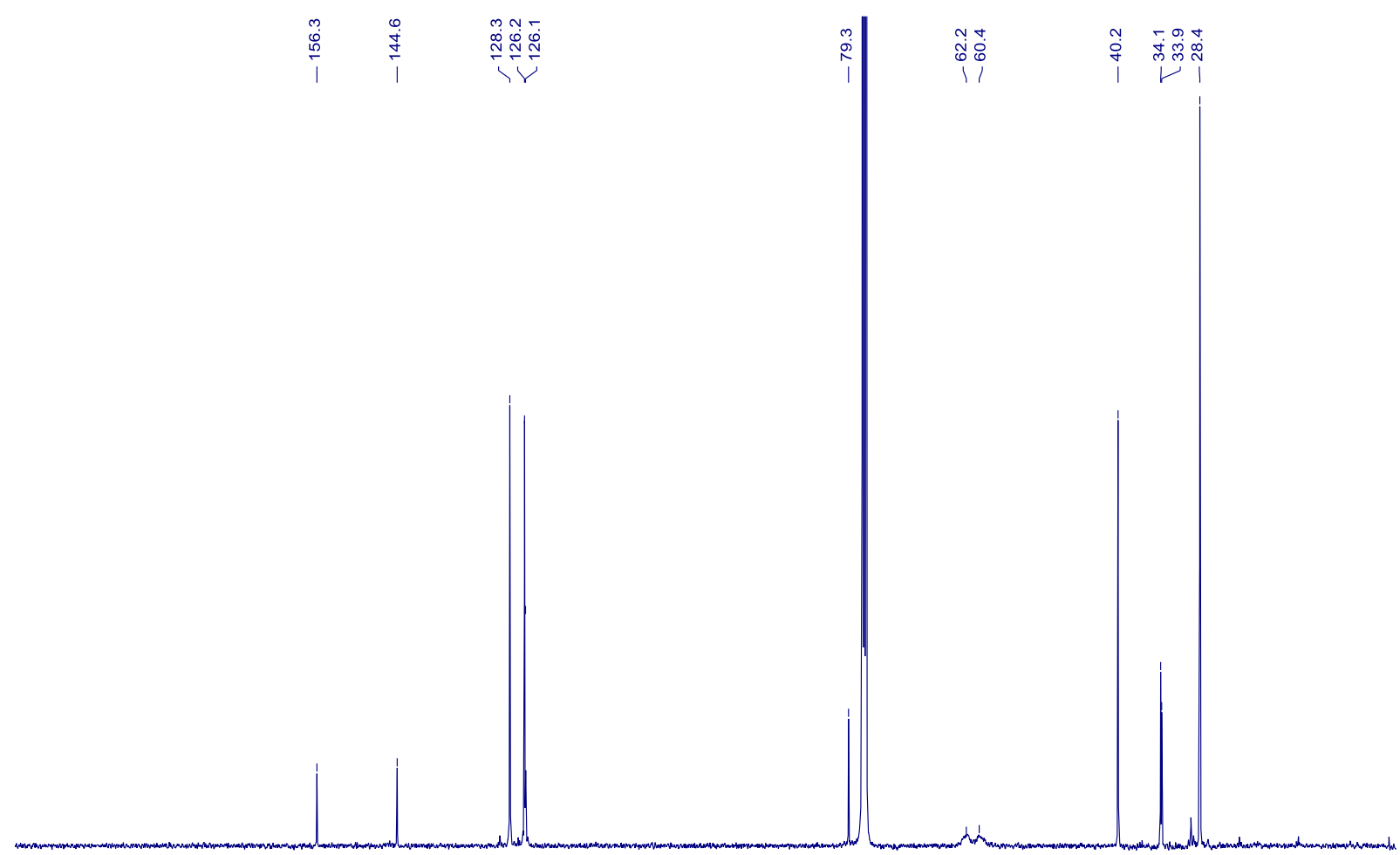

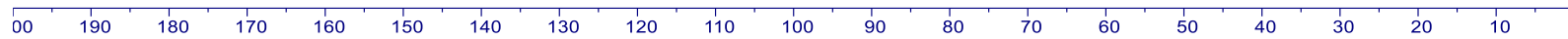


$64-{ }^{1} \mathrm{H}$ NMR $\left(400 \mathrm{MHz}, \mathrm{CDCl}_{3}\right)$

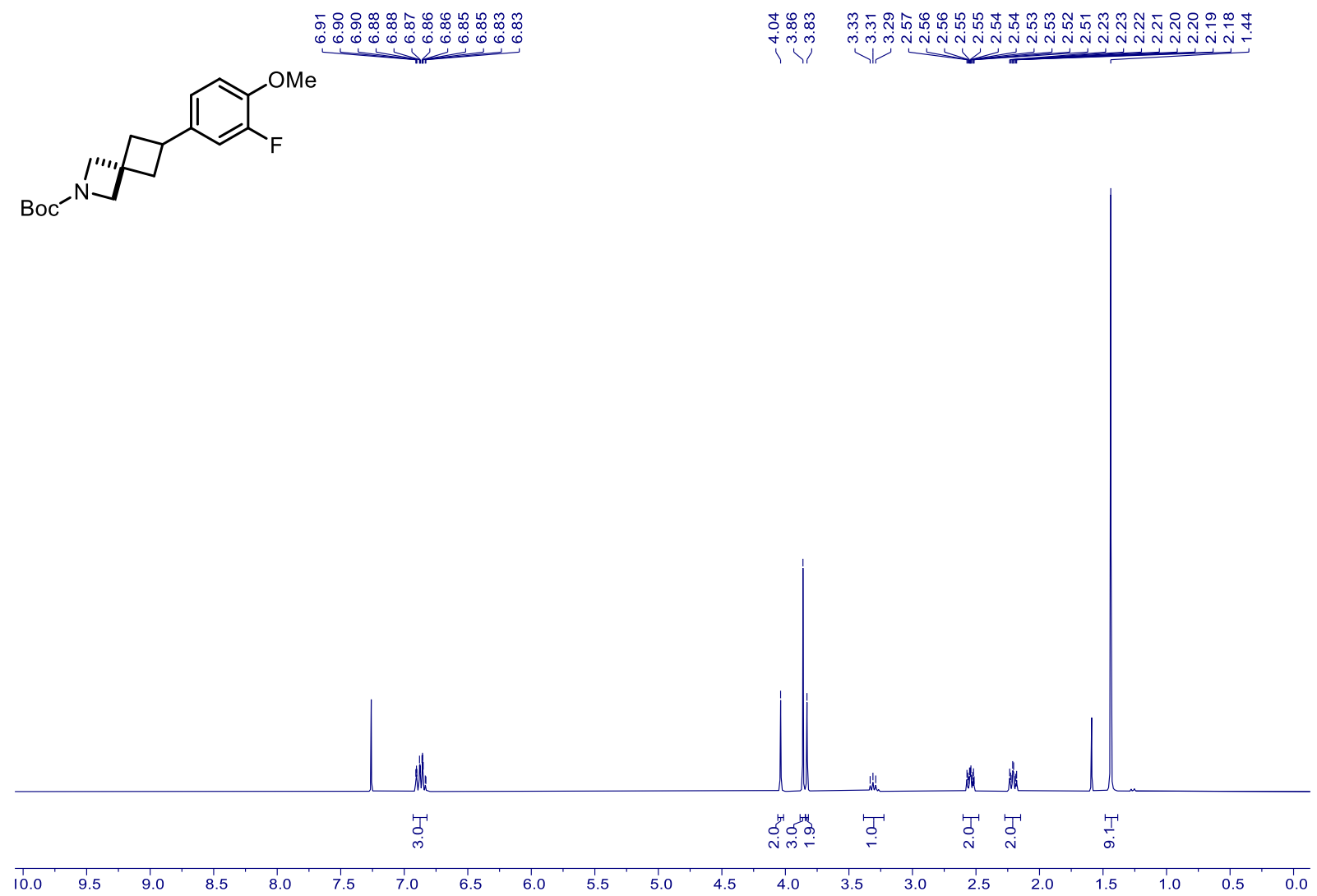

$64-{ }^{13} \mathrm{C}$ NMR (101 MHz, $\left.\mathrm{CDCl}_{3}\right)$

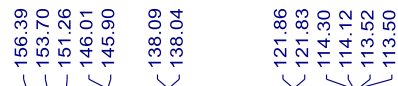

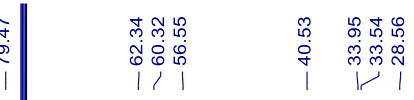

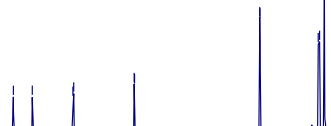

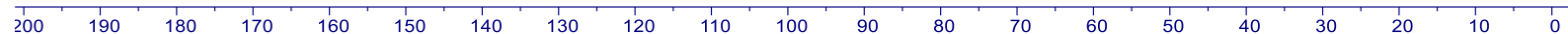


$64-{ }^{19} \mathrm{~F}$ NMR $\left(376 \mathrm{MHz}, \mathrm{CDCl}_{3}\right)$

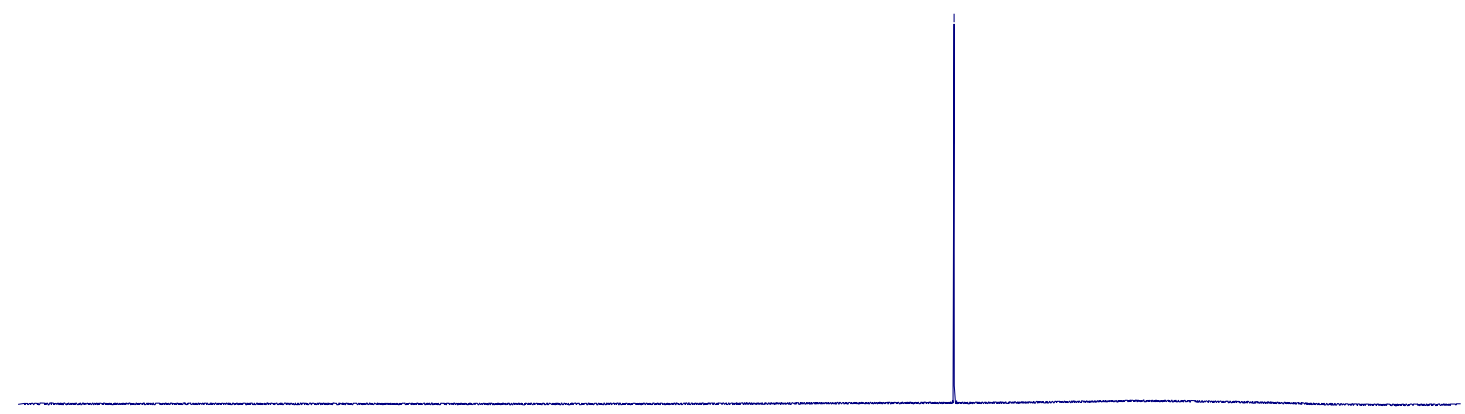

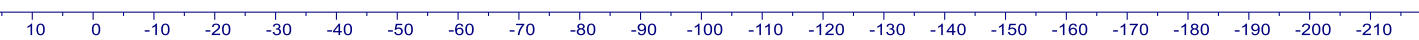


$65-{ }^{1} \mathrm{H}$ NMR $\left(500 \mathrm{MHz}, \mathrm{CDCl}_{3}\right)$

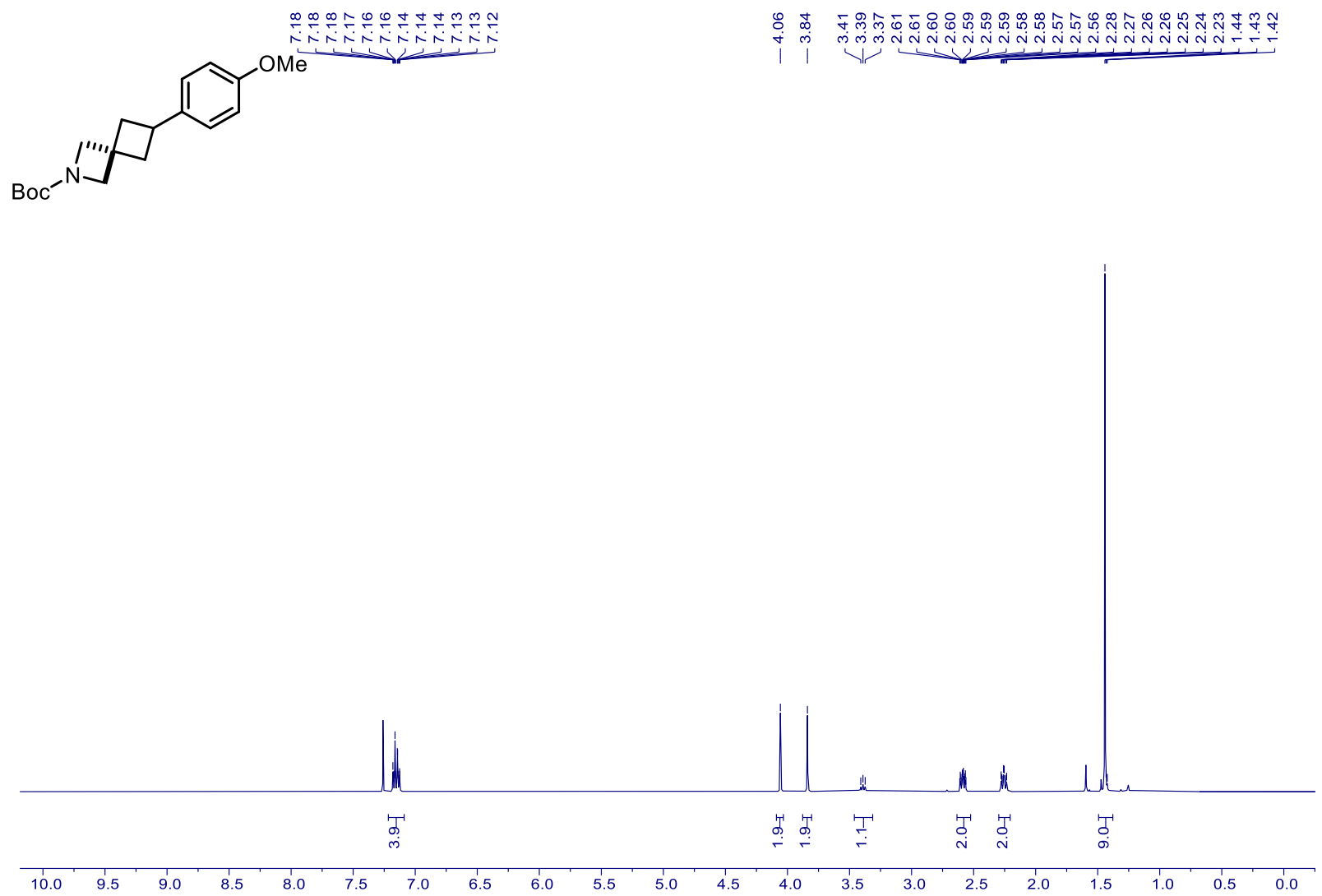

$65-{ }^{13} \mathrm{C}$ NMR $\left(126 \mathrm{MHz}, \mathrm{CDCl}_{3}\right)$

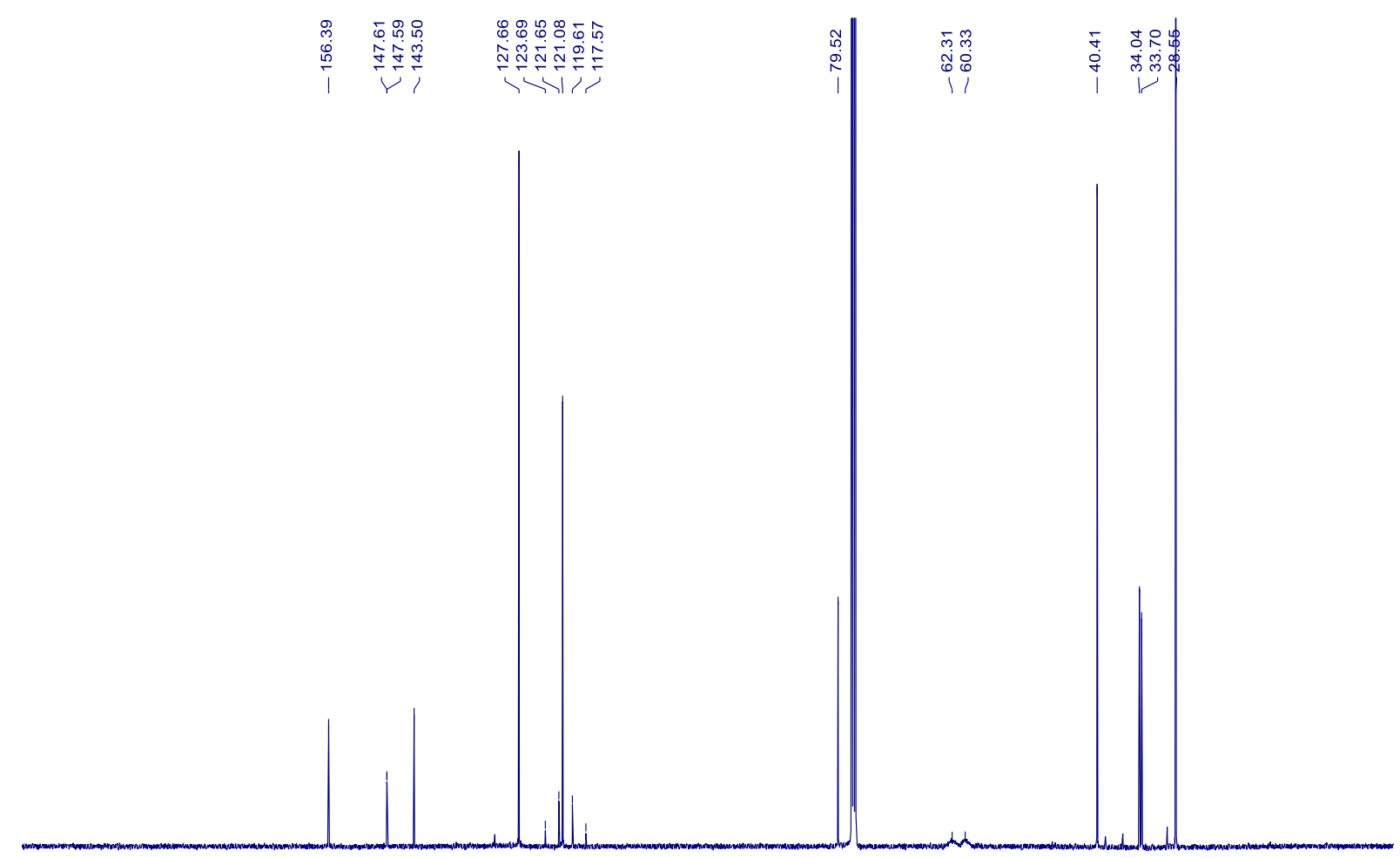

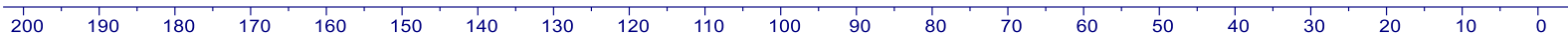


$65-{ }^{19} \mathrm{~F}$ NMR $\left(376 \mathrm{MHz}, \mathrm{CDCl}_{3}\right)$

占

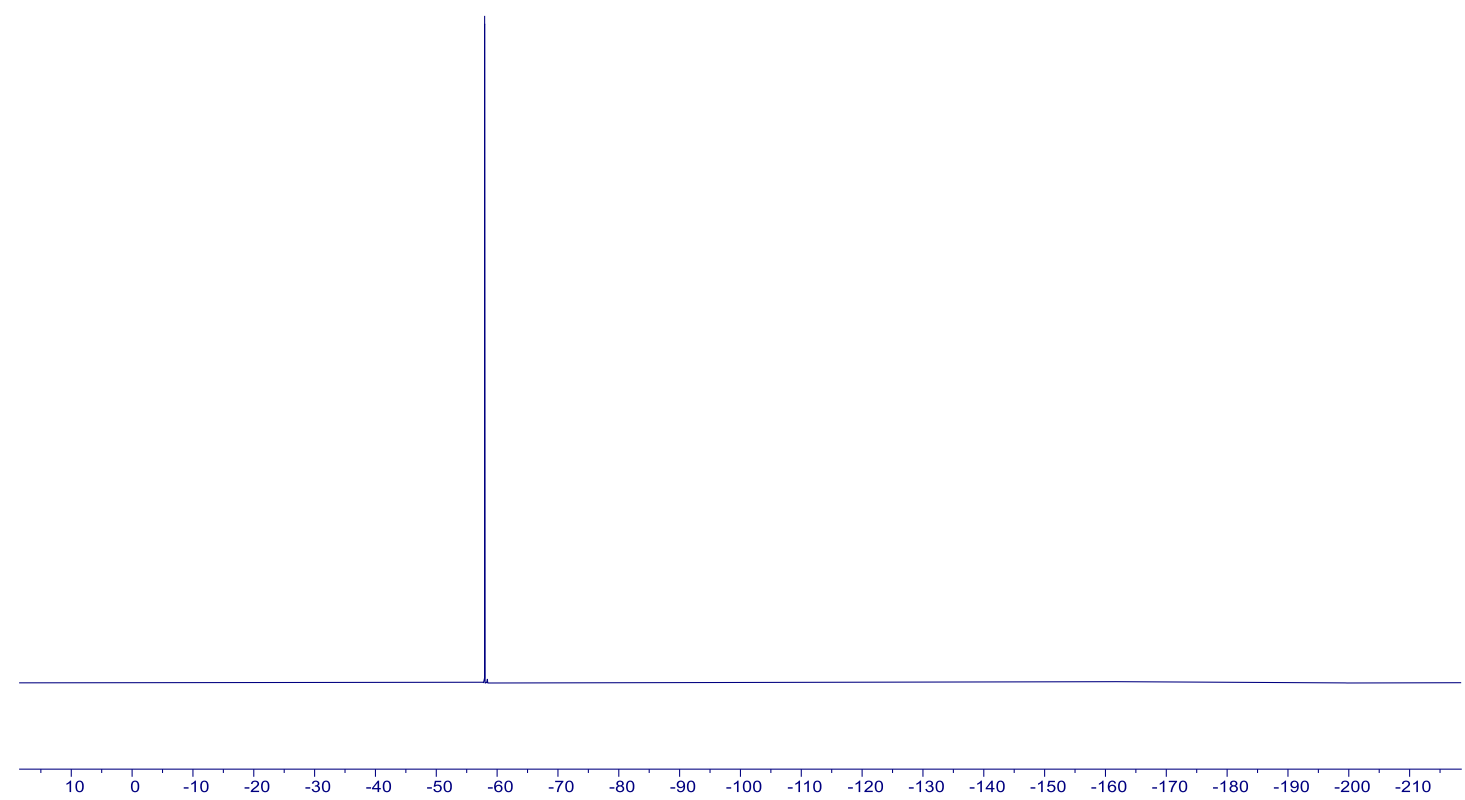


$68-{ }^{1} \mathrm{H}$ NMR $\left(400 \mathrm{MHz}, \mathrm{CDCl}_{3}\right)$

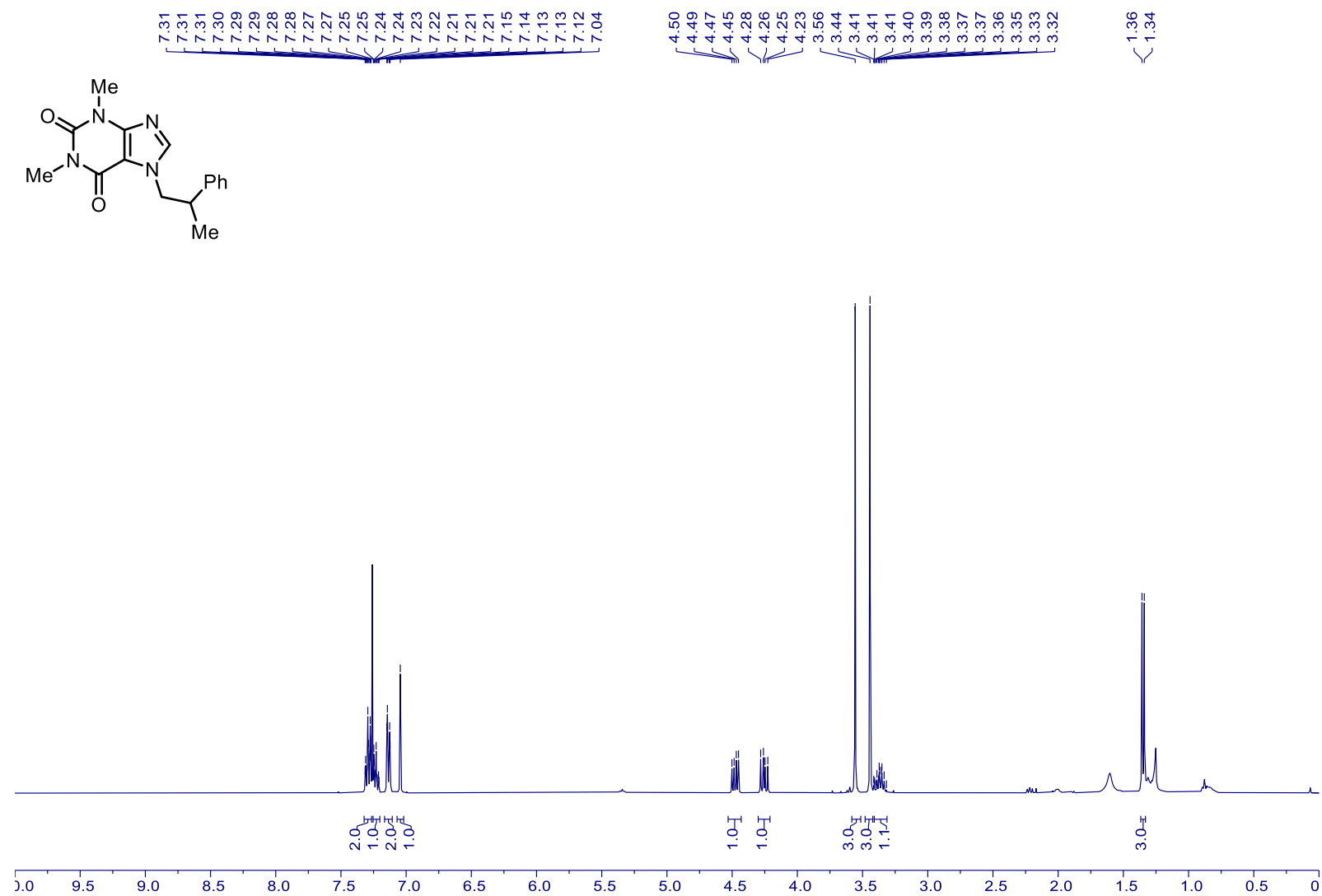

$68-{ }^{13} \mathrm{C}$ NMR $\left(101 \mathrm{MHz}, \mathrm{CDCl}_{3}\right)$

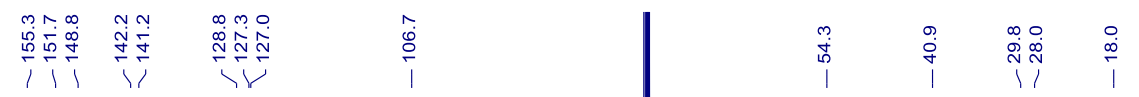

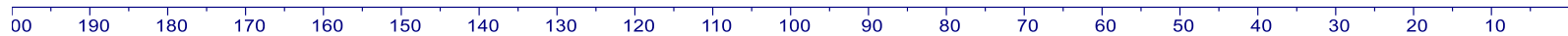


$69-{ }^{1} \mathrm{H}$ NMR (400 MHz, $\left.\mathrm{CDCl}_{3}\right)$<smiles>Cn1cc(C(Cn2cnc3c2c(=O)n(C)c(=O)n3C)C[N+](=O)[O-])cn1</smiles>

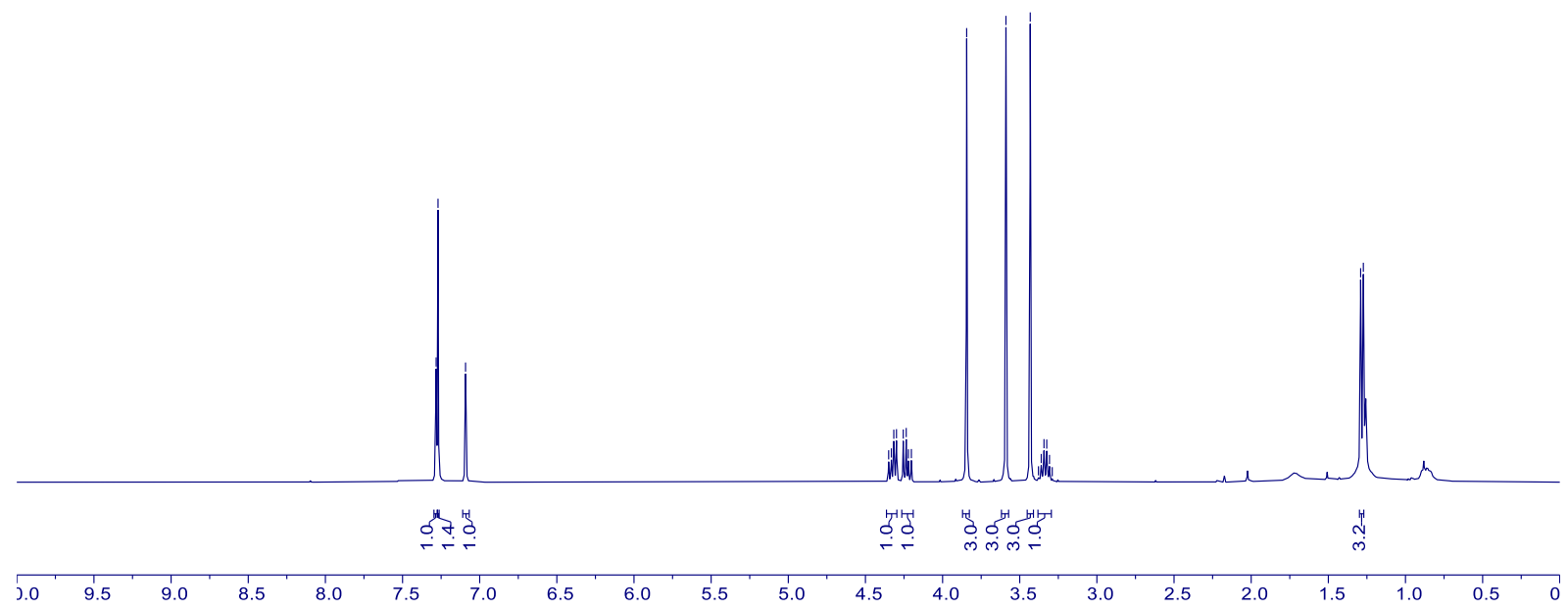

69 $-{ }^{13} \mathrm{C} \mathrm{NMR}\left(101 \mathrm{MHz}, \mathrm{CDCl}_{3}\right)$

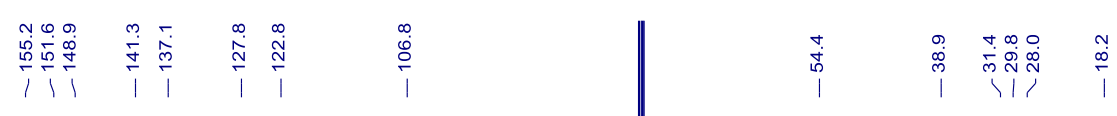


70 - ${ }^{1} \mathrm{H}$ NMR (400 MHz, $\left.\mathrm{CDCl}_{3}\right)$

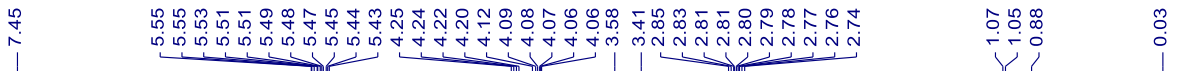<smiles>CCC(C)Cn1cnc2c1c(=O)n(C)c(=O)n2C</smiles>

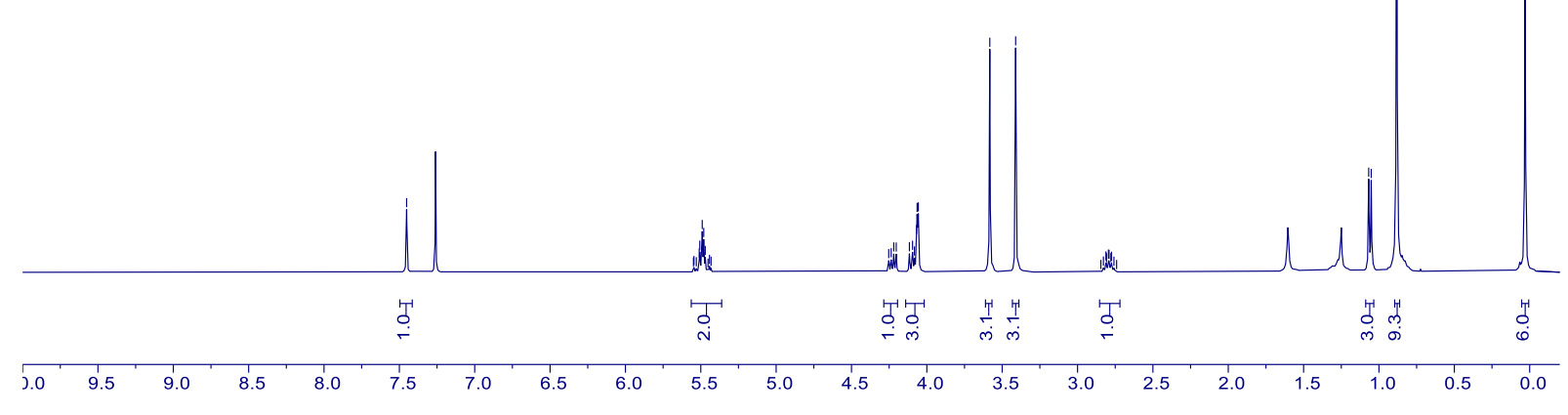

$70-{ }^{13} \mathrm{C}$ NMR (101 MHz, $\left.\mathrm{CDCl}_{3}\right)$

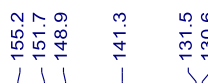

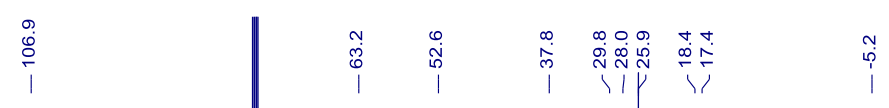

00 \begin{tabular}{llll|}
1 & 1 & 1
\end{tabular} 


\section{Reference}

1. Cheung, C. W.; Ren, P.; Hu, X., Mild and Phosphine-Free Iron-Catalyzed CrossCoupling of Nonactivated Secondary Alkyl Halides with Alkynyl Grignard Reagents. Org. Lett. 2014, 16 (9), 2566-2569.

2. Lutter, F. H.; Grokenberger, L.; Benz, M.; Knochel, P., Cobalt-Catalyzed Csp3-Csp3 Cross-Coupling of Functionalized Alkylzinc Reagents with Alkyl Iodides. Org. Lett. 2020, 22 (8), 3028-3032.

3. Matier, C. D.; Schwaben, J.; Peters, J. C.; Fu, G. C., Copper-Catalyzed Alkylation of Aliphatic Amines Induced by Visible Light. J. Am. Chem. Soc. 2017, 139 (49), 17707-17710.

4. Tissot, M.; Body, N.; Petit, S.; Claessens, J.; Genicot, C.; Pasau, P., Synthesis of Electron-Deficient Heteroaromatic 1,3-Substituted Cyclobutyls via Zinc Insertion/Negishi Coupling Sequence under Batch and Automated Flow Conditions. Org. Lett. 2018, 20 (24), 8022-8025.

5. Górski, B.; Barthelemy, A.-L.; Douglas, J. J.; Juliá, F.; Leonori, D., Copper-catalysed amination of alkyl iodides enabled by halogen-atom transfer. Nat. Catal. 2021, 4 (7), 623-630. 6. Wang, G.-Z.; Jiang, J.; Bu, X.-S.; Dai, J.-J.; Xu, J.; Fu, Y.; Xu, H.-J., CopperCatalyzed Cross-Coupling Reaction of Allyl Boron Ester with $1 \% 2^{\circ} / 3^{\circ}$-Halogenated Alkanes. Org. Lett. 2015, 17 (15), 3682-3685.

7. Chen, K.-Q.; Wang, Z.-X.; Chen, X.-Y., Photochemical Decarboxylative C(sp3)-X Coupling Facilitated by Weak Interaction of N-Heterocyclic Carbene. Org. Lett. 2020, 22 (20), 8059-8064.

8. Chen, Y.; Ma, G.; Gong, H., Copper-Catalyzed Reductive Trifluoromethylation of Alkyl Iodides with Togni's Reagent. Org. Lett. 2018, 20 (15), 4677-4680.

9. Caiger, L.; Sinton, C.; Constantin, T.; Douglas, J. J.; Sheikh, N. S.; Juliá, F.; Leonori, D., Radical hydroxymethylation of alkyl iodides using formaldehyde as a C1 synthon. Chem. Sci. 2021, 12 (31), 10448-10454.

10. Rao, D. S.; Reddy, T. R.; Babachary, K.; Kashyap, S., Regioselective vicinal functionalization of unactivated alkenes with sulfonium iodate(i) reagents under metal-free conditions. Org. Biomol. Chem. 2016, 14 (31), 7529-7543.

11. Kyne, S. H.; Clémancey, M.; Blondin, G.; Derat, E.; Fensterbank, L.; Jutand, A.; Lefèvre, G.; Ollivier, C., Elucidating Dramatic Ligand Effects on SET Processes: Iron Hydride versus Iron Borohydride Catalyzed Reductive Radical Cyclization of Unsaturated Organic Halides. Organometallics 2018, 37 (5), 761-771.

12. Yadav, J. S.; Reddy, B. V. S.; Krishna, V. H.; Swamy, T.; Kumar, G. K. N., Iodinepromoted Prins-cyclization of ketones - A facile synthesis of spirocyclic-4-iodotetrahydropyrans and 5,6-dihydro-2H-pyrans. Can. J. Chem. 2007, 85 (6), 412-415.

13. K., S. A.; Somasekhar, B.; Kiran, I.; Paramartha, G., Axial-selectivity in Prins Cyclization Reaction: Synthesis of 4-Iodotetrahydropyrans. Chem. Lett. 2011, 40 (10), 11761178.

14. Sakamoto, R.; Sakurai, S.; Maruoka, K., Alkylsilyl Peroxides as Alkylating Agents in the Copper-Catalyzed Selective Mono-N-Alkylation of Primary Amides and Arylamines. Chem. Eur. J. 2017, 23 (38), 9030-9033.

15. Parmar, D.; Henkel, L.; Dib, J.; Rueping, M., Iron catalysed cross-couplings of azetidines - application to the formal synthesis of a pharmacologically active molecule. Chem. Commun. 2015, 51 (11), 2111-2113.

16. Roagna, G.; Ascough, D. M. H.; Ibba, F.; Vicini, A. C.; Fontana, A.; Christensen, K. E.; Peschiulli, A.; Oehlrich, D.; Misale, A.; Trabanco, A. A.; Paton, R. S.; Pupo, G.; Gouverneur, V., Hydrogen Bonding Phase-Transfer Catalysis with Ionic Reactants: Enantioselective Synthesis of $\gamma$-Fluoroamines. J. Am. Chem. Soc. 2020, 142 (33), 14045-14051. 17. Molander, G. A.; Traister, K. M.; O’Neill, B. T., Reductive Cross-Coupling of 
Nonaromatic, Heterocyclic Bromides with Aryl and Heteroaryl Bromides. J. Org. Chem. 2014, 79 (12), 5771-5780.

18. Liu, Z.; Luan, N.; Shen, L.; Li, J.; Zou, D.; Wu, Y.; Wu, Y., Palladium-Catalyzed Hiyama Cross-Couplings of Arylsilanes with 3-Iodoazetidine: Synthesis of 3-Arylazetidines. $J$. Org. Chem. 2019, 84 (19), 12358-12365.

19. Bhonde, V. R.; O'Neill, B. T.; Buchwald, S. L., An Improved System for the Aqueous Lipshutz-Negishi Cross-Coupling of Alkyl Halides with Aryl Electrophiles. Angew. Chem., Int. Ed. 2016, 55 (5), 1849-1853.

20. Duncton, M. A. J.; Estiarte, M. A.; Tan, D.; Kaub, C.; O’Mahony, D. J. R.; Johnson, R. J.; Cox, M.; Edwards, W. T.; Wan, M.; Kincaid, J.; Kelly, M. G., Preparation of Aryloxetanes and Arylazetidines by Use of an Alkyl-Aryl Suzuki Coupling. Org. Lett. 2008, 10 (15), 3259-3262.

21. Barré, B.; Gonnard, L.; Campagne, R.; Reymond, S.; Marin, J.; Ciapetti, P.; Brellier, M.; Guérinot, A.; Cossy, J., Iron- and Cobalt-Catalyzed Arylation of Azetidines, Pyrrolidines, and Piperidines with Grignard Reagents. Org. Lett. 2014, 16 (23), 6160-6163.

22. Dequirez, G.; Bourotte, M.; Porras de Francisco, E.; Remuiñan Blanco, M. J.; Déprez, B.; Willand, N., Microwave-Assisted Suzuki-Miyaura Cross Coupling using Nickel as Catalyst to Rapidly Access to 3-Arylazetidine. ChemistrySelect 2017, 2 (28), 8841-8846.

23. Yamaguchi, T. I., K.; Hirano, T.; Shimabara, R.; Kawakubo. Y.; Sato, M.; Yamashita, J.; Yazaki, A.; Ueshima, T. Novel Pyridone Carboxylic Acid Derivative or Salt Thereof. U.S. Patent US2020062752. February 27, 2020.

24. Han, S.; Ren, X.; Wu, Q.; Liang, A.; Li, J.; Zou, D.; Wu, Y.; Wu, Y., PalladiumCatalyzed Decarboxylative Cross-Couplings of 1-Boc-3-iodoazetidine: Regioselective Access to 2-Alkynylazetidines, 3-Alkynylazetidines and 3-Vinylazetidines. Adv. Synth. Catal. 2018, 360 (12), 2308-2312.

25. Di Franco, T.; Epenoy, A.; Hu, X., Synthesis of E-Alkyl Alkenes from Terminal Alkynes via Ni-Catalyzed Cross-Coupling of Alkyl Halides with B-Alkenyl-9borabicyclo[3.3.1]nonanes. Org. Lett. 2015, 17 (19), 4910-4913.

26. De Francisco, E. P. R.-B., M. J.; Bourotte, M.; Deprez, D.; Dequirez, G.; Willand, N. Novel Compounds. US Patent US2020170997. June 4, 2020.

27. Qiu, Z.; Zhu, M.; Zheng, L.; Li, J.; Zou, D.; Wu, Y.; Wu, Y., Regioselective $\alpha-$ benzylation of 3-iodoazetidine via Suzuki cross-coupling. Tetrahedron Lett. 2019, 60 (19), 1321-1324.

28. Campbell, P. S.; Jamieson, C.; Simpson, I.; Watson, A. J. B., Practical synthesis of pharmaceutically relevant molecules enriched in sp3 character. Chem. Commun. 2018, 54 (1), 46-49.

29. Li, Z.; Sun, W.; Wang, X.; Li, L.; Zhang, Y.; Li, C., Electrochemically Enabled, Nickel-Catalyzed Dehydroxylative Cross-Coupling of Alcohols with Aryl Halides. J. Am. Chem. Soc. 2021, 143 (9), 3536-3543.

30. Liu, X.-G.; Zhou, C.-J.; Lin, E.; Han, X.-L.; Zhang, S.-S.; Li, Q.; Wang, H., Decarboxylative Negishi Coupling of Redox-Active Aliphatic Esters by Cobalt Catalysis. Angew. Chem., Int. Ed. 2018, 57 (40), 13096-13100.

31. Guo, W.; Laserna, V.; Rintjema, J.; Kleij, A. W., Catalytic One-Pot Oxetane to Carbamate Conversions: Formal Synthesis of Drug Relevant Molecules. Adv. Synth. Catal. 2016, 358 (10), 1602-1607.

32. Masanobu, K.; Akito, I.; Yasunao, K.; Yuji, W.; Haruhiko, T., Study of Reactive Intermediates in Photoinduced Electron Transfer Anti-Markovnikov Addition of Methanol to 1-Phenylcycloalkenes. Bull. Chem. Soc. Jpn. 1999, 72 (5), 1049-1055.

33. Chatterjee, A.; König, B., Birch-Type Photoreduction of Arenes and Heteroarenes by Sensitized Electron Transfer. Angew. Chem., Int. Ed. 2019, 58 (40), 14289-14294. 
34. Carter, T. S.; Guiet, L.; Frank, D. J.; West, J.; Thomas, S. P., Iron-Catalysed Reduction of Olefins using a Borohydride Reagent. Adv. Synth. Catal. 2013, 355 (5), 880-884.

35. Reddy, U. C.; Bondalapati, S.; Saikia, A. K., Stereoselective One-Pot, ThreeComponent Synthesis of 4-Aryltetrahydropyran via Prins-Friedel-Crafts Reaction. J. Org. Chem. 2009, 74 (6), 2605-2608.

36. Goetzke, F. W.; Hell, A. M. L.; van Dijk, L.; Fletcher, S. P., A catalytic asymmetric cross-coupling approach to the synthesis of cyclobutanes. Nat. Chem. 2021, 13 (9), 880-886.

37. Kuang, Y.; Wang, X.; Anthony, D.; Diao, T., Ni-catalyzed two-component reductive dicarbofunctionalization of alkenes via radical cyclization. Chem. Commun. 2018, 54 (20), 2558-2561.

38. Kim, J. G.; Son, Y. H.; Seo, J. W.; Kang, E. J., Iron-Catalyzed Tandem Cyclization and Cross-Coupling Reactions of Iodoalkanes and Aryl Grignard Reagents. Eur. J. Org. Chem. 2015, 2015 (8), 1781-1789.

39. Aranzaes, J. R.; Daniel, M.-C.; Astruc, D., Metallocenes as references for the determination of redox potentials by cyclic voltammetry - Permethylated iron and cobalt sandwich complexes, inhibition by polyamine dendrimers, and the role of hydroxy-containing ferrocenes. Can. J. Chem. 2006, 84 (2), 288-299.

40. Roth, H. G.; Romero, N. A.; Nicewicz, D. A., Experimental and Calculated Electrochemical Potentials of Common Organic Molecules for Applications to Single-Electron Redox Chemistry. Synlett 2016, 27 (05), 714-723. 